\title{
Full-coverage native RNA sequencing of HIV-1 viruses
}

Alejandro R. Gener ${ }^{1,2,3,4}$, Jason T. Kimata ${ }^{5}$

${ }^{1}$ Integrative Molecular and Biomedical Sciences Program, Graduate School of Biomedical

Sciences, Baylor College of Medicine, Houston, Texas, USA

${ }^{2}$ Margaret M. and Albert B. Alkek Department of Medicine, Nephrology, Baylor College of Medicine, Houston, Texas, USA

${ }^{3}$ Department of Genetics, MD Anderson Cancer Center, Houston, Texas, USA

${ }^{4}$ School of Medicine, Universidad Central del Caribe, Bayamón, Puerto Rico, USA

${ }^{5}$ Department of Molecular Virology and Microbiology, Baylor College of Medicine, Houston, Texas, USA

\section{Corresponding author}

Alejandro R. Gener (ARG): gener@bcm.edu ; itspronouncedhenner@gmail.com

\section{Conflict(s) of interest:}

ARG received travel bursaries from Oxford Nanopore Technologies (ONT), Oxford, UK.

The present work was completed independently of ONT. JTK declares no conflict of interest.

Key words: HIV-1, nanopore, native RNA sequencing, HIV sequence variability, retrovirus, viral heterogeneity, Context Dependency Variant Class

Funding: This work was funded in part by institutional support from Baylor College of Medicine; private funding by East Coast Oils, Inc., Jacksonville, Florida, and ARG's own private funding, including Student Genomics (manuscripts in prep). ARG also received the 
bioRxiv preprint doi: https://doi.org/10.1101/845610; this version posted November 19, 2019. The copyright holder for this preprint (which was not certified by peer review) is the author/funder. All rights reserved. No reuse allowed without permission.

PFLAG of Jacksonville scholarship for multiple years. The work was also supported in part

by NIH grant AI116167 to JTK. 


\section{ABSTRACT}

Objective: To evaluate native RNA sequencing for sequencing HIV-1 viral genomes.

Methods: Fifteen HIV-1 strains were processed with Direct RNA Sequencing (SQKRNA002) library kits and sequenced on MinION Mk1B devices with RevD flow cells (Oxford Nanopore Technologies (ONT), Oxford, UK). Raw reads were converted to FASTQ, aligned to reference sequences, and assembled into contigs. Multi-sequence alignments of the contigs were generated and used for cladistics analysis.

Results: We sequenced full-length HIV-1 from the transcriptional start site to 3' LTR (100\% virion genome) in 3 out of 15 isolates (89.6, NLAD8, AD17), achieving majority coverage (defined as $>50 \%$ ) in another 7 out of 15 isolates. Inspection of NLAD8 sequence alignments revealed splicing or deletion signatures. Despite the strong 3' bias, read coverage was sufficient to evaluate single-nucleotide variants (SNVs), insertions and deletions in 9 isolates, and to assemble HIV-1 genomes directly from viral RNA, achieving a maximum of $94 \%$ assembly coverage for NLAD8. Phylogenetic relationships were maintained at the level of contigs, as well as individual reads.

Conclusions: ONT native RNA sequencing performed as expected, covering full-length HIV-1 RNA without PCR or cDNA sequencing. Native single-molecule RNA sequencing supported previous models of HIV-1 replication, and samples exhibited strain-specific transcriptional signals. We propose Context Dependency Variant Classification to describe variants occurring in information-dense regions of HIV. These data provide rich resources for emerging RNA modification detection schemes. Future work will expand HIV-1 transcript profiling to infection models and clinical samples. 


\section{INTRODUCTION}

Human immunodeficiency virus type 1 (HIV-1) strains have high sequence

variability due to their highly error-prone replication. Lack of full-length sequencing data has limited our understanding of HIV biology. The closest we have come to being able to observe the information contained in the HIV-1 viral genome directly has been to stitch together short read data into quasispecies [1], which are neither real nor direct observations of these viruses. Most approaches to sequencing the HIV-1 viral genome have used some variant of reverse transcription to make double-stranded cDNA, usually followed by PCR amplification, and finally cDNA sequencing (classic RNA-seq) [2].

Recently, we used long read PCR + DNA sequencing of synthetic plasmid-bound provirus to observe the entire HIV-1 DNA genome for the first time [3]. In addition to covering the HIV-1 reference sequence HXB2 deeply and evenly, and recovering at least one full-length read, we were able to observe 20 SNVs, half of which were in repetitive Long Terminal Repeat (LTR) regions. However, DNA-based sequencing methods cannot differentiate between reads from infectious virion RNA, integrated proviral DNA, and non-integrated forms [4]. Direct sequencing of the entire HIV-1 viral RNA genome with one full-length read of native virion-associated RNA has yet to be described. A barrier to full-coverage HIV-1 reads has been limitations in lengths of extracted nucleic acids from samples (Gener, unpublished). Additionally, HIV-1 exhibits complicated splicing behavior which may be virus- [5] and host cell-dependent [6]. The observation of alternative splicing of viral mRNAs [5] is coverage-dependent, often forcing investigators into using PCR to amplify signal at the expense of per-base accuracy. PCR also biases the signal that can be observed. The Oxford Nanopore Technologies (ONT) nanopore sequencers are the only commercially available platforms currently able to directly sequence RNA in its native 
form without the need for CDNA sequencing or PCR [7], and have been used to sequence other important RNA viruses like influenza A virus [8]. Here, we used MinION Mk1B devices to sequence 15 strains of full-length infectious HIV-1, with the goal of evaluating the technology for future applications. 


\section{METHODS}

\section{HIV-1 viruses}

To generate infectious virus from plasmid clones of HIV-1 NL4-3, NLAD8, RHPA, and AD17, we transiently transfected 293T cells using ExtremeGENE 9 according to the manufacturer's protocol (Roche). After 48 hours, supernatants were collected from transfected cultures of cells, passed through $0.45 \mu \mathrm{m}$-pore-size syringe filters, aliquoted, and saved at $-80^{\circ} \mathrm{C}$. The amount of infectious virus was determined by limiting dilution on TZMbl cells using a luciferase reporter assay as we have described [9].

Other HIV-1 strains used in this study were derived from infectious virus obtained from the NIH AIDS Reagent and Reference Program (89.6, SF162, 97USNG30, MN, ADA, 92UG038, 92UG029, ELI, JR-FL, and BaL) or were generously provided by M.R. Ferguson and W.A. O'Brien (HIV1-SX) of the University of Texas Medical Branch, Galveston, TX. In short, peripheral blood mononuclear cells (PBMCs) were isolated from leukopacks of anonymous blood donors purchased from the Gulf Coast Blood Center (Houston, TX) using Lymphocyte Separation Medium (Sigma-Aldrich). Cultures of PBMCs were stimulated with phytohemagglutinin PHA-P and IL-2 $(50 \mathrm{U} / \mathrm{ml})$ in RPMI1640 complete (supplemented with $10 \%$ heat-inactivated fetal bovine serum, $2 \mathrm{mM}$ L-glutamine, and 100 $\mathrm{U} / \mathrm{ml}$ penicillin/ $100 \mu \mathrm{g} / \mathrm{ml}$ streptomycin) for 2 days prior to inoculation with HIV-1. After infection, PBMCs were cultured for an additional 10-15 days in RPMI complete with 50 $\mathrm{U} / \mathrm{ml} \mathrm{IL-2}$. Virus production was confirmed by testing supernatants for HIV-1 p24 by ELISA (Advanced Bioscience Laboratories). Supernatants were collected from infected cultures of PBMCs, passed through $0.45 \mu \mathrm{m}$-pore-size syringe filters, aliquoted and saved at $-80{ }^{\circ} \mathrm{C}$. The amount of infectious virus was determined by limiting dilution titration of the virus 
stocks on PHA-stimulated PBMCs and assay for infectious virus production after 10-14 days of infection by HIV-1 p24 ELISA.

\section{Viral RNA isolation}

RNA was extracted using the MagMAX-96 viral RNA isolation kit (Applied Biosystems) with a Kingfisher Flex Purification System (ThermoFisher) described by the manufacturer with the following modifications: $200 \mu$ l of viral lysate was added to $200 \mu l$ of freshly prepared Lysis/Binding Solution and allowed to incubate for at least 15 minutes (no more than 1 hour). Each lysate sample was loaded into a well of a 96-well deep well Kingfisher plate and processed following kit instructions. Each sample was eluted into 50 ul of Elution Buffer in duplicate into individual DNA LoBind $1.5 \mathrm{ml}$ microcentrifuge tubes (Eppendorf) for storage at $80^{\circ} \mathrm{C}$ until use in sequencing.

\section{Long-read native RNA sequencing}

Extracted RNA was sequenced across three MinION Mk1B sequencers (Oxford Nanopore Technologies (ONT), Oxford, UK). Briefly, 9 ul of extracted viral RNA eluate was carried over and processed with Direct RNA kit SQK-RNA002 following ONT protocol DRS_9080_v2_revF_22Nov2018, Last updated: 19/07/2019. This protocol recommended 1 round of cDNA "first-strand" synthesis for the purpose of breaking up RNA 20 structure, however these cDNA strands are ultimately not sequenced. The reverse transcriptase kit used: SuperScript IV First Strand Synthesis System (Invitrogen). The ligase used: T4 DNA ligase (NEB). Libraries were loaded onto one of 5 MinION RevD flow cells, pore version R9.4.1. and sequencing runs approximating 6 to 11 hours were completed with MinKNOW (version 3.5.5) on three laptop computers.

Runs were stopped manually in MinKNOW based on empirical assessment of HIV1 sequence coverage while Live Basecalling (ONT) was enabled. Between runs, flow cells 
were washed with Flow Cell Wash Kit (EXP-WSH003) (Version:

WFC_9088_v1_revB_18Sep2019). Flow cells exhibiting increasing failed reads, or abrupt changes in membrane potential (bottoming out at $-250 \mathrm{mV}$ ) were replaced with new flow cells. Flow cell resting potential was set at $-180 \mathrm{mV}$ at the beginning of each run, and MinKNOW was used to adjust voltage automatically at mux scans every 30 minutes during the runs.

Raw data was basecalled (converted from FAST5 to FASTQ format) with Guppy high-accuracy model version 3.3.0, on a compute cluster. Mapped basecalled reads were fed into Canu version 1.8 implemented on a cluster [10]. The parameter genomeSize in Canu was set to be $1 \mathrm{~Kb}$, and stopOnLowCoverage was set to 10 reads. SAMTOOLS Flagstat [11] and QualiMap [12] were used for mapping stats from BAM files from minimap2 [13] implemented in Galaxy (usegalaxy.eu) [14]. SnapGene version 5.0.4 was used to manually annotate contigs from Canu. Select regions were interrogated with multisequence alignments with MAFFT [15] and visualized with Archaeopteryx 0.9921 beta (170712).

After basecalling, read length and mean quality score are reported in a sequencing_symmary.txt file in MinKNOW. For skipped reads (reads not basecalled by timed or forced end of sequencing runs), basecalling in standalone Guppy provides the same sequencing_symmary.txt information. For HIV-1-mapping reads, this information is provided in Figure 1. To pull this information, unique read IDs aligning to AF033819 (with minimap2) were concatenated into a master list and used as follows: egrep -f MASTERLIST.txt sequencing_symmary.txt > HIV-mapping_sequencing_symmary.txt. Data was wrangled in Microsoft Excel for Mac version 16.29.1. Graphing was done with 
GraphPad Prism 8 for macOS version 8.3.0. As samples were not compared directly to each other, additional statistics were not calculated.

Reference sequences

The reference sequence of HIV-1 used in Figure $2 \mathrm{~A}-\mathbf{0}$ was from NCBI, accession number AF033819.3. Supplemental Figure 11 used HXB2 K03455.1. See Table 1 for additional reference accession numbers. The control RNA in SQK-RNA002 is ENO2, and the reference used for mapping was NM_001179305, a partial mRNA from Saccharomyces cerevisiae S288C phosphopyruvate hydratase ENO2 (ENO2), 1314 bp long. 


\section{RESULTS}

\section{Coverage}

HIV-1-mapping reads were recovered in all $(n=15)$ experiments (Figures $\mathbf{1}, \mathbf{2})$.

Three out of 15 strains had full-coverage, defined as read length spanning from the TSS to the 3' LTR and mapped to AF033819.3. These were: $89.6(9,166 / 9,181 \sim 99.8 \%)$, AD17 $(9,164 / 9,181 \sim 99.8 \%)$, and NLAD8 (9,166/9,181 99.8\%). Seven out of 15 strains had majority coverage, defined as $>50 \%$ relative to AF033819.3. These were: 97 USNG30 (6,992/9,181 76.2\%), BaL (8,597/9,181 93.6\%), ELI (6,987/9,181 76.1\%), MN (5,995/9,181 65.3\%), NL4-3 (4,715/9,181 51.4\%), RHPA (4,745/9,181 51.7\%), and HIV1SX $(5,561 / 9,181 \sim 60.6 \%)$. Three out of 15 strains had moderate coverage $<50 \%$ and $>25 \%$ relative to AF033819.3. These were: $92 \mathrm{UG038}(3,678 / 9,181 \sim 40.1 \%)$, JR-FL $(2,814 / 9,181 \sim 30.7 \%)$, and SF162 (2,601/9,181 28.3\%). Two out of 15 strains had lower coverage <25\% relative to AF033819.3. These were: 92 UG029 (2,007/9,181 21.9\%), ADA $\left(1,981 / 9,181^{\sim} 21.6 \%\right)$. In general, samples with higher $\mathrm{TCID}_{50} / \mathrm{ml}$ were more likely to produce more and longer reads. Passed and failed reads were collapsed into per-sample datasets for this study based on the observation that $\sim 10 \%$ of reads could be recovered from failed read folders. Analysis of host-mapping RNA was deferred for this paper.

\section{3' bias}

All experiments demonstrated strong 3' coverage bias (Figures 2, 3). This was also seen in control ENO2 mRNA across all experiments (Supplemental Figure 1). The signal from the control template across experiments exhibited consistent decay in recovered read length. Evaluating the reduction in sequence across samples at consistent points (base 1,200 and 200) yielded an average loss of 65.55\% (SD = 11.08) of recovered 
transcripts per 1,000 bases. (Supplemental Table 2). Furthermore, when we removed the outlier NL4-3 sequencing run from the analysis, which was completed with previously used but still functional R9.4 RevC flow cells and RNA002, the average loss was $68.41 \%$ $(S D=0.6521)$

Splicing vs. ONT artifact vs. Structural variant

We observed reads with large deletions in a higher coverage experiment (NLAD8 in Figures $\mathbf{2 E}, \mathbf{3 H}$, and $\mathbf{3 I}$ ). Without DNA sequencing, it is difficult to tell whether these are at the genomic or transcriptomic level. That 1) these were a minority, and that 2) the exact start and stop points differed between these few reads are suggestive of the latter. A smaller deletion was observed in a region of NLAD8 outside of env (Supplemental Figure 5). This small deletion had a different mapping profile compared to homopolymer runs (Supplemental Figure 2). The large deletions occurred at similar locations across multiple reads. Splicing was also evident as clipped reads in most samples (Figure 2). Other experiments either did not have such transcriptional features or lacked coverage sufficient to observe these features.

\section{SNV calls from ONT Native RNA}

Samples with available reference genomes (with GenBank accessions) were manually evaluated for single nucleotide variants, including small insertions and deletions, under the conditions: 1 ) that there were at least 10 reads at a given locus; and 2) that the reference allele occurred less than or equal to $50 \%$ of the time. In the event that a putative SNV occurred over a deletion, the SNV was included in the set.

Supplemental Figures 4-7 include SNVs, small insertions, deletions. See Supplemental Table 3 for a list of likely SNVs directly called from native (ONT) RNA-seq. 
The region satisfying the above for 89.6 was $6,831-9,624$ (length $=2,793$ ) with respect to the reference 89.6 U39362.2. There were at least 45 SNVs in the version of 89.6 sequenced. The mutation rate, calculated as the number of SNVs divided by window length, was $45 / 2,793=0.01611171$. The region satisfying the above for 92 UG029 was $8,154-8,820$ (length $=666$ ) with respect to its reference AY713407.1. There were at least 7 SNVs in the version of 92 UG029 sequenced. The mutation rate was $7 / 666=$ 0.01051051. The region satisfying the above for BaL was 4,733-8,775 (length $=4,042$ ) with respect to its reference AY713409.1. There were at least 23 SNVs in the version of BaL sequenced. The mutation rate was $23 / 4,042=0.00569025$. The region satisfying the above for ELI was 7,105-9,176 (length $=2,071$ ) with respect to its reference K03454.1. There were at least 18 SNVs in the version of ELI sequenced. The mutation rate was $18 / 2,071=0.00869145$. The region satisfying the above for HIV1-SX was $5,385-9,624$ (length $=4,239$ ) with respect to a related reference pNL4-3 AF324493.2. However, HIV1$\mathrm{SX}$ is chimeric, with JR-FL's env region. Excluding env, there were no discernable differences between HIV1-SX and pNL4-3. The region satisfying the above for MN was $7,752-9,654$ (length $=1,902$ ) with respect to its reference M17449.1. There were at least 11 SNVs in the version of MN sequenced. The mutation rate was $11 / 1,902=0.00578339$. The region satisfying the above for NL4-3 was $8,169-9,625$ (length $=1,456$ ) with respect to its reference AF324493.2. There were at least 2 SNVs in the version of NL4-3 sequenced. The mutation rate was $2 / 1,456=0.00137363$. The region satisfying the above for NLAD8 was $1,474-9,626$ (length $=8,152$ ) with respect to its reference AF324493.2. There were at least 6 SNVs in the version of NLAD8 sequenced. NLAD8 was completely conserved (no obvious SNVs) in its env region compared to AD8 (Figure $3 \mathrm{H}$, Figure $3 \mathrm{I}$ ). The region satisfying the above for RHPA was $8,324-9,621$ (length $=1,297$ ) with respect to its 
reference JN944944.1. There was one SNV in the version of RHPA sequenced, g.8,570C>T, nonsynonymous $\mathrm{T}>\mathrm{M}$ in rev and nonsynonymous $\mathrm{R}>\mathrm{C}$ in env, with possible structural consequence. The mutation rate was $6 / 8,152=0.00073602$. Combined, the average mutation rate (excluding HIV-SX and RHPA) was 0.00698528, (SD =0.00535872). We noticed variants occurring in close proximity to other neighboring variants. These represent interesting cases of context-dependent variance, which we classify with the following system: Independent, Neighbor, Neighborhood, or Neighbor+Neighborhood to emphasize the points that adjacent bases can contribute to multiple amino acid changes, and that these are not immediately apparent when focusing on individual coding frames (See Supplemental Figure 8).

\section{Comparative transcriptomics}

HIV-1-mapped (AF033819.3 with minimap2) reads were assembled into contigs with Canu, a leading de novo long read genome assembler [10]. Contigs were generated for 89.6, 97USNG30, AD17, BaL, ELI, HIV1-SX, MN, NLAD8 (Supplemental Figures 9, 10). Contigs were fed into MAFFT server [15], and basic phylogenetic trees generated with default settings (Figure 4). For sequences with GenBank accessions with complete genomes, the tree was homogenous. With incomplete contigs, general relationships (such as similarity to NL4-3) were maintained. By mapping reads to references we observed that phasing (the ability to tie SNVs to individual viral chromosomal haplotypes) was possible with native RNA reads (Supplemental Figure 7). We wanted to test whether this could be leveraged to study viral transcript heterogeneity directly. All FASTQ were converted to FASTA, concatenated, made into a multisequence alignment, and visualized as a tree (Figure 4). Surprisingly, sample relationships were maintained despite per-read noise. 


\section{DISCUSSION}

\section{First complete full-length sequences from infectious HIV-1 strains.}

In this study we were able to completely [16] sequence the viral RNA genome of three HIV-1 strains (Figure 1). Here, we defined complete or full-coverage of full-length HIV-1 viral RNA as the region capable of sustaining viral replication with supplemental reverse transcription and integrase activity. This corresponds to the region from the $5^{\prime}$ LTR-mediated transcriptional start site (TSS) and the repeat region after the end of the U3 region of the $3^{\prime}$ LTR. Note that these dimensions are different than the dimensions of HIV1 provirus. To our knowledge, this is the first time the HIV-1 virus has been sequenced from the TSS to end of 3' LTR. As reported for HIV-1 in a DNA context [3], ONT surpassed the read length limitations of short read RNA-seq. Data were sufficient for SNV detection over regions with coverage $\geq 10$ reads, as well as direct de novo assembly from native RNA reads, with a maximal assembly length of 94\% from strain NLAD8.

\section{Mapping}

Historically lower per-read accuracy and high sequence variability across HIV isolates between and within patients represent two challenges to detecting native HIV reads. Interestingly, newer long read mappers such as minimap2 were robust to references used (Mapping quality $>50$ ). We saw slightly more recovered reads when we mapped our reads to references with greater homology (Figures $\mathbf{2 A}$ and $3 \mathrm{~A}$; Figures $\mathbf{2 C}$ and 3B; Figures 2G and 3C; Figures 2H and 3D; Figures 2K and 3F; Figures 2L and 3G;

Figures $\mathbf{2 M}$, 3H and 31; Figures $\mathbf{2 N}$ and $3 \mathrm{~J}$ ). We recommend using strain-specific references when possible, ideally sequence-verifying constructs used to make viruses in each lab to catch any lurking SNVs [3]. 
Coverage

These experiments represent a proof-of-concept demonstrating ONT's ability to cover HIV mRNA completely (Figures $2 \mathrm{~A}, \mathbf{2 E}, \mathbf{2 M}, \mathbf{3 A}, \mathbf{3 H}$, and $\mathbf{3 I}$ ), dependent on the titer of viruses used for library prep (Table 1). Neither cDNA nor cDNA PCR ONT libraries were evaluated in the present study. Coverage as read depth (total number of bases sequenced divided by reference length) was not informative due to strong 3' bias.

$3^{\prime}$ bias

We were able to recover full-length HIV RNA reads from 3 samples. This was in part due to strong $3^{\prime}$ bias inherent to the RNA002 library. The first adapter used by the RNA002 kit, RTA, selects for polyA [7]. There was relatively consistent coverage exhibiting a decrescendo pattern (with coverage decreasing distal from RTA ligation point), except near areas of known complex $2 \circ$ RNA structure [17]. The first strand cDNA step is supposed to ameliorate 20 RNA, but these might not be completely abrogated-for example, the region around the rev response element in Figure 2, 3 in higher-coverage experiments exhibited slight changes in average loss of recovered transcripts per 1,000 bases. A useful consequence of the $3^{\prime}$ bias is easier phasing between $5^{\prime}$ and $3^{\prime}$ LTR regions, which is difficult or impossible to tell apart using short read DNA [3] or classic RNA sequencing (Supplemental Figure 12). In fact, we did not encounter reads spanning full-length 5' LTR in any of our native RNA experiments, which were however present in HIV PCR + DNA ONT experiments [3].

Included in the RNA002 library kit is control RNA. By determining the percent loss per 1,000 bases, we recommend that at least 2,500x coverage over the $3^{\prime}$ LTR to guarantee observation of full-length HIV reads $(\sim 9 \mathrm{~kb})$ at a depth sufficient to call SNVs at the $5^{\prime}$ end of transcripts (with coverage $\sim 60 x$, which we recommend for Canu). At current 
sequencing throughput, this is equivalent to about one MinION RevD flow cell, with possibly multiple technical replicates of individual libraries. Note that higher-titer virus stocks were associated with higher coverage, although the sequencing time was not held constant.

\section{Splicing vs. ONT artifact vs. Structural variant}

In NLAD8 (Figures $\mathbf{2 M}, \mathbf{3 H}$, and $\mathbf{3 H I}$ ), there was high enough coverage toward the middle of HIV to see deletions relative to reference. These read deletions might be splicing, artifacts from library prep (with T4 DNA ligase), or due to previously observed HIV-1 deletion-prone integrants [18-20]. Note that these deletions are individual observations and not necessarily representative of the strains sequenced. A possible explanation supporting splicing includes observations of splicing events in a short read (cDNA) RNA-seq experiment (collected by Siarhei Kharytonchyk and submitted by Matthew Eckwahl). This incompletely published experiment was most likely using virus derived from pNL4-3, a commonly used lab-adapted clone of HIV-1 (Gener, unpublished). (See Supplemental Figure 12.) Mapping peaks also occurred at sites of known exons. On closer inspection, these reads exhibited clipping indicating their being part of a spliced read.

\section{SNV calls from ONT Native RNA}

Strain-specific reference mapping was performed on 89.6, 92UG029, BaL, ELI, MN, NL4-3, and NLAD8. Abundance of coverage allowed us to evaluate single nucleotide variants (SNVs), as well as small insertions and deletions, in areas with $\geq 10 x$ coverage (minimum recommended by Canu documentation). The average mutation rate across sequenced samples (Supplemental Table 3) was 0.00698528 . This meant that for each full-length HIV-1 virion (say with respect to the HIV-1 reference AF033819.3) one would 
expect $0.00698528 \times 9,181$ bases $=\sim 64$ SNVs, possibly contributing to altered protein products. We introduce neighbor and neighborhood nomenclature for SNVs to emphasize that variants occurring in information-dense regions can have complex contextdependent effects. What was demonstrated for HIV in its DNA form [3] also applies for HIV in its RNA form: SNVs happen. Sequencing the viral strains and cloned constructs used for experiments in a given laboratory remains important, because what is reported in a reference database for a given virus may not necessarily be exactly the same as the viruses propagated in a laboratory.

\section{Capturing variability in reference HIV-1 viruses}

HIV is the most studied human pathogen [3], and yet there were at least 144 SNVs across 9 HIV-1 reference strains. Some occurred in previously camouflaged [21] repetitive LTR regions, but most occurred in the gene bodies of sequenced strains, some contributing to alterations in protein-coding potential. In this work we leveraged sequencing multiple related strains with mapping to their predecessors to simulate true positive reads. Long read native RNA sequencing on the ONT MinION with R9.4.1 RevD flow cells was robust and sufficient to detect real sequence variability at the singlemolecule level, except for at areas in and around homopolymers. While not a complete solution yet, long read native RNA sequencing offers the ability to tie SNVs together, to define segments of HIV haplotypes directly, and to move away from the concept of quasispecies $[1,22]$ toward defining real viral haplotypes. An extension of this capability is the ability to perform comparative transcriptomics within hours after sample acquisition (Figure 4), including determining drug resistance profiles based on existing public databases (Supplemental Figure 11) [23,24]. 
Homopolymers

As previously reported $[3,25]$ for DNA ONT, per-read variability in ONT data was higher near homopolymers (runs of the same base) (Supplemental Figure 2). ONT is also known for lower per-base accuracy compared to short read next generation platforms. These may be overcome by evaluating neighbor SNVs (Supplemental Figure 3). New developments include improved basecalling models and a new double-header R10 nanopore, which has been showing improved handling of homopolymers, with slight drop in per-read accuracy. When comparing sequences, the errors in homopolymers are observed consistently across reads, and become negligible. We were able to compare individual reads from mostly incomplete virion genomes with simple cladistics and reads successfully grouped based on sample identity (Figure 4).

\section{Suitability of ONT for HIV sequencing}

Comparing Classic RNA-seq to Native RNA-seq, each technology has its pros and cons. Classic RNA-seq, usually cDNA synthesis optionally followed by PCR, can be sensitive, specific, and cheaper with newer higher throughput sequencers (example: Illumina, BGI). While PCR enhances sensitivity (to this day, it is important for detecting viral sequences at low copy numbers $[26,27])$, a downside is that captured information can be lost if PCR is used during library prep (Supplemental Figure 12). Direct cDNA, or using long read DNA sequencing (PacBio [2], ONT), can also recuperate sequence information from samples with relatively higher throughput than native RNA-seq in its current implementation. It will be important to address sequencing throughput and sequence biases when developing this nascent technology. At present, it is possible to sequence and compare viral isolates as contigs and for the first time as individual virus genomes as individual reads (Figure 4), as well as to determine drug resistance profiles 
from contigs (Supplemental Figure 11) or individual reads. A benefit that is unique to native RNA sequencing on ONT devices is the ability to detect base modifications as well. Work is ongoing to evaluate the raw signal from these experiments, and to evaluate new ONT RNA analysis tools for use with HIV.

\section{Closing the distance on HIV-1 with longer reads}

Current limitations of the approaches used in the present work to study HIV RNA are similar to those reported for HIV DNA [3], and include: 1) the cost of long-read sequencing, compared to the cheaper short read DNA sequencing (as in classic RNA-seq); 2) long RNA extraction methods in diseased tissue (Gener, unpublished); and 3) lower per-base accuracy (mid 90's vs. 98-99\%), including difficulty near homopolymers (Supplemental Figure 2). Furthermore, a unique major limitation to RNA sequencing is 4): $3^{\prime}$ bias. As the price of long-read sequencing continues to decrease, and as the technology improves, the cost of obtaining usable data from native RNA long read sequencing will become negligible compared to the ability to answer new questions. Classic RNA-seq had a problem with 3 ' bias, but the issue was eventually overcome [28]. Future work will move toward the goal of capturing higher-coverage fuller glimpses of each HIV viral mRNA, including virion genomes in in vivo HIV models and from patient samples. Long read sequencing is an important emerging tool defining the post-scaffold transcriptomic era, allowing for the characterization of functional units at the intersection between host and pathogen transcriptomes. 


\section{ACKNOWLEDGEMENTS}

ARG conceived of this project and performed all experiments. ARG and JK analyzed results and wrote the manuscript. The authors would like to thank Dr. Sue Ellen Crawford for earlier involvement and Victoria Rose Tenge for advice, technical assistance, and critical reading of the manuscript.

This work was funded in part by institutional support from Baylor College of Medicine; the Human Genome Sequencing Center, BCM; private funding by East Coast Oils, Inc., Jacksonville, Florida, and ARG's own private funding, including Student Genomics (manuscripts in prep). Compute resources from the Computational and Integrative Biomedical Research Center at BCM ("sphere" cluster managed by Dr. Steven Ludtke) and the Department of Molecular and Human Genetics at BCM ("taco" cluster managed by Mr. Tanner Beck, Mr. Christopher Michael Holder, and Dr. Charles Lin) greatly facilitated the completion of this work. ARG has also received the PFLAG of Jacksonville scholarship for multiple years. The authors would like to thank Dr. David Raul Murdock in Dr. Brendan Lee's Laboratory and Mr. Alexander Robert Kneubehl in Dr. Job E. Lopez's Laboratory for making their MinION devices available. The authors would like to thank the Mary K. Estes Laboratory at BCM for making available their Kingfisher instrument for viral RNA isolation. This work was also supported in part by NIH grant AI116167 to JTK.

Infectious virus stocks of HIV-1 (SF162, 97USNG30, MN, ADA, 92UG038, 92UG029, ELI, JR-FL, and BaL) or molecular clones (NL4-3, NLAD8, RHPA, AD17) were obtained from M. Martin, R. Collman, E.O. Freed, B. Hahn, G. Shaw, J. Levy, D. Ellenberger, ,P. Sullivan, R.B. Lal, R.C. Gallo, H. Gendleman, I.S.Y. Chen, S. Gartner, M. Popovic, and the UN AIDS 
Reagent and Reference Program. HIV1-SX was provided by M.R. Ferguson and W.A.

O’Brien.

ARG would like to thank members of the Paul E. Klotman Laboratory at Baylor

College of Medicine, including Dr. Paul E. Klotman, Dr. Deborah P. Hyink (who

thoughtfully helped to edit the manuscript), Taneasha Washington, and former members

Dr. Gokul C. Das and Alexander Batista.

This work is dedicated to the memory of Dr. James "Jim" E. Maruniak, an

outstanding virologist, educator, family man, role-model, and friend. 
REFERENCES

1 Lauring AS, Andino R. Quasispecies Theory and the Behavior of RNA Viruses. PLoS Pathog 2010; 6. doi:10.1371/journal.ppat.1001005

2 Ocwieja KE, Sherrill-Mix S, Mukherjee R, Custers-Allen R, David P, Brown M, et al. Dynamic regulation of HIV-1 mRNA populations analyzed by single-molecule enrichment and long-read sequencing. Nucleic Acids Res 2012; 40:10345-10355.

3 Gener AR. Full-coverage sequencing of HIV-1 provirus from a reference plasmid. bioRxiv 2019; :611848.

4 Graf EH, Mexas AM, Yu JJ, Shaheen F, Liszewski MK, Di Mascio M, et al. Elite suppressors harbor low levels of integrated HIV DNA and high levels of 2-LTR circular HIV DNA compared to HIV+ patients on and off HAART. PLoS Pathog 2011;

7. doi:10.1371/journal.ppat.1001300

5 Cuesta I, Mari A, Ocampo A, Miralles C, Pérez-castro S, Thomson MM. Sequence Analysis of In Vivo -Expressed HIV-1 Spliced RNAs Reveals the Usage of New and Unusual Splice Sites by Viruses of Different Subtypes. 2016; :1-24.

6 Klotman M, Kim S, Buchbinder A, DeRossi A, Baltimore D, Wong-Staal F. Kinetics of expression of multiply spliced RNA in early human immunodeficiency virus type 1 infection of lymphocytes and monocytes. Proc Natl Acad Sci U S A 2006; 88:50115015.

7 Workman RE, Tang AD, Tang PS, Jain M, Tyson JR, Zuzarte PC, et al. Nanopore native RNA sequencing of a human poly(A) transcriptome. bioRxiv 2018; :459529.

8 Keller MW, Rambo-Martin BL, Wilson MM, Ridenour CA, Shepard SS, Stark TJ, et al. Direct RNA Sequencing of the Coding Complete Influenza A Virus Genome. Sci Rep 2018; 8:14408.

9 Misra A, Gleeson E, Wang W, Ye C, Zhou P, Kimataa JT. GlycosylPhosphatidylinositol-Anchored Anti HIV Env Single-Chain Variable Fragments Interfere with HIV-1 Env Processing and Viral Infectivity. J Virol 2018; 92:1-15.

10 Walenz BP, Koren S, Bergman NH, Phillippy AM, Miller JR, Berlin K. Canu: scalable and accurate long-read assembly via adaptive $\mathrm{k}$-mer weighting and repeat separation. Genome Res 2017; 27:722-736.

11 Wysoker A, Fennell T, Marth G, Abecasis G, Ruan J, Li H, et al. The Sequence Alignment/Map format and SAMtools. Bioinformatics 2009; 25:2078-2079.

12 García-Alcalde F, Okonechnikov K, Carbonell J, Cruz LM, Götz S, Tarazona S, et al. Qualimap: evaluating next-generation sequencing alignment data. Bioinformatics 2012; 28:2678-2679.

13 Li H. Minimap2: pairwise alignment for nucleotide sequences. Bioinformatics 2018; 34:3094-3100.

14 Afgan E, Baker D, van den Beek M, Blankenberg D, Bouvier D, Čech $M$, et al. The Galaxy platform for accessible, reproducible and collaborative biomedical analyses: 2016 update. Nucleic Acids Res 2016; 44:W3-W10.

15 Katoh K, Rozewicki J, Yamada KD. MAFFT online service: multiple sequence alignment, interactive sequence choice and visualization. Brief Bioinform 2017; 20:1160-1166.

16 Ladner JT, Beitzel B, Chain PSG, Davenport MG, Donaldson E, Frieman M, et al. Standards for Sequencing Viral Genomes in the Era of High-Throughput Sequencing. MBio 2014; 5:e01360-14. 
17 HIV-1 RNA Structures Index. HIV Seq. Database. https://www.hiv.lanl.gov/content/sequence/STRUCTURE/RNA.HTML

18 Ho Y-C, Shan L, Hosmane NN, Wang J, Laskey SB, Rosenbloom DIS, et al. Replication-Competent Noninduced Proviruses in the Latent Reservoir Increase Barrier to HIV-1 Cure. Cell 2013; 155:540-551.

19 Bruner KM, Murray AJ, Pollack RA, Soliman MG, Sarah B, Capoferri AA, et al. Defective proviruses rapidly accumulate during acute HIV-1 infection. Nat Med 2017; 22:1043-1049.

20 Pollack RA, Jones RB, Pertea M, Benko E, Siliciano RF, Ho Y. Defective HIV-1 Proviruses Are Expressed and Can Be Recognized by Cytotoxic T Lymphocytes, which Shape the Proviral Landscape Article Defective HIV-1 Proviruses Are Expressed and Can Be Recognized by Cytotoxic T Lymphocytes, which Shape the Proviral Landscape. Cell Host Microbe 2017; 21:494-506.e4.

21 Ebbert MTW, Jensen TD, Jansen-West K, Sens JP, Reddy JS, Ridge PG, et al. Systematic analysis of dark and camouflaged genes reveals disease-relevant genes hiding in plain sight. Genome Biol 2019; 20:97.

22 Pandit A, Boer RJ De. Reliable reconstruction of HIV-1 whole genome haplotypes reveals clonal interference and genetic hitchhiking among immune escape variants. 2014; :1-15.

23 Rhee S, Gonzales MJ, Kantor R, Betts BJ, Ravela J, Shafer RW. Human immunodeficiency virus reverse transcriptase and protease sequence database. 2003; 31:298-303.

24 Shafer RW. Rationale and Uses of a Public HIV Drug-Resistance Database. 2006; 94305:51-58.

25 Loman NJ, Quick J, Simpson JT. A complete bacterial genome assembled de novo using only nanopore sequencing data. Nat Methods 2015; 12:733-735.

26 Worobey M, Watts TD, McKay RA, Suchard MA, Granade T, Teuwen DE, et al. 1970s and 'Patient 0' HIV-1 genomes illuminate early HIV/AIDS history in North America. Nature 2016; 539:98.

27 Yamaguchi J, McArthur C, Vallari A, Sthreshley L, Cloherty GA, Berg MG, et al. Complete genome sequence of CG-0018a-01 establishes HIV-1 subtype L. JAIDS J Acquir Immune Defic Syndr 9000; Publish Ahead of

Print.https://journals.Iww.com/jaids/Fulltext/publishahead/Complete_genome_se quence_of_CG_0018a_01.96307.aspx

28 Sheng K, Cao W, Niu Y, Deng Q, Zong C. Effective detection of variation in single-cell transcriptomes using MATQ-seq. Nat Methods 2017; 14:267.

29 Collman R, Balliet JW, Gregory SA, Friedman H, Kolson DL, Nathanson N, et al. An Infectious Molecular Clone of an Unusual Macrophage- Tropic and Highly Cytopathic Strain of Human Immunodeficiency Virus Type 1 A /. 1992; 66:75177521.

30 Sullivan PS, Do AN, Ellenberger D, Pau C, Paul S, Robbins K, et al. Human Immunodeficiency Virus ( HIV ) Subtype Surveillance of African-Born Persons at Risk for Group O and Group N HIV Infections in the United States. 1997; :463-469.

31 Parrish NF, Gao F, Li H, Giorgi EE, Barbian HJ, Parrish EH, et al. Phenotypic properties of transmitted founder HIV-1. 2013; 110:6626-6633.

32 Gendelman HE, Baca LM, Kubrak CA, Genis P, Burrous S, Friedman RM, et al. Induction of IFN-alpha in peripheral blood mononuclear cells by HIV-infected 
monocytes. Restricted antiviral activity of the HIV-induced IFN. J Immunol 1992;

148:422-429.

33 Gendelman HE, Orenstein JM, Martin MA, Ferrua C, Mitra R, Phipps T, et al. Efficient isolation and propagation of human immunodeficiency virus on recombinant colony-stimulating factor 1-treated monocytes. J Exp Med 1988; 167:1428-1441.

34 Westervelt P, Gendelmant HE, Ratner LEE. Identification of a determinant within the human immunodeficiency virus 1 surface envelope glycoprotein critical for productive infection of primary monocytes. 1991; 88:3097-3101.

35 Gartner S, Markovits P, Markovitz DM, Kaplan MH, Gallo RC, Popovic M. The role of mononuclear phagocytes in HTLV-III/LAV infection. Science 1986; 233:215-219.

36 Alizon M, Wain-Hobson S, Montagnier L, Sonigo P. Genetic variability of the AIDS virus: nucleotide sequence analysis of two isolates from African patients. Cell 1986; 46:63-74.

37 Brien WAO, Koyanagit Y, Namazie A, Zhao J, Diagne A, Idler K, et al. HIV -1 tropism for mononuclear phagocytes can be determined by regions of gp 120 outside the CD4-binding domain. 1990; 348:69-73.

38 Koyanagi Y, Miles S, Mitsuyasu RT, Merrill JE, Vinters H V, Chen IS. Dual infection of the central nervous system by AIDS viruses with distinct cellular tropisms. Science 1987; 236:819-822.

39 Koyanagi Y, O'Brien WA, Zhao JQ, Golde DW, Gasson JC, Chen IS. Cytokines alter production of HIV-1 from primary mononuclear phagocytes. Science 1988; 241:1673-1675.

40 Gallo RC, Salahuddin SZ, Popovic M, Shearer GM, Kaplan M, Haynes BF, et al. Frequent detection and isolation of cytopathic retroviruses (HTLV-III) from patients with AIDS and at risk for AIDS. Science 1984; 224:500-503.

41 Shaw GM, Hahn BH, Arya SK, Groopman JE, Gallo RC, Wong-Staal F. Molecular characterization of human T-cell leukemia (lymphotropic) virus type III in the acquired immune deficiency syndrome. Science 1984; 226:1165-1171.

42 Freed EO, Englund G, Martin MA. Role of the Basic Domain of Human Immunodeficiency Virus Type 1 Matrix in Macrophage Infection. 1995; 69:39493954.

43 Adachi A, Gendelman HE, Koenig S, Folks T, Willey R, Rabson A, et al. Production of acquired immunodeficiency syndrome-associated retrovirus in human and nonhuman cells transfected with an infectious molecular clone. J Virol 1986; 59:284-91.

44 Keele BF, Giorgi EE, Salazar-gonzalez JF, Decker JM, Pham KT, Salazar MG, et al. Identification and characterization of transmitted and early founder virus envelopes in primary HIV-1 infection. 2008; 105.

45 Ochsenbauer C, Edmonds TG, Ding H, Keele BF, Decker J, Salazar MG, et al. Generation of Transmitted / Founder HIV-1 Infectious Molecular Clones and Characterization of Their Replication Capacity in CD4 T Lymphocytes and Monocyte-Derived Macrophages. 2012; :2715-2728.

46 Salazar-gonzalez JF, Bailes E, Pham KT, Salazar MG, Guffey MB, Keele BF, et al. Deciphering Human Immunodeficiency Virus Type 1 Transmission and Early Envelope Diversification by Single-Genome Amplification and Sequencing $\square$. 2008; 82:3952-3970. 
47 Salazar-gonzalez JF, Salazar MG, Keele BF, Learn GH, Giorgi EE, Li H, et al. Genetic identity, biological phenotype, and evolutionary pathways of transmitted / founder viruses in acute and early HIV-1 infection. 2009; 206:1273-1289.

48 Cheng-Mayer C, Levy JA. Distinct biological and serological properties of human immunodeficiency viruses from the brain. Ann Neurol 1988; 23:S58-S61.

49 Robinson JT, Thorvaldsdóttir H, Winckler W, Guttman M, Lander ES, Getz1 G, et al. Integrative genomics viewer. Nat Biotechnol 2011; 29:24-26.

50 Kim D, Langmead B, Salzberg SL. HISAT : a fast spliced aligner with low memory requirements. 2015; 12. doi:10.1038/nmeth.3317 
bioRxiv preprint doi: https://doi.org/10.1101/845610; this version posted November 19, 2019. The copyright holder for this preprint (which was not certified by peer review) is the author/funder. All rights reserved. No reuse allowed without permission.

\section{TABLES \& FIGURES}


Table 1: Fifteen HIV-1 strains used in this study.

\begin{tabular}{|c|c|c|c|c|c|c|}
\hline HIV-1 Strain & $\mathrm{TCID}_{50} / \mathrm{ml}$ & Reference & Tropism & GenBank & ARP & Note \\
\hline 89.6 & $2.5 \times 10^{5}$ & [29] & R5 and $X 4$ & U39362.2 & 1966 & Passaged molecular clone. \\
\hline 92UG029 & $1.6 \times 10^{4}$ & None & $\mathrm{X} 4$ & AY713407.1 & 1650 & $\begin{array}{l}\text { Passaged isolate. Group M, } \\
\text { subtype A. }\end{array}$ \\
\hline 92UG038 & $4.2 \times 10^{5}$ & None & $\mathrm{X} 4$ & NA & 1744 & $\begin{array}{l}\text { Passaged isolate. Group M, } \\
\text { subtype D. }\end{array}$ \\
\hline 97USNG30 & $1.0 \times 10^{6}$ & [30] & ND & NA & 4115 & $\begin{array}{l}\text { Passaged isolate. Group M, } \\
\text { subtype C. }\end{array}$ \\
\hline AD17 & $1.0 \times 10^{4}$ & {$[31]$} & R5 & NA & 12423 & \begin{tabular}{|l} 
Transfected. \\
Transmitted/founder (T/F) HIV- \\
1 (Group M) subtype B \\
infectious molecular clone. \\
\end{tabular} \\
\hline ADA & $4.1 \times 10^{3}$ & {$[32-34]$} & R5 & NA & 416 & $\begin{array}{l}\text { Passaged isolate. Group M, } \\
\text { subtype B. }\end{array}$ \\
\hline BaL & $2.6 \times 10^{5}$ & [35] & R5 & AY713409 & 510 & $\begin{array}{l}\text { Passaged clone. Group M, } \\
\text { subtype B. }\end{array}$ \\
\hline ELI & $2.6 \times 10^{4}$ & {$[36]$} & $\mathrm{X} 4$ & K03454.1 & 2521 & $\begin{array}{l}\text { Passaged isolate. Group M, } \\
\text { subtype D. }\end{array}$ \\
\hline HIV1-SX & $1.0 \times 10^{5}$ & [37] & R5 & NA & NA & $\begin{array}{l}\text { Passaged clone. HIV-1SX is } \\
\text { NL4-3 with env of JR-FL. }\end{array}$ \\
\hline$J R-F L$ & $2.6 \times 10^{5}$ & {$[37-39]$} & R5 & NA & 395 & $\begin{array}{l}\text { Passaged isolate. Group, M, } \\
\text { subtype B. }\end{array}$ \\
\hline MN & $1.0 \times 10^{3}$ & {$[40,41]$} & $\mathrm{X} 4$ & M17449.1 & 317 & $\begin{array}{l}\text { Passaged isolate. Group M, } \\
\text { subtype B. }\end{array}$ \\
\hline NLAD8 & $1.0 \times 10^{6}$ & {$[42]$} & R5 & NA & 11346 & $\begin{array}{l}\text { Transfected. NL4-3 with env of } \\
\text { AD8 (AF004394.1). }\end{array}$ \\
\hline NL4-3 & $2.6 \times 10^{5}$ & [43] & $\mathrm{X} 4$ & AF324493.2 & 114 & $\begin{array}{l}\text { Transfected. Chimeric. Source } \\
\text { of Pro Virus: NY5 }\left(5^{\prime}\right) \text { and LAV } \\
\left(3^{\prime}\right) \text { cloned directly from } \\
\text { genomic DNA. }\end{array}$ \\
\hline RHPA & $1 \times 10^{3}$ & {$[44-47]$} & R5 & JN944944.1 & 11744 & $\begin{array}{l}\text { Transfected. } \\
\text { Transmitted/founder (T/F) HIV- } \\
1 \text { (Group M) subtype B } \\
\text { infectious molecular clone. }\end{array}$ \\
\hline SF162 & $2.6 \times 10^{4}$ & {$[48]$} & R5 & NA & 276 & Passaged isolate. Subtype B \\
\hline
\end{tabular}

Abbreviations: TCID = Tissue culture Infectious Dose. $\mathrm{R} 5=$ CCR5. X4 = CXCR4. NA = not applicable. ND = not done. Contents of "Note" and "Tropism" columns summarized from the NIH AIDS Reagent and Reference Program (ARP; https://aidsreagent.org). GenBank accessions reported only for strains with complete genomes available. 
Figure 1A: Read lengths for HIV-1-mapping reads sequenced in this study.

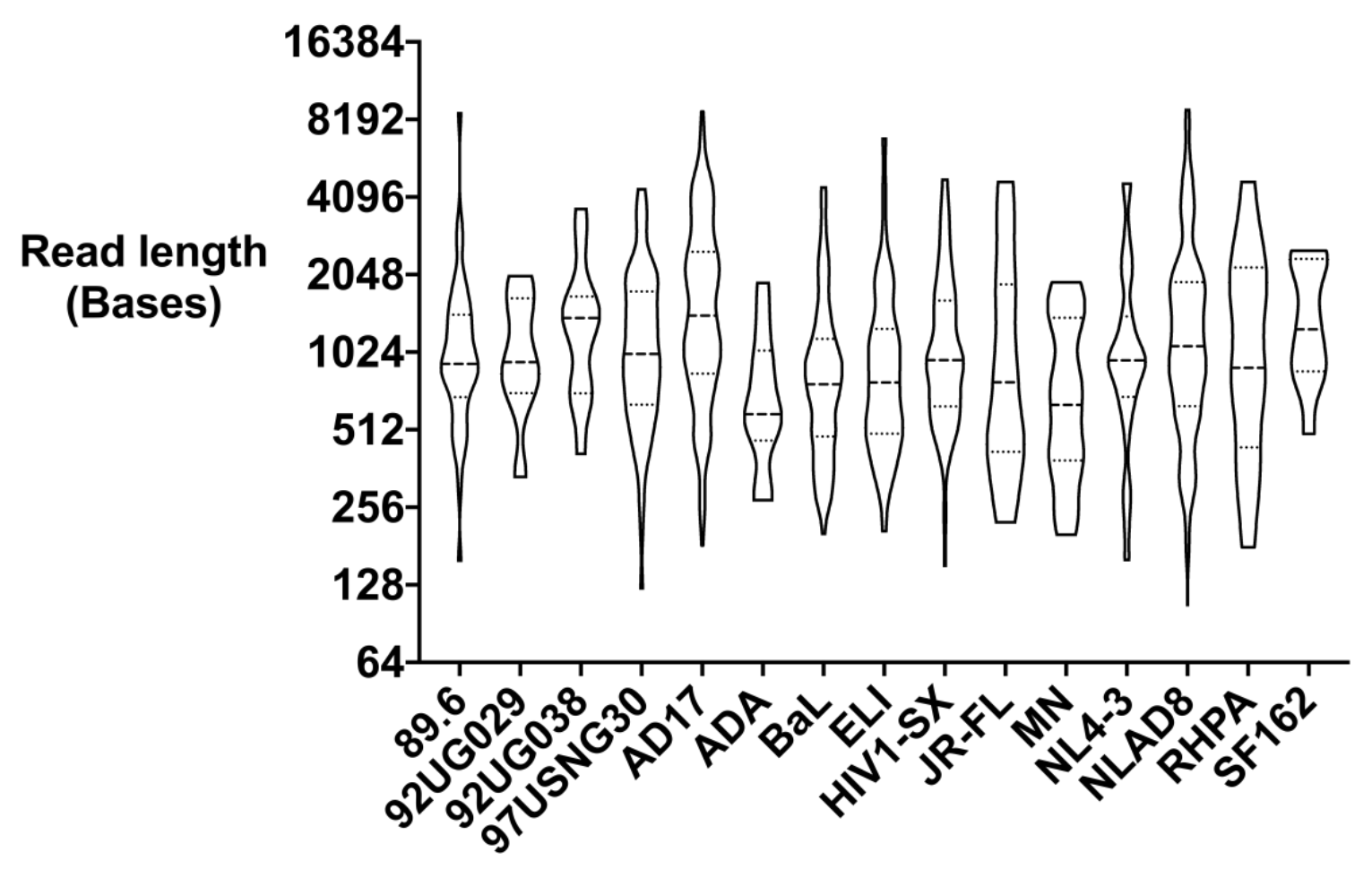

Violin plot of read length. $\log _{2}$-scaled. Median (big dash) and quartiles (little dash). All

native RNA reads are longer than most short DNA-seq reads. Note: Reads from multiple

runs either live basecalled or skipped reads basecalled afterward were collapsed. Per-run info is summarized in Supplemental Table 1. 
Figure 1B: Read mean quality scores for HIV-1-mapping reads sequenced in this study.

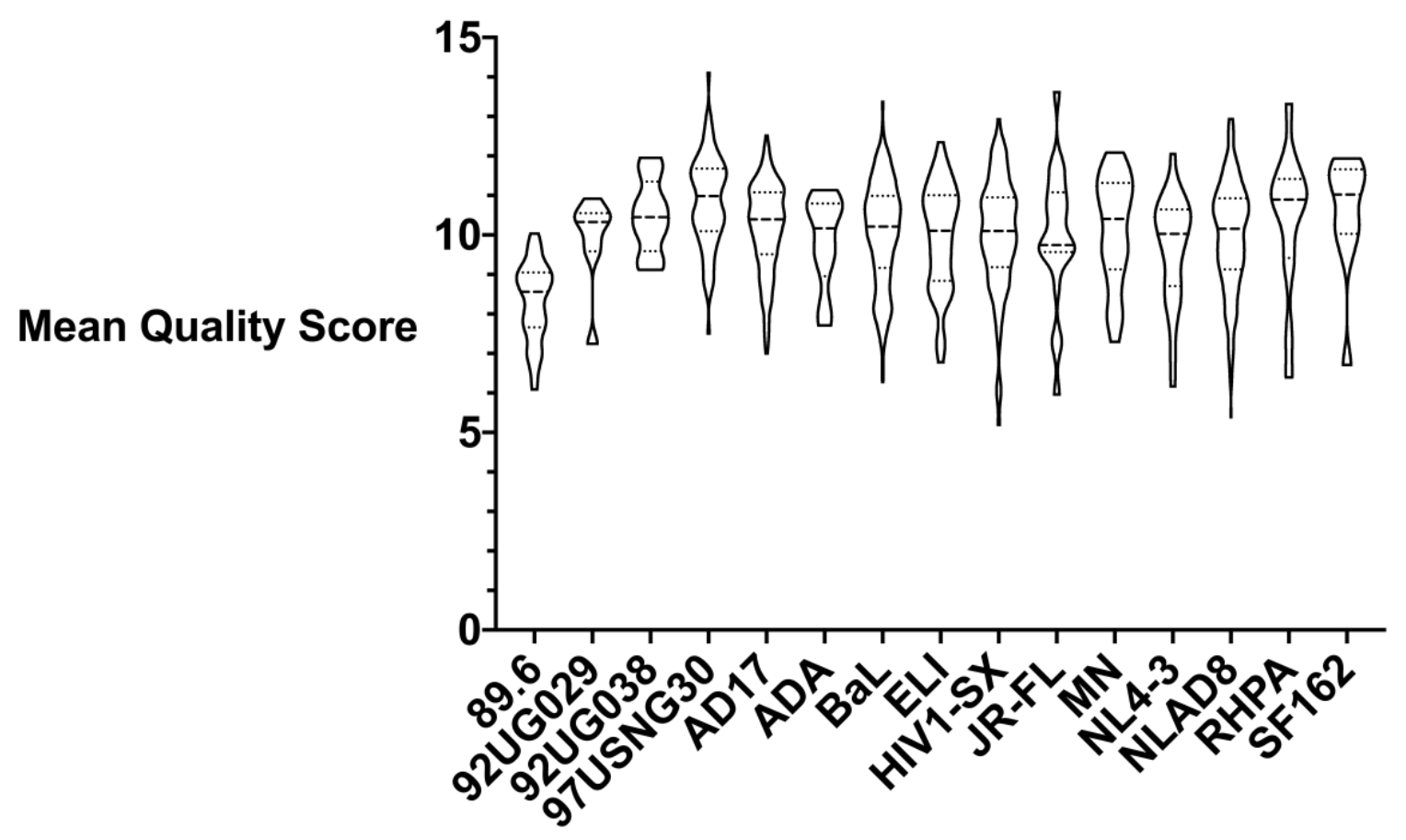

Violin plot of quality score. Median (big dash) and quartiles (little dash). Note: Reads from multiple runs either live basecalled or skipped reads basecalled afterward were collapsed. Per-run info is summarized in Supplemental Table 1. 
Figure 2A: 89.6

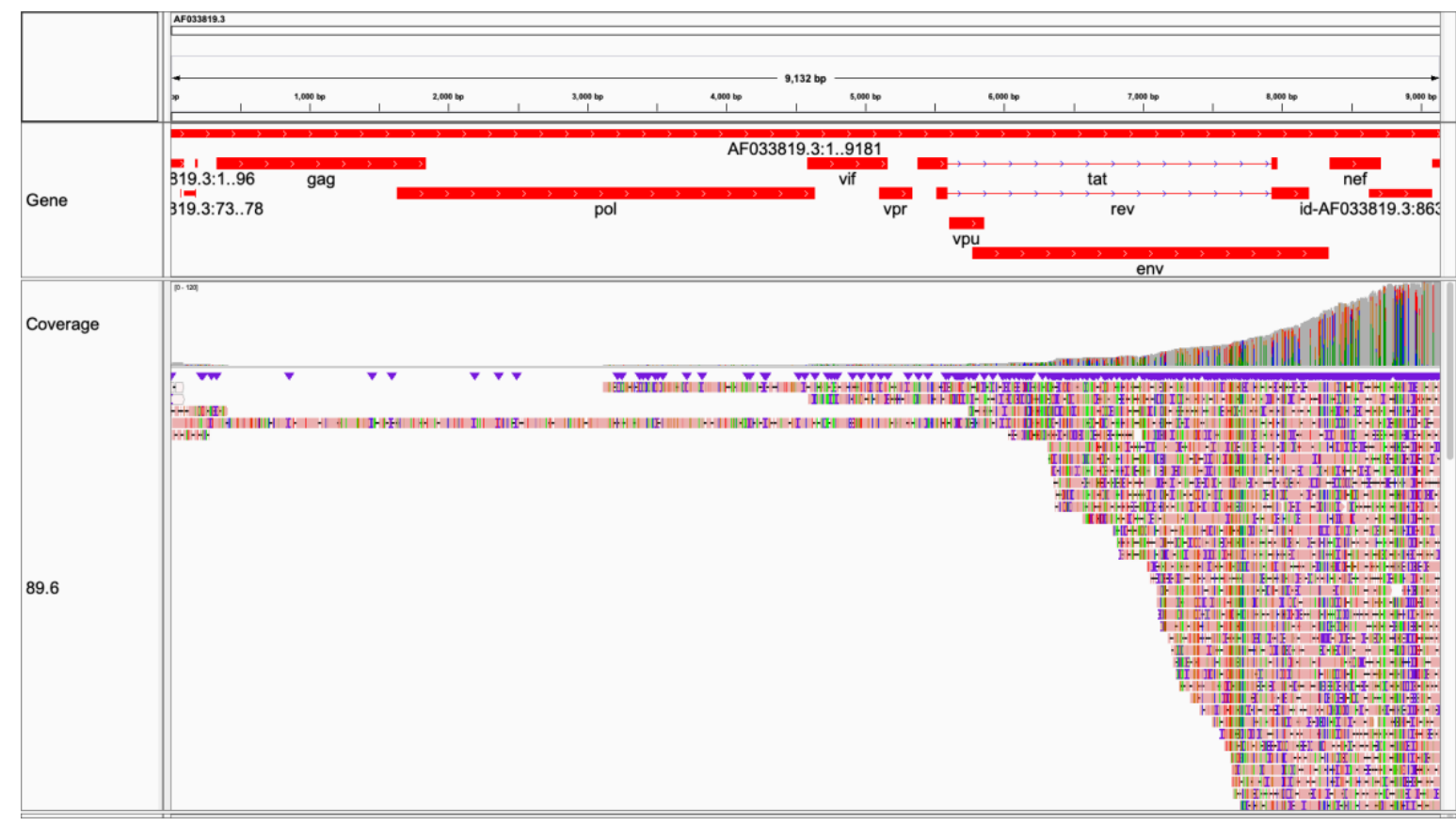

Full-coverage $(9,166 / 9,181 \sim 99.8 \%)$ achieved. Pink denotes forward strand orientation.

Colors (Red, Green, Gold, Blue) in reads denote differences from reference, not

necessarily read mismatches. Purple and black denote insertions and deletions in the

reads, which may or may not be errors in individual reads. Mapping to reference was

used to evaluate coverage across multiple HIV-1 strains. Note slight change in slope over

RRE. Visualized in Integrative Genomics Viewer [49]. Coverage over env possibly sufficient for cladistics (Figure 4). 
Figure 2B: 92 UG029

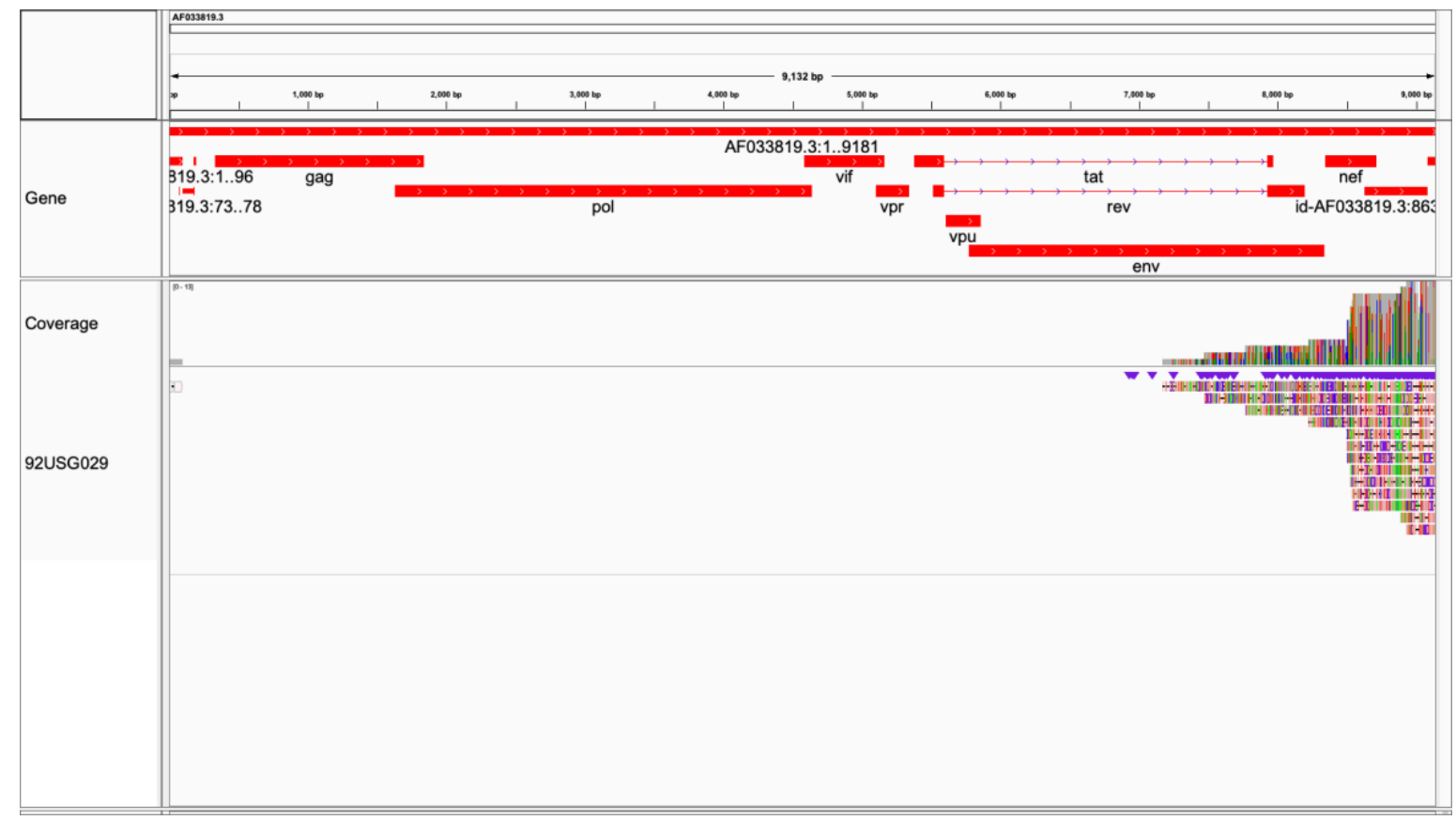

Lower coverage $(2,007 / 9,181 \sim 21.9 \%)$ achieved. Visualized in Integrative Genomics Viewer

[49]. Coverage over env insufficient for cladistics. 
Figure 2C: 92 UG038

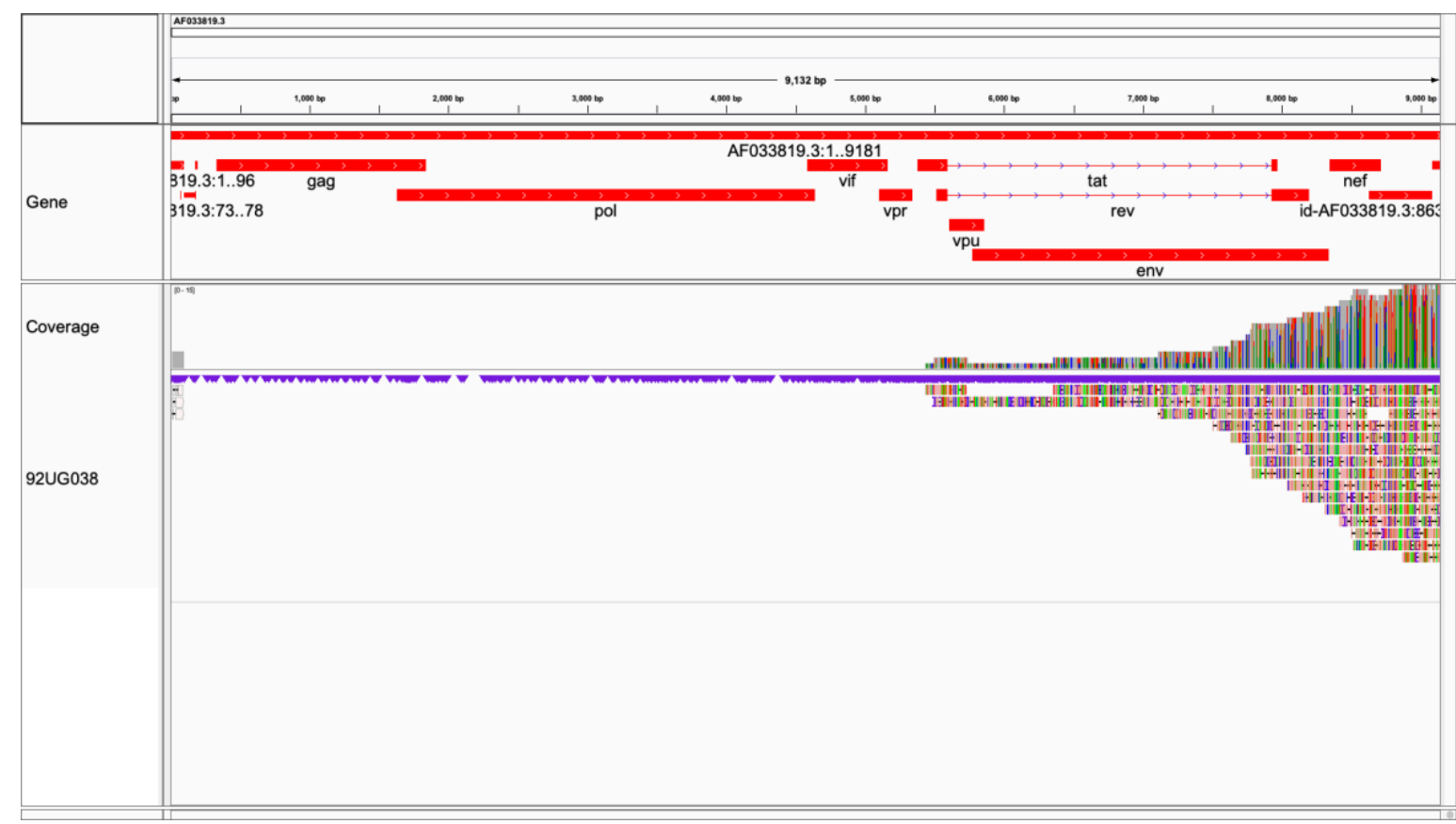

Moderate coverage $\left(3,678 / 9,181^{\sim} 40.1 \%\right)$ achieved. Note slight change in slope over RRE.

Visualized in Integrative Genomics Viewer [49]. Coverage over env insufficient for

cladistics. 
Figure 2D: 97USNG30

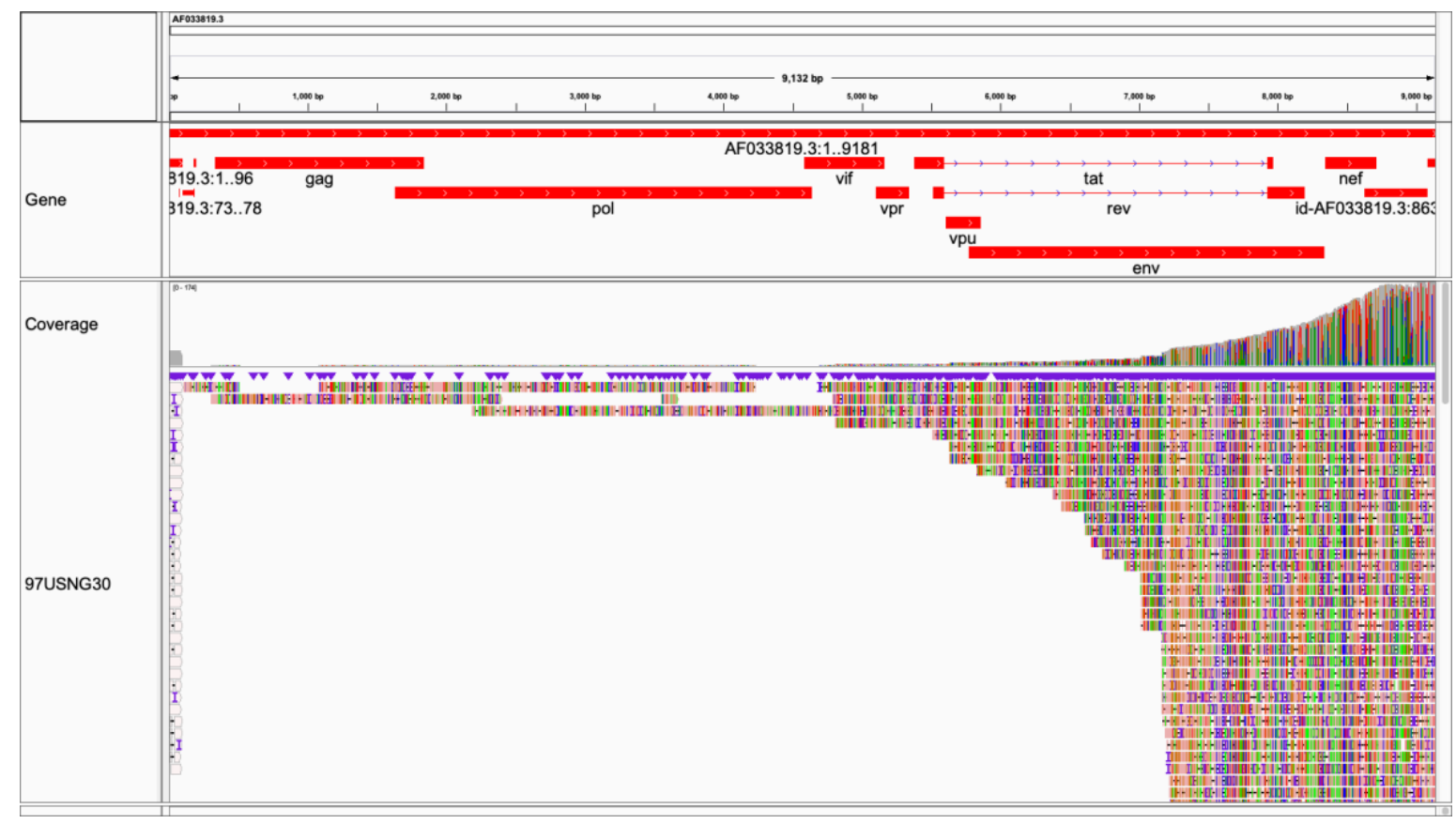

Majority coverage $(6,992 / 9,181 \sim 76.2 \%)$ achieved. Note slight change in slope over RRE.

Visualized in Integrative Genomics Viewer [49]. Coverage over env sufficient for cladistics

\section{(Figure 4).}


Figure 2E: AD17

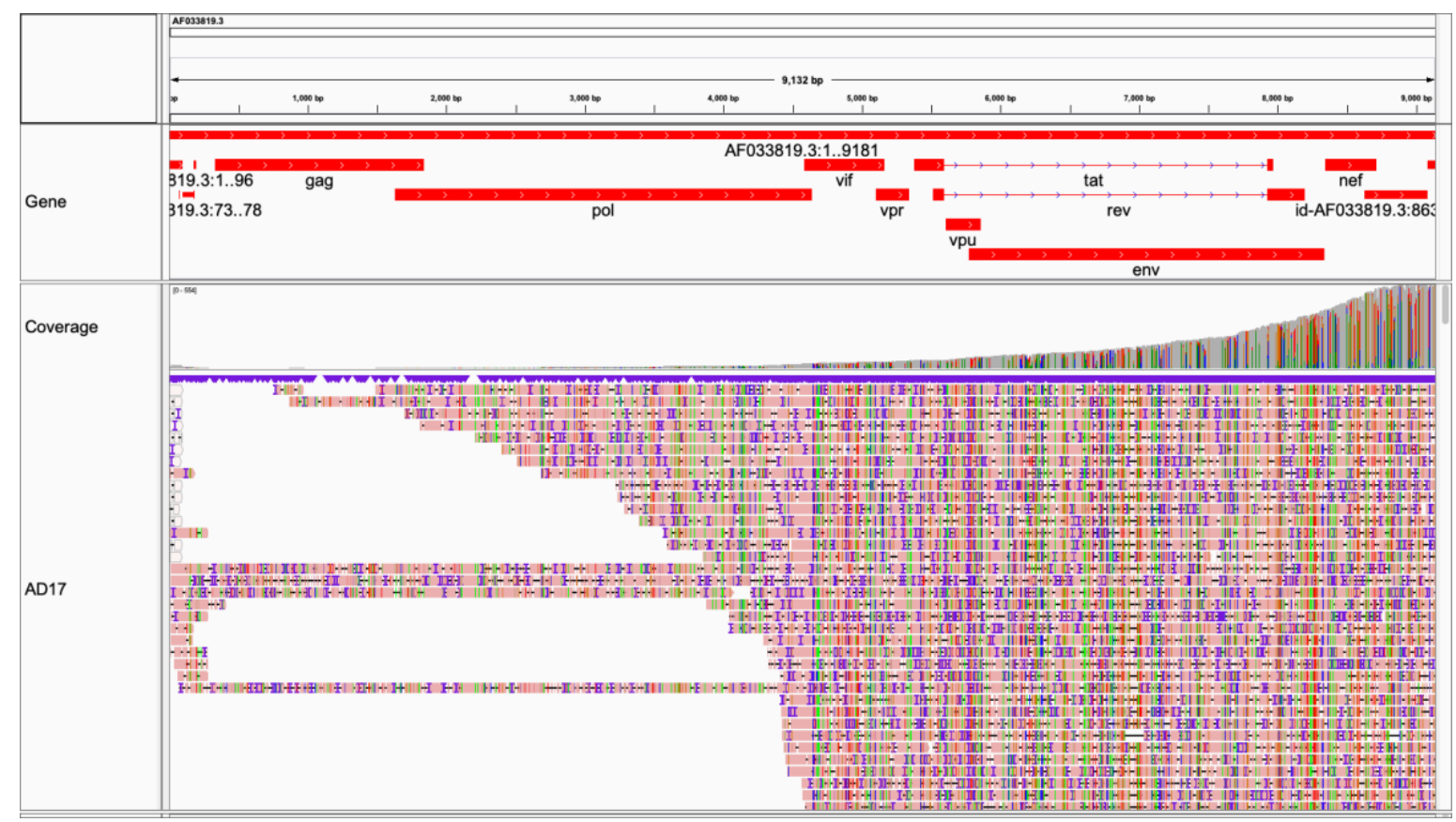

Full-length coverage $(9,164 / 9,181 \sim 99.8 \%)$ achieved. Visualized in Integrative Genomics

Viewer [49]. Coverage over env sufficient for cladistics (Figure 4). 
Figure 2F: ADA

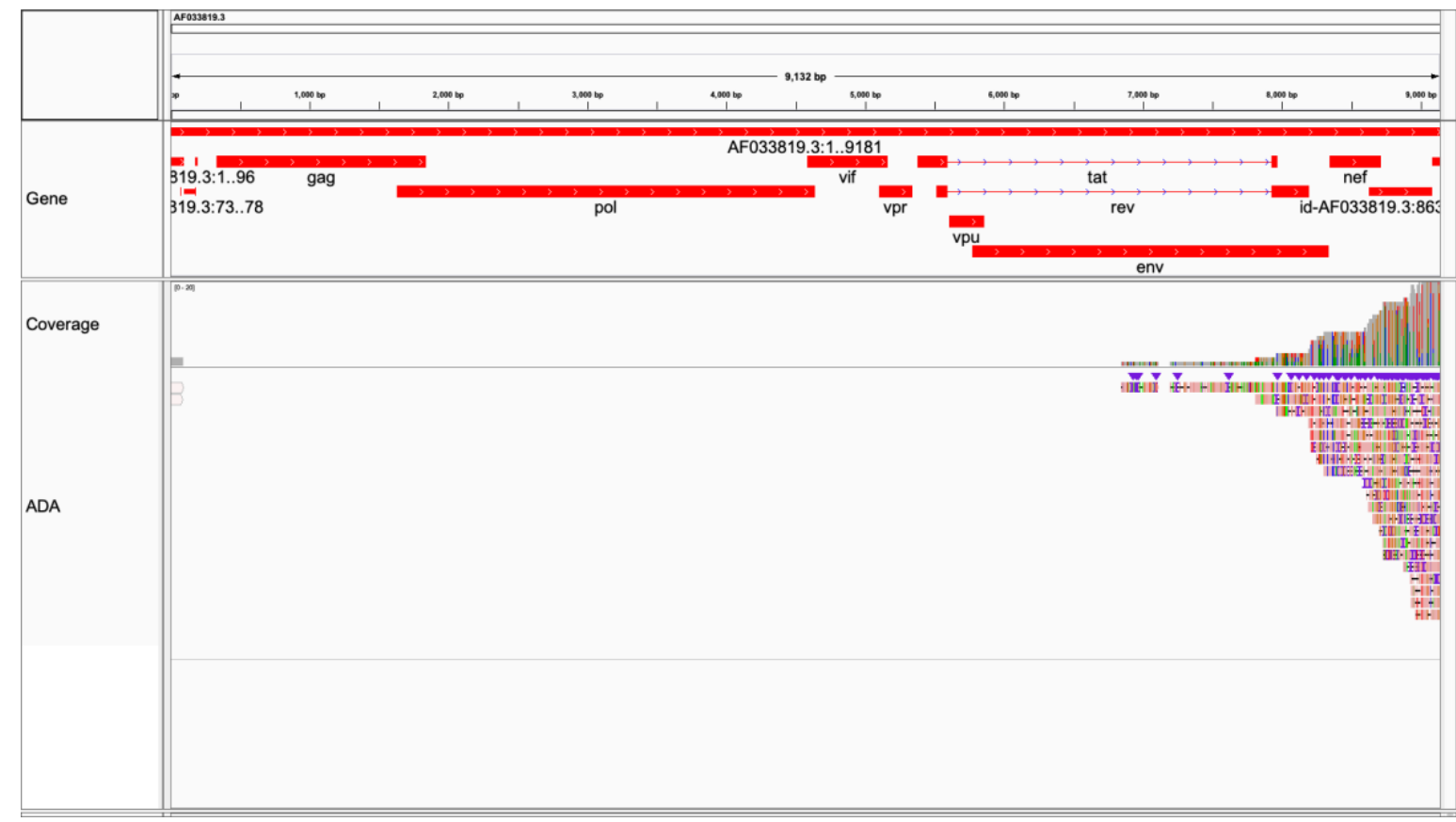

Lower coverage $(1,981 / 9,181 \sim 21.6 \%)$ achieved. Visualized in Integrative Genomics Viewer

[49]. Coverage over env insufficient for cladistics (Figure 4). 
Figure 2G: BaL

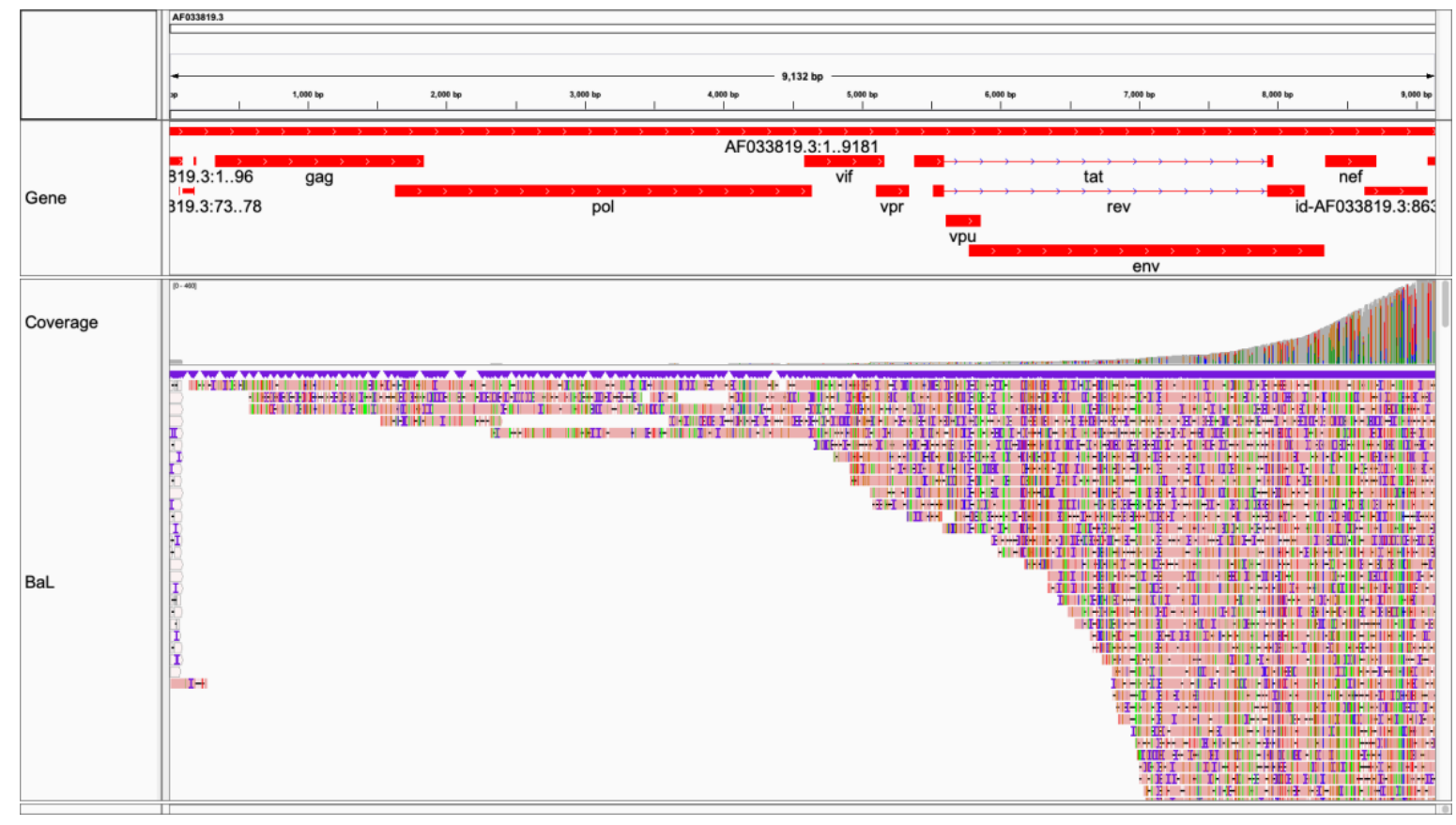

Majority coverage $\left(8,597 / 9,181^{\sim} 93.6 \%\right)$ achieved. Visualized in Integrative Genomics

Viewer [49]. Coverage over env sufficient for cladistics (Figure 4). 
Figure $2 \mathrm{H}$ : ELI

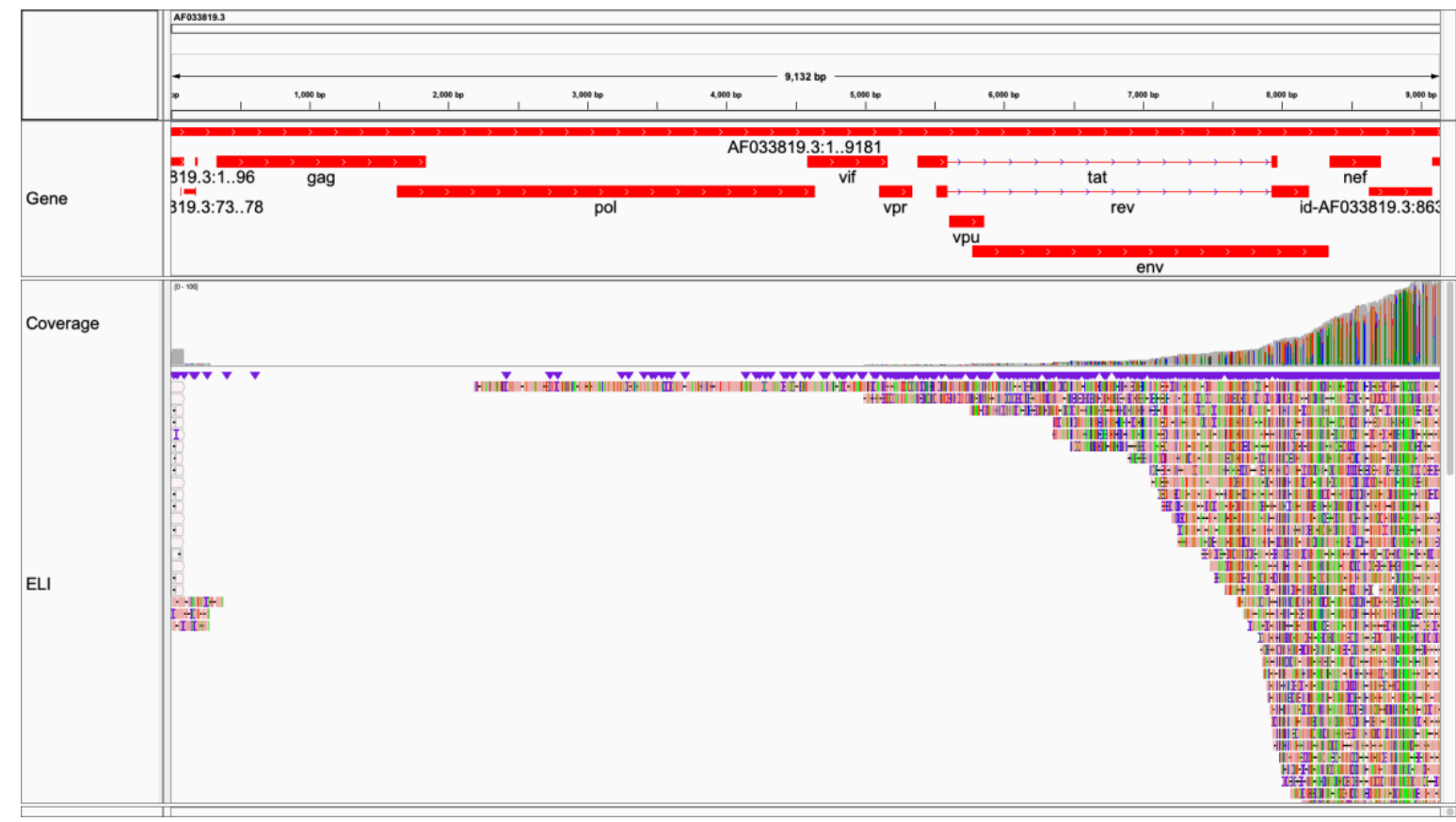

Majority coverage $(6,987 / 9,181 \sim 76.1 \%)$ achieved. Note slight change in slope over RRE.

Visualized in Integrative Genomics Viewer [49]. Coverage over env possibly sufficient for cladistics (Figure 4). 
Figure 2I: HIV1-SX

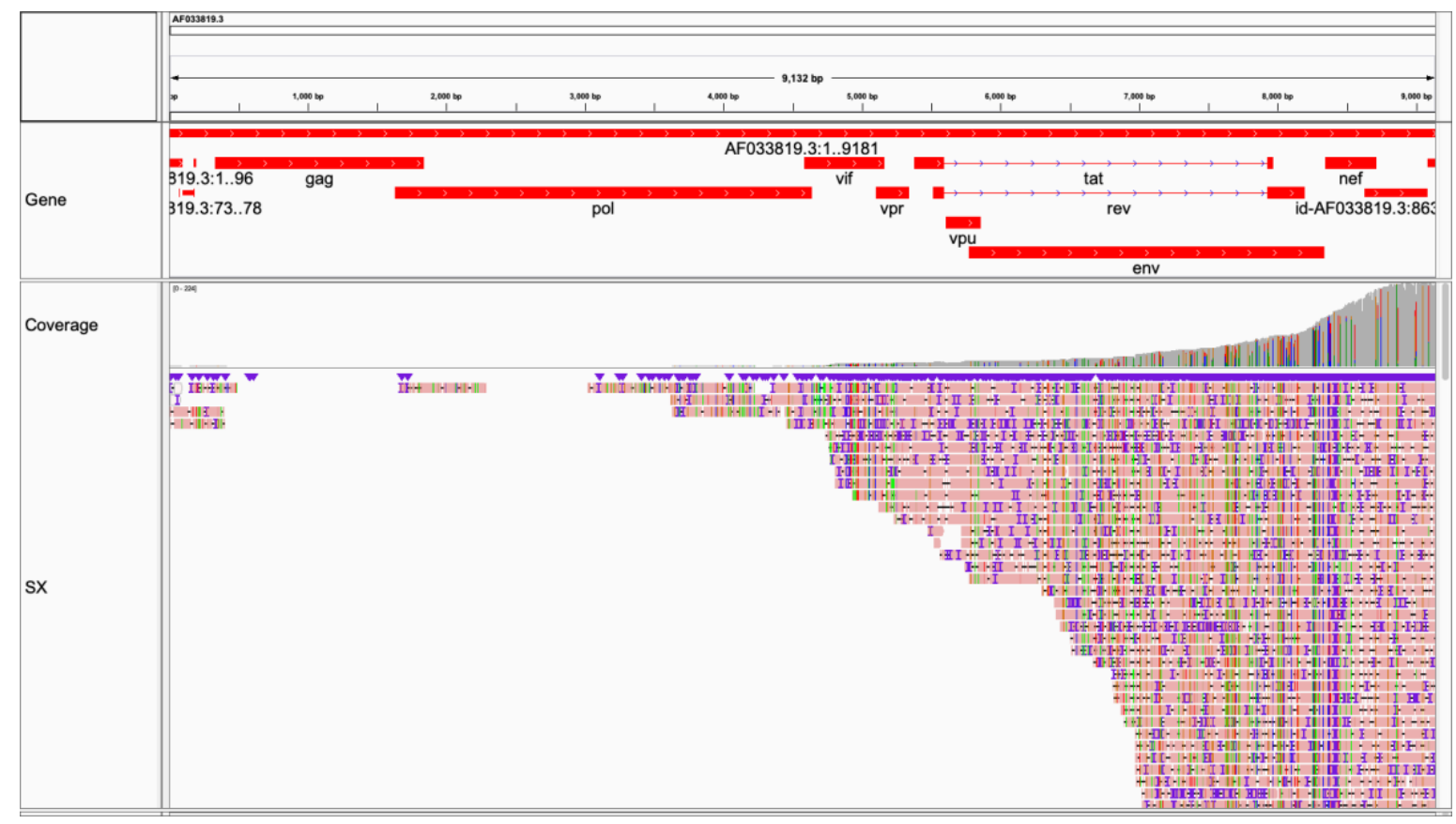

Majority coverage (5,561/9,181 60.6\%) achieved. Compare HIV1-SX, NLAD8, pNL4-3.

Visualized in Integrative Genomics Viewer [49]. Coverage over env sufficient for cladistics

(Figure 4). 
Figure 2J: JR-FL

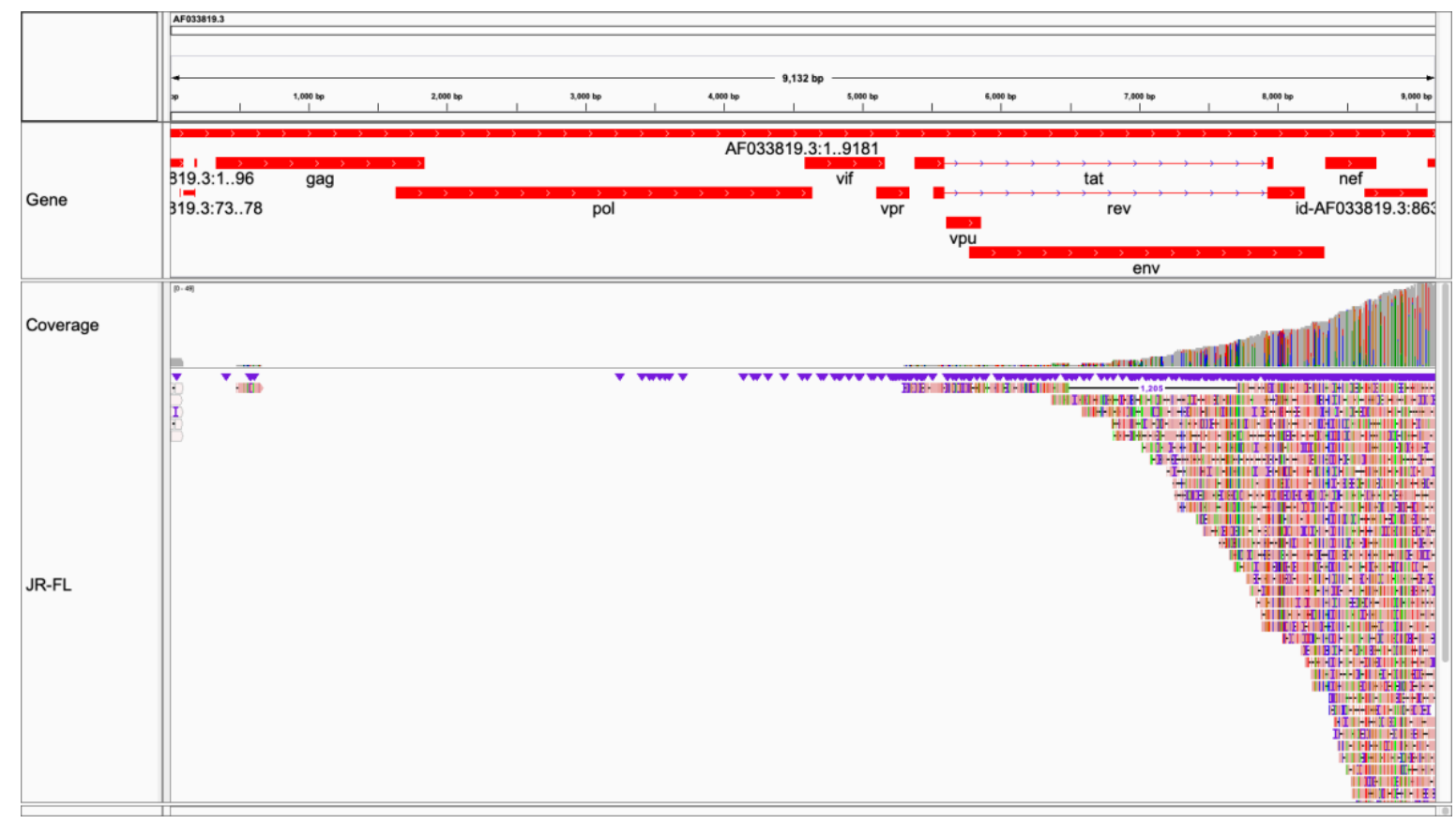

Moderate coverage $(2,814 / 9,181 \sim 30.7 \%)$ achieved. Visualized in Integrative Genomics

Viewer [49]. Coverage over env insufficient for cladistics. 
Figure 2K: MN

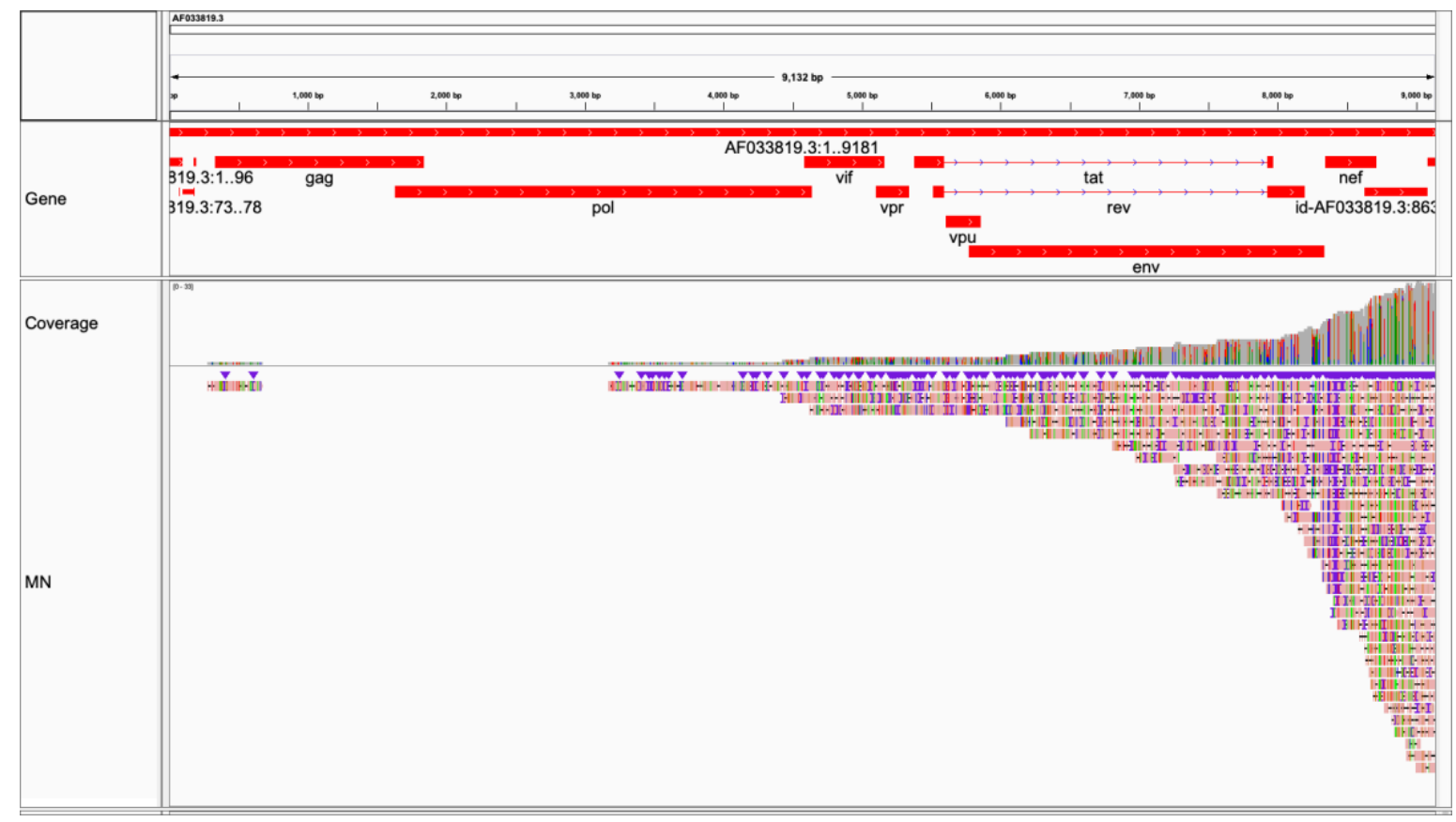

Majority coverage $(5,995 / 9,181 \sim 65.3 \%)$ achieved. Visualized in Integrative Genomics

Viewer [49]. Coverage over env possibly sufficient for cladistics (Figure 4). 
Figure 2L: NL4-3

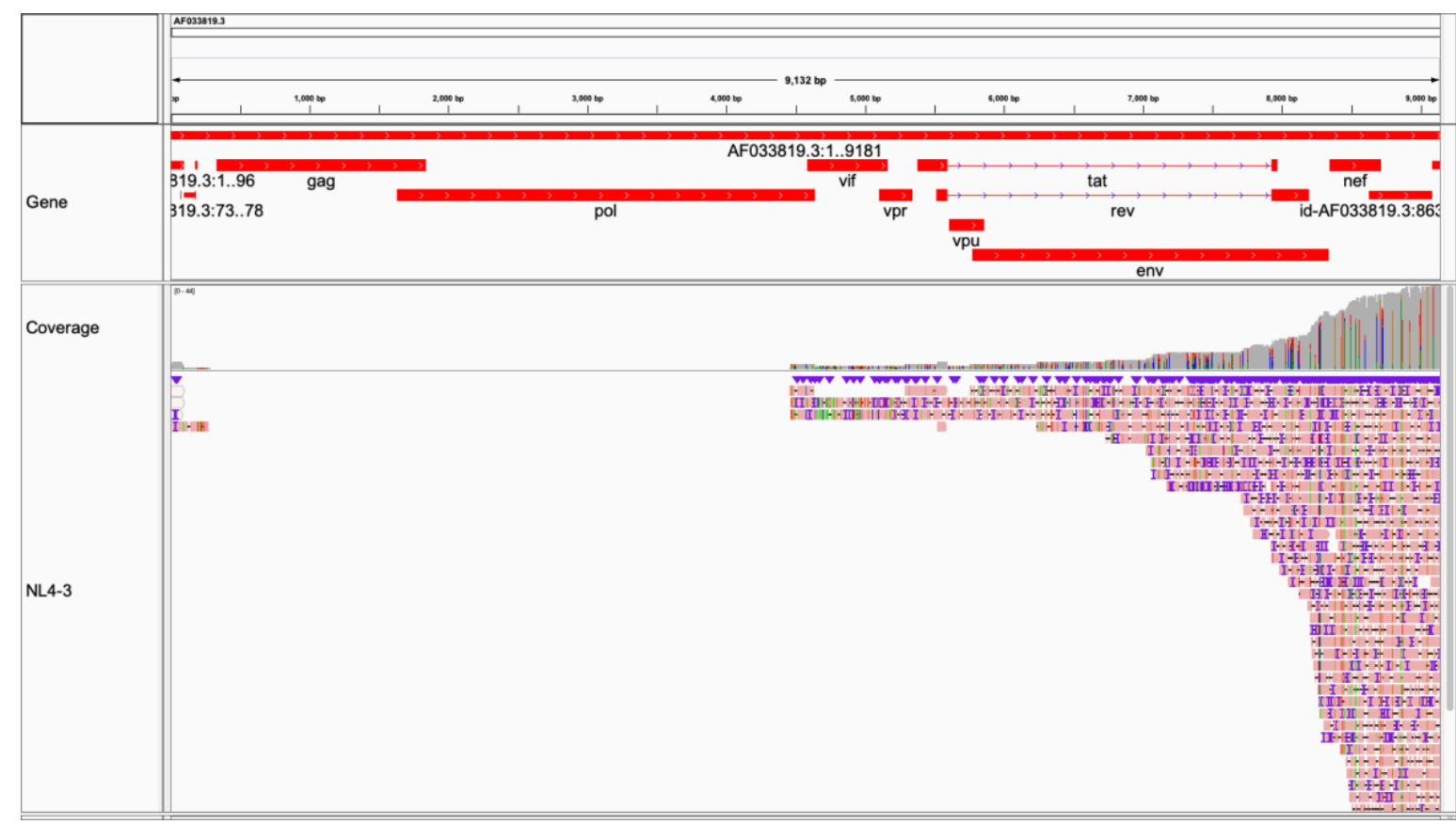

Majority coverage $(4,715 / 9,181 \sim 51.4 \%)$ achieved. Note slight change in slope over RRE.

Visualized in Integrative Genomics Viewer [49]. Coverage over env possibly sufficient for cladistics (Figure 4). 
Figure 2M: NLAD8

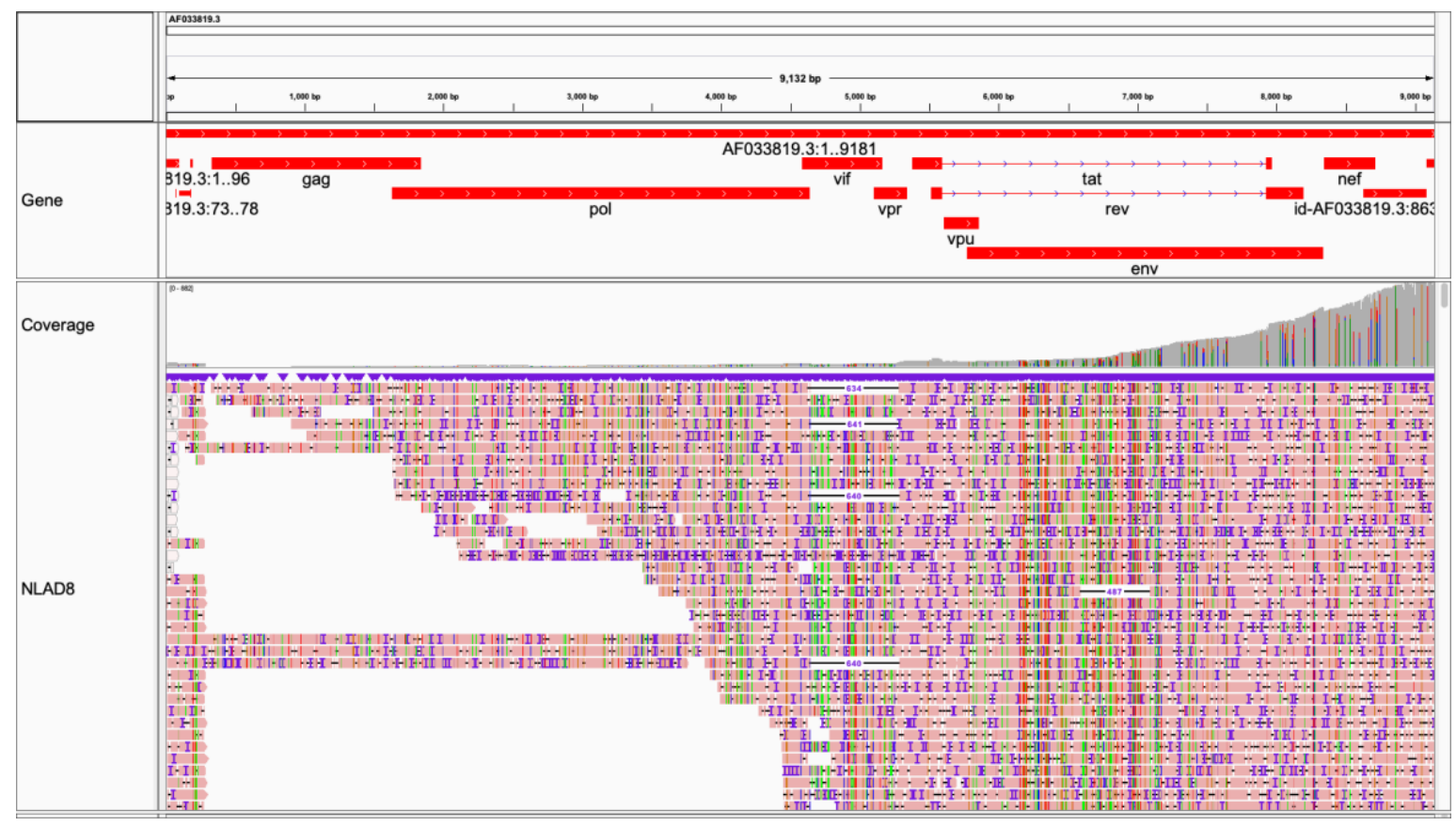

Full-length coverage $(9,166 / 9,181 \sim 99.8 \%)$ achieved. Visualized in Integrative Genomics

Viewer [49]. Coverage over env sufficient for cladistics (Figure 4). 
Figure 2N: RHPA

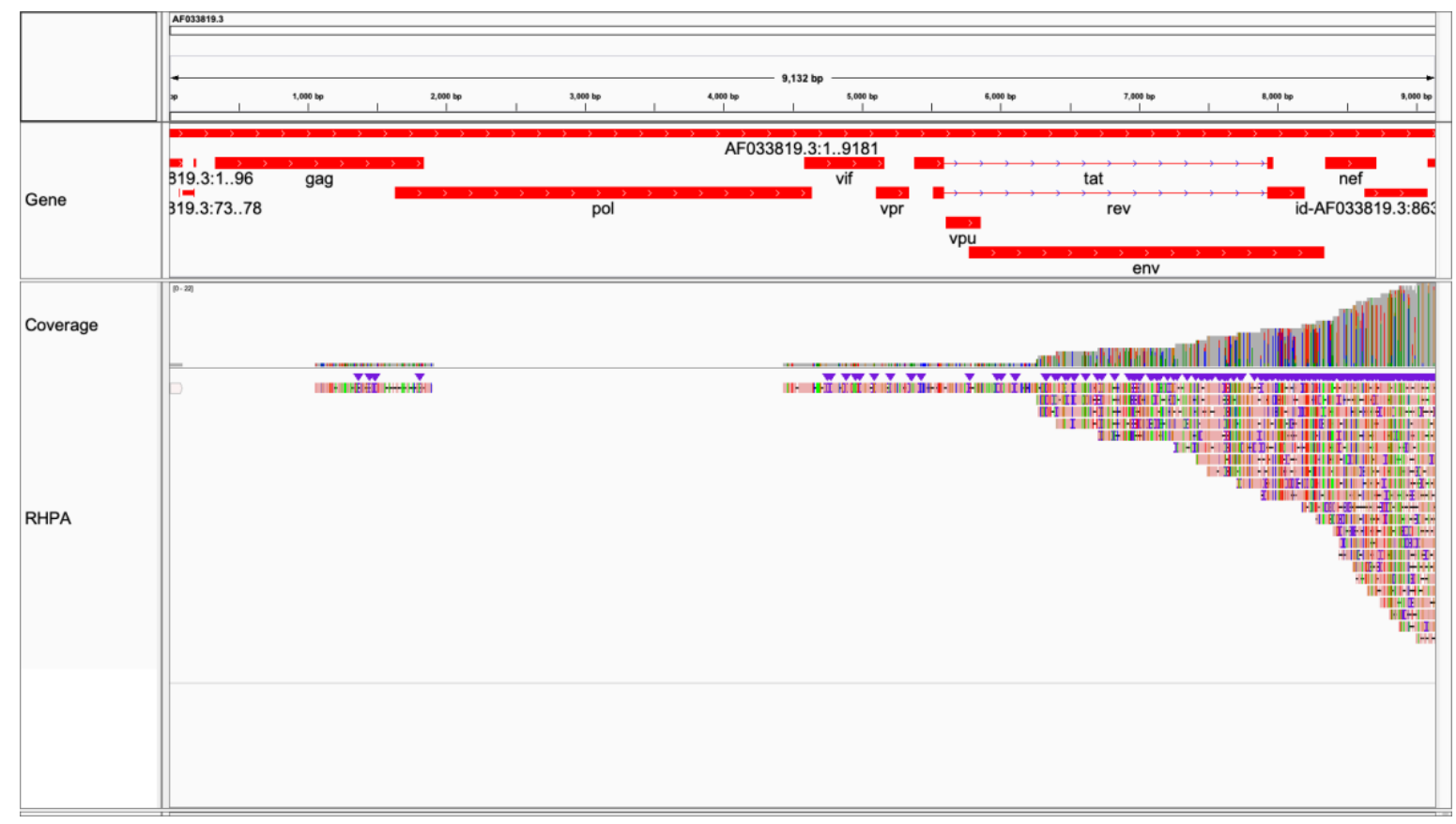

Majority coverage $(4,745 / 9,181 \sim 51.7 \%)$ achieved. Visualized in Integrative Genomics

Viewer [49]. Coverage over env insufficient for cladistics. 
Figure 20: SF162

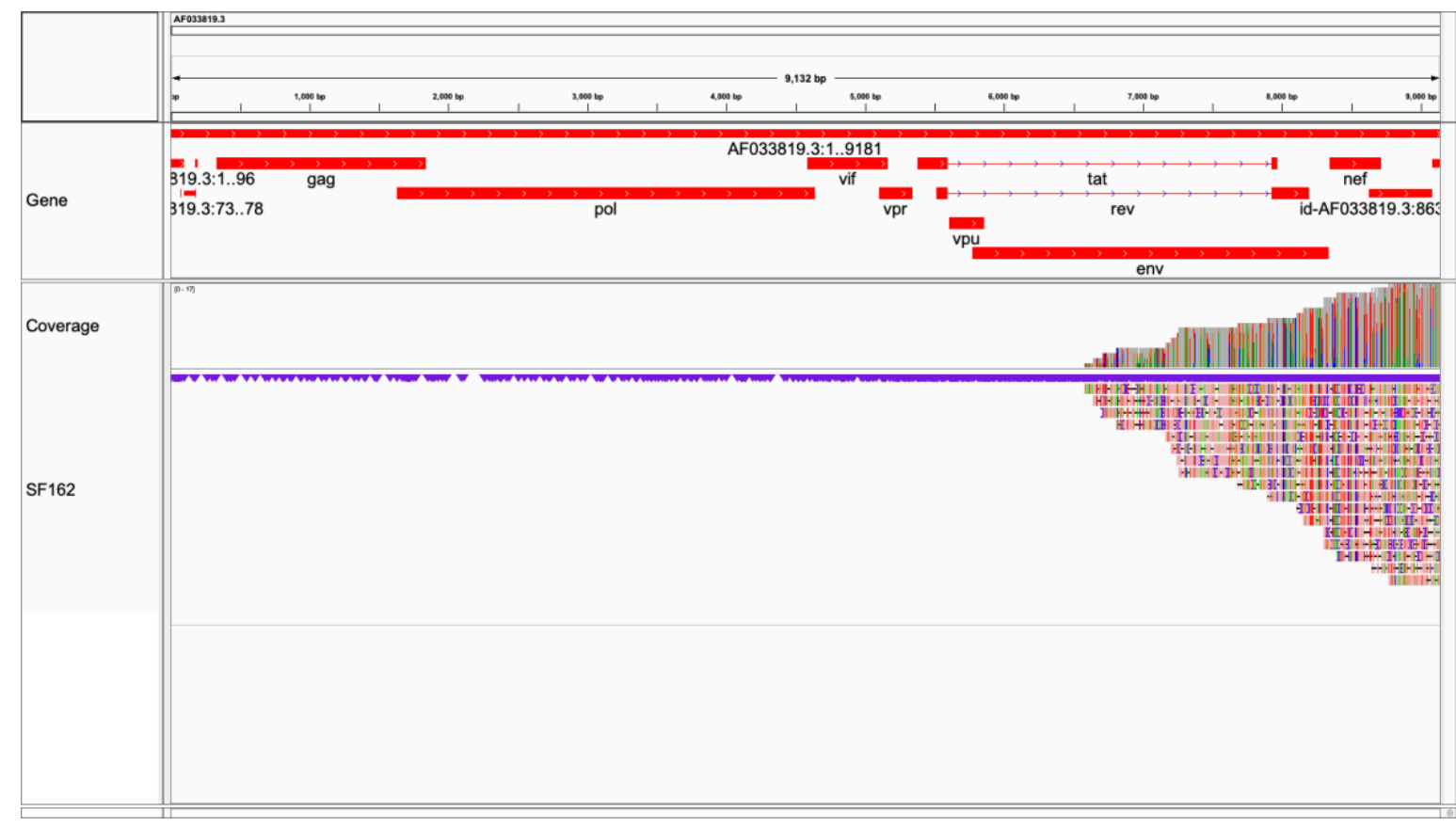

Moderate coverage $(2,601 / 9,181 \sim 28.3 \%)$ achieved. Note slight change in slope over RRE.

Visualized in Integrative Genomics Viewer [49]. Coverage over env insufficient for

cladistics. 
Figure 3A: 89.6 mapped to 89.6

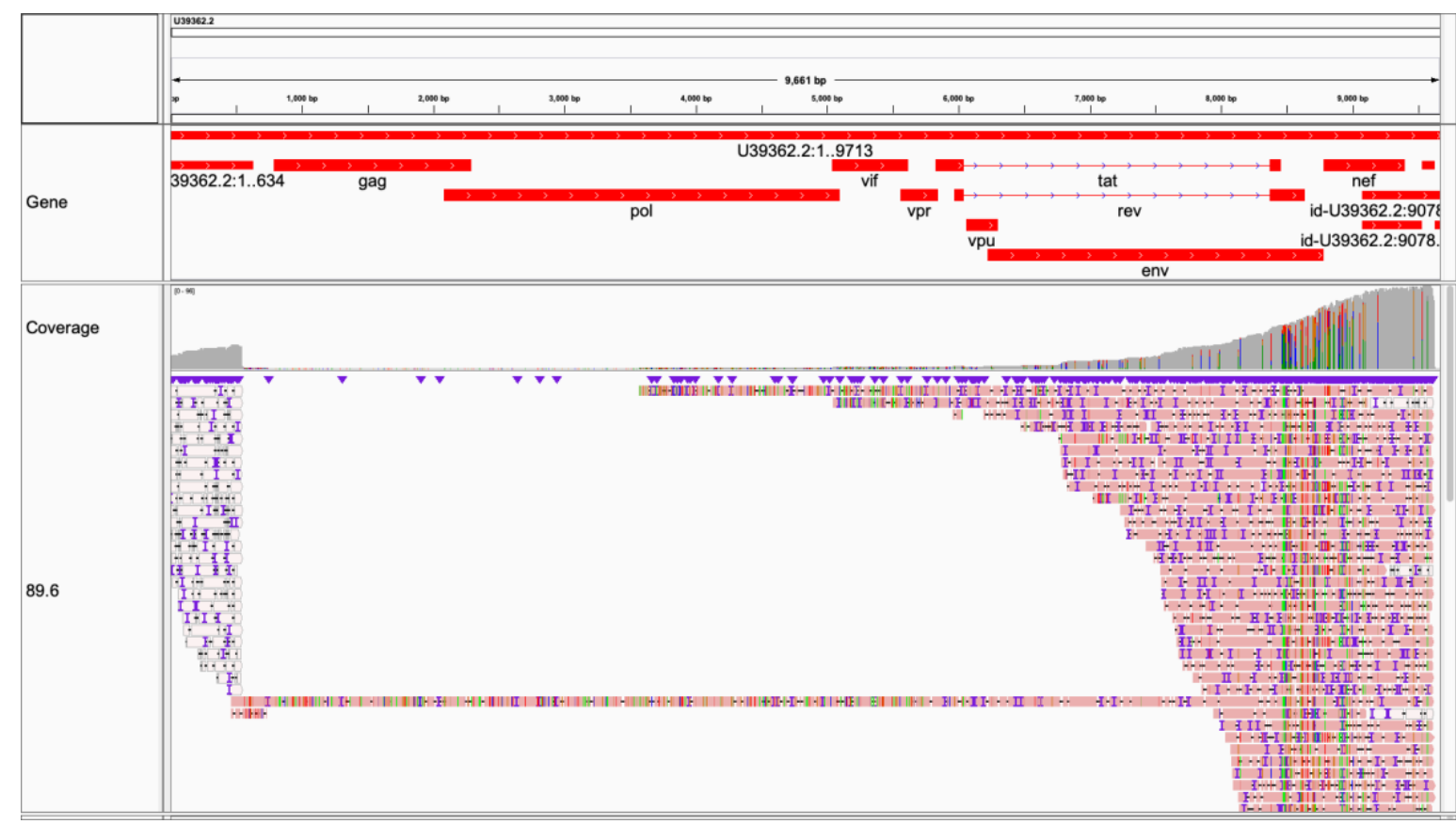

Strain-specific reference mapping. Gray in coverage plot (and pink in read body) indicates

per-base consensus accuracy $\geq 80 \%$. Visualized in Integrative Genomics Viewer [49]. 
Figure 3B: 92 UG029 mapped to 92UG029

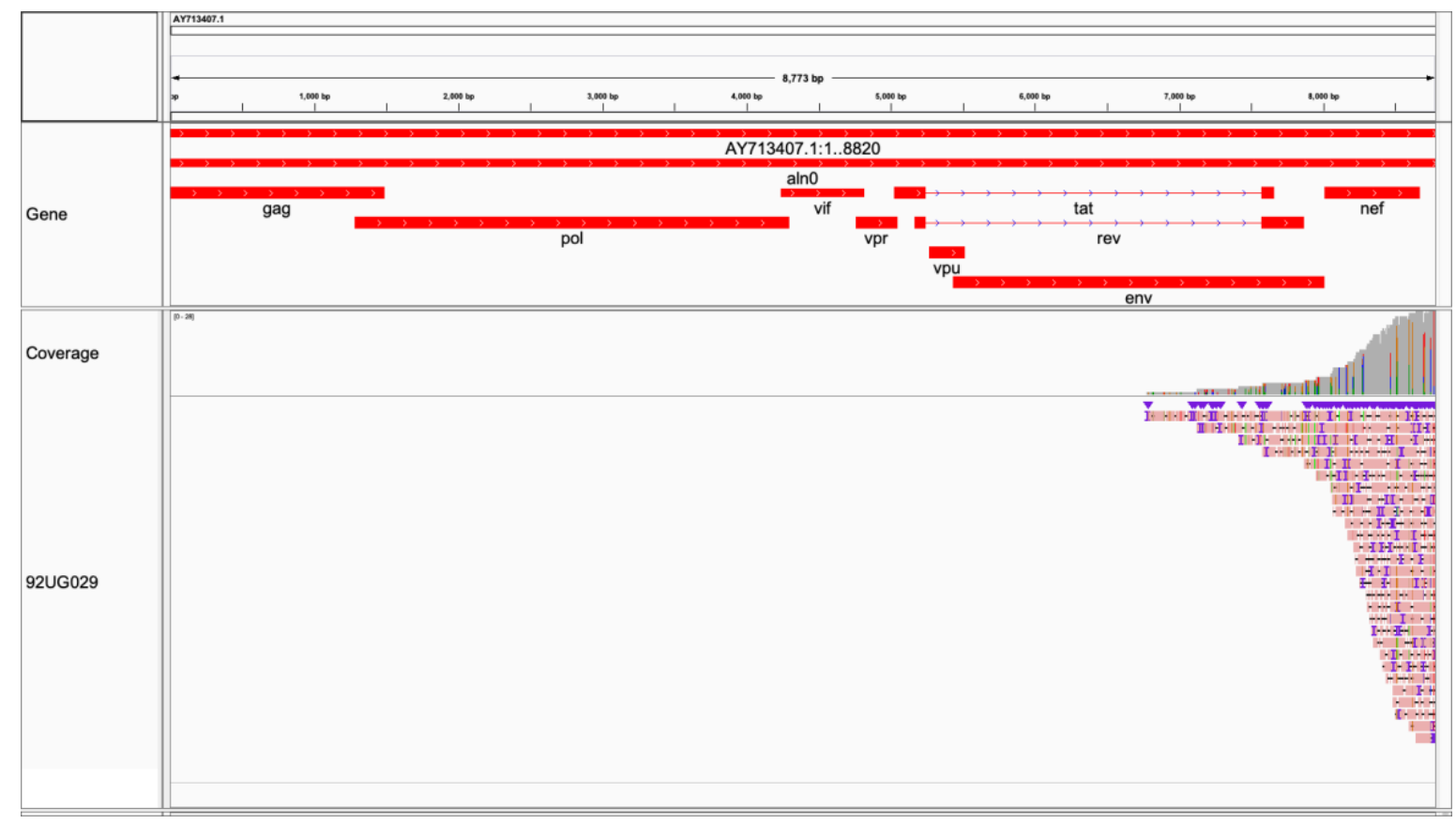

Strain-specific reference mapping. Gray in coverage plot (and pink in read body) indicates

per-base consensus accuracy $\geq 80 \%$. Visualized in Integrative Genomics Viewer [49]. 
Figure 3C: BaL mapped to BaL

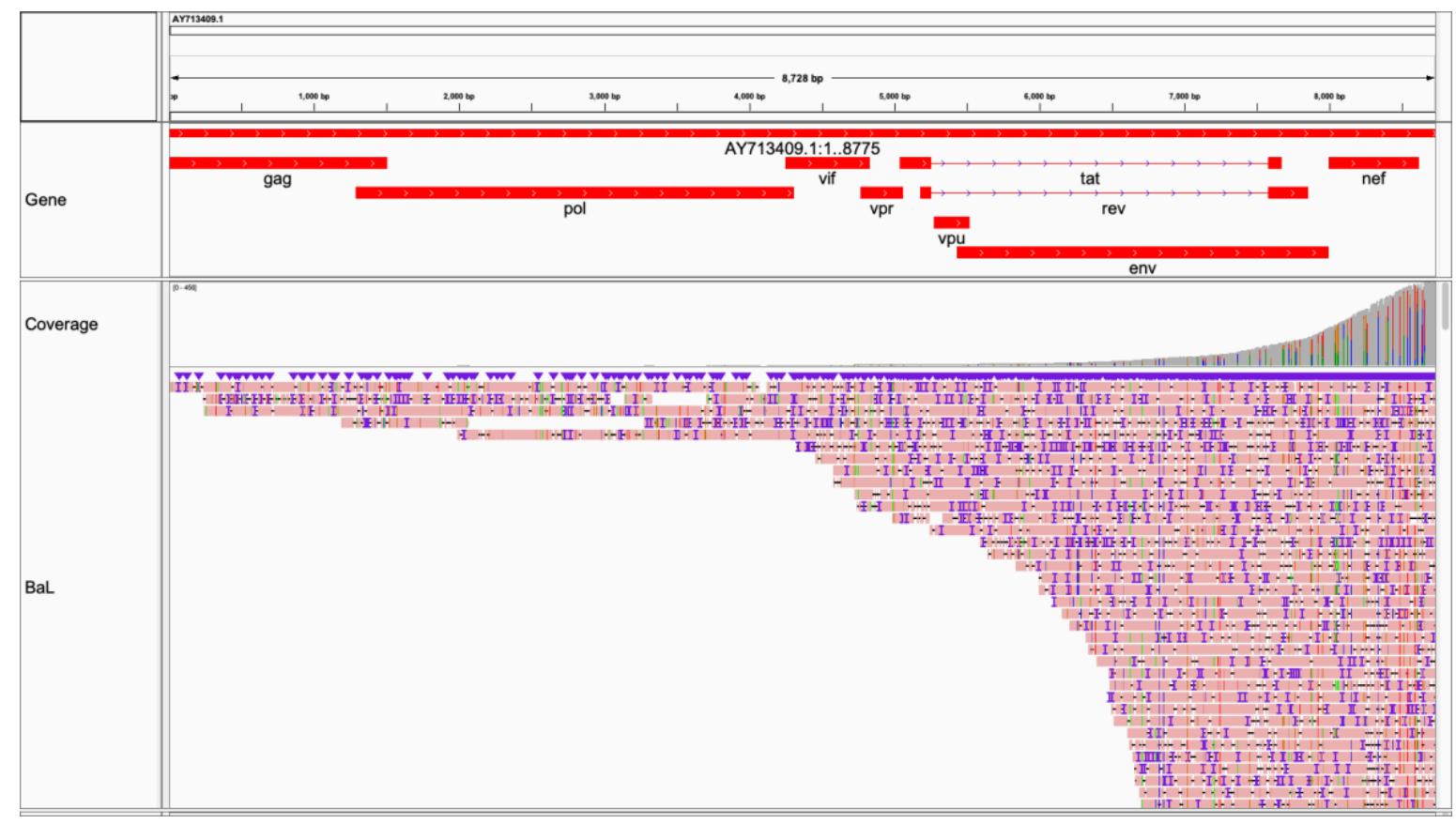

Strain-specific reference mapping. Gray in coverage plot (and pink in read body) indicates per-base consensus accuracy $\geq 80 \%$. Note BaL reference does not include LTRs. Visualized in Integrative Genomics Viewer [49]. 
Figure 3D: ELI mapped to ELI

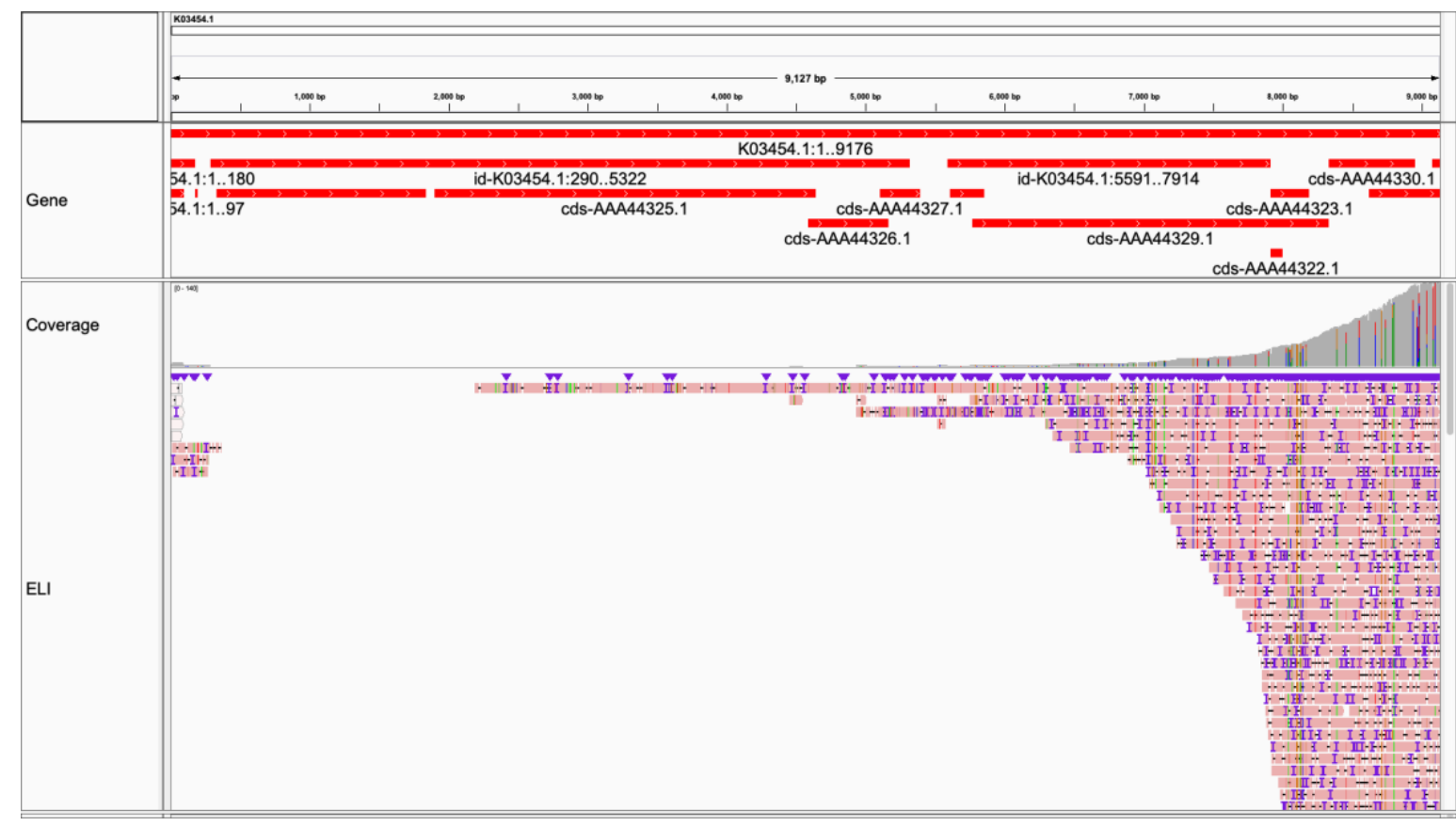

Strain-specific reference mapping. Gray in coverage plot (and pink in read body) indicates

per-base consensus accuracy $\geq 80 \%$. Note slight change in slope over RRE. Visualized in Integrative Genomics Viewer [49]. 
Figure 3E: HIV1-SX mapped to pNL4-3

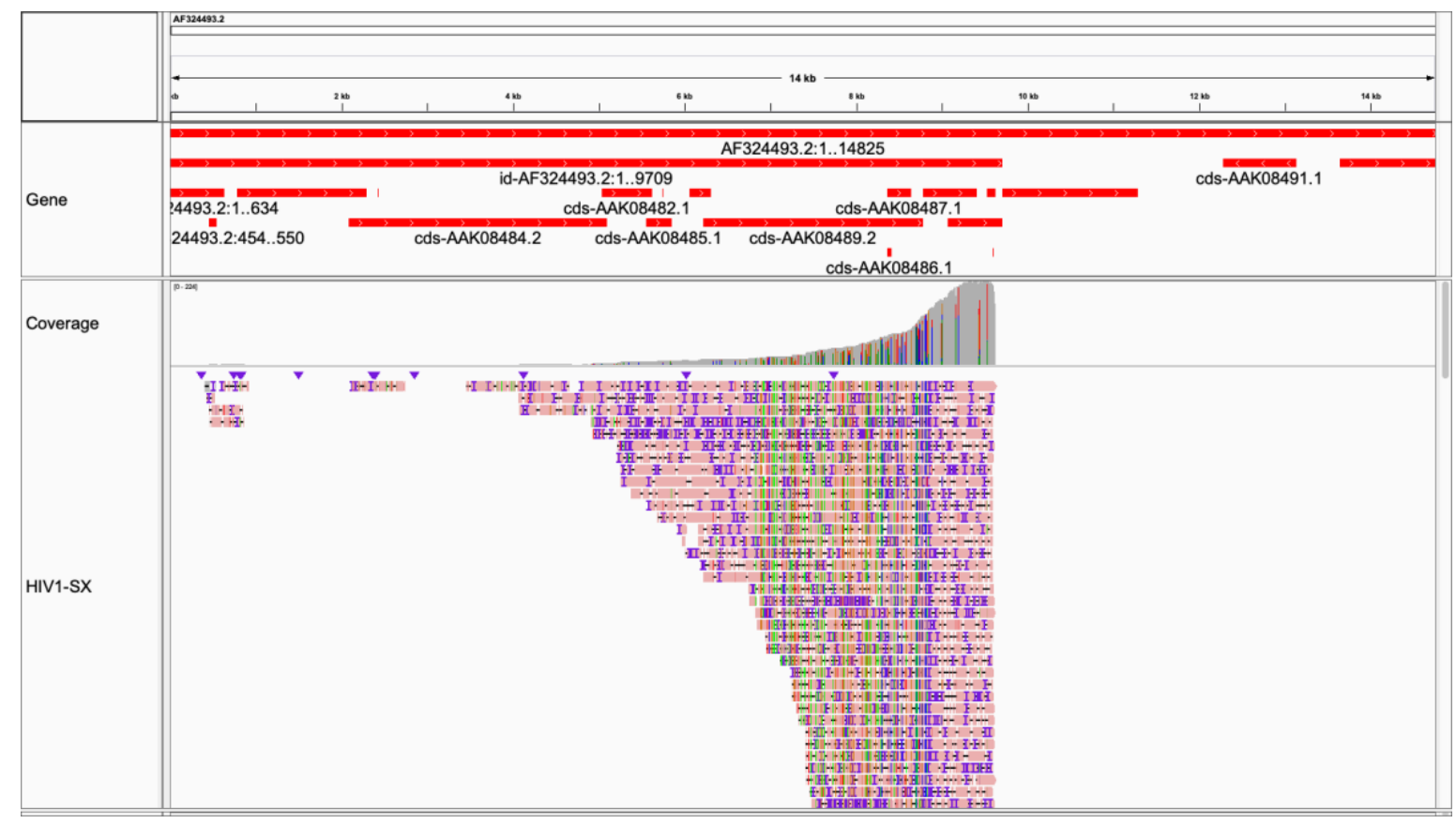

Strain-specific reference mapping. Gray in coverage plot (and pink in read body) indicates

per-base consensus accuracy $\geq 80 \%$. Visualized in Integrative Genomics Viewer [49]. 
Figure 3F: MN mapped to MN

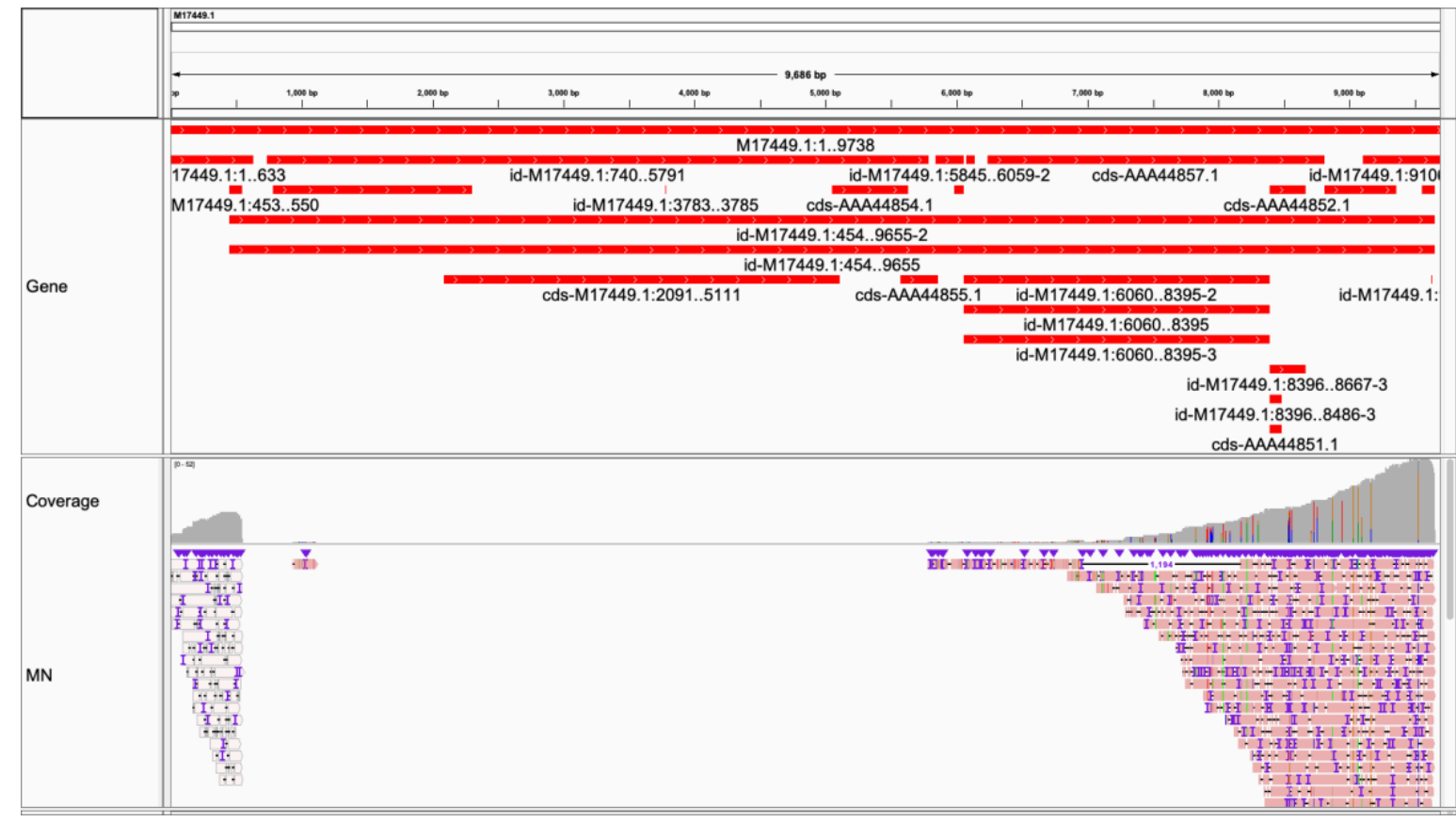

Strain-specific reference mapping. Gray in coverage plot (and pink in read body) indicates

per-base consensus accuracy $\geq 80 \%$. Visualized in Integrative Genomics Viewer [49]. 
Figure 3G: NL4-3 mapped to pNL4-3

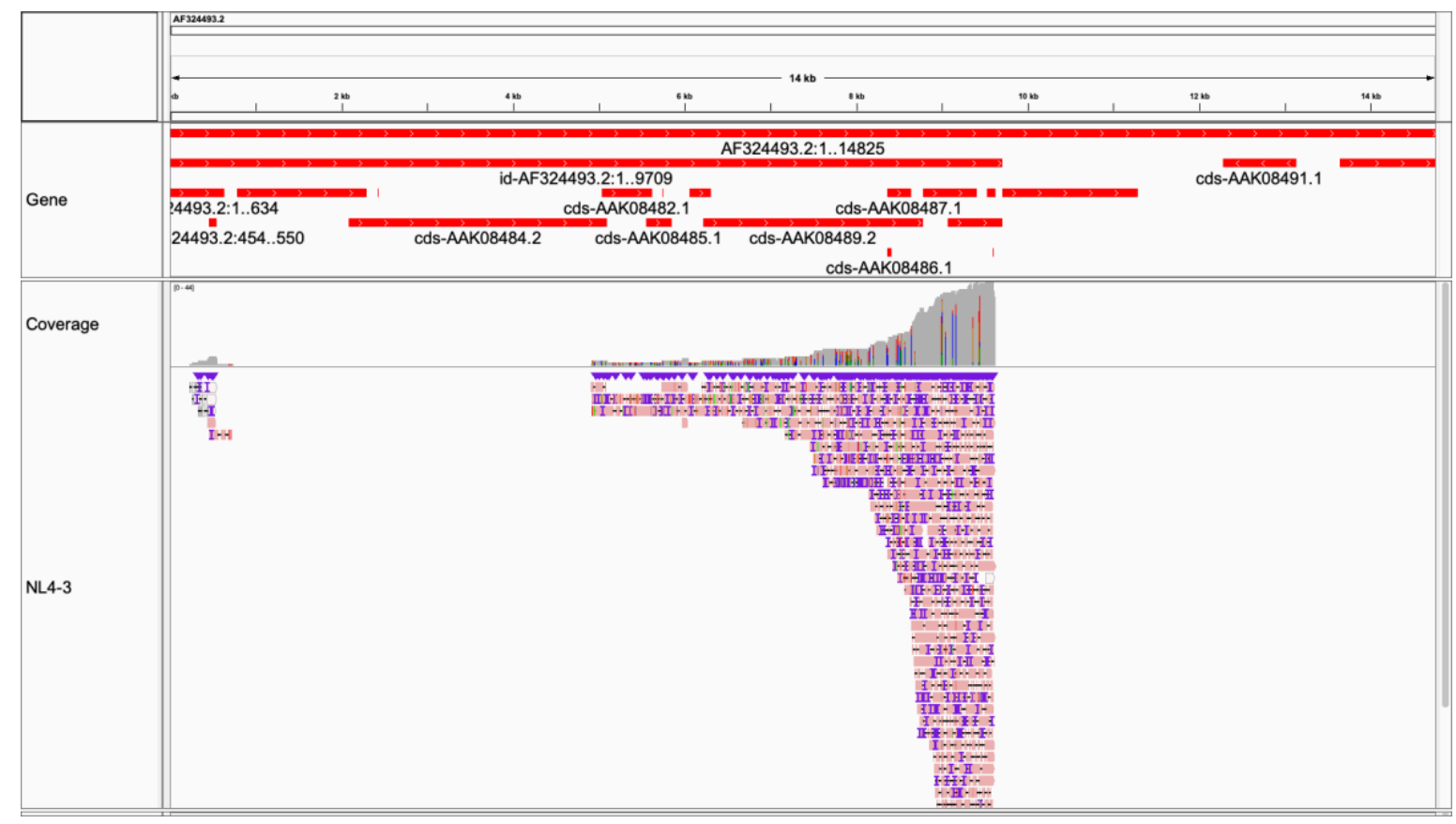

Gray in coverage plot (and pink in read body) indicates per-base consensus accuracy $\geq$

$80 \%$. Note slight change in slope over RRE. Visualized in Integrative Genomics Viewer

[49]. 
Figure 3H, 3I: NLAD8 mapped to AD8, pNL4-3
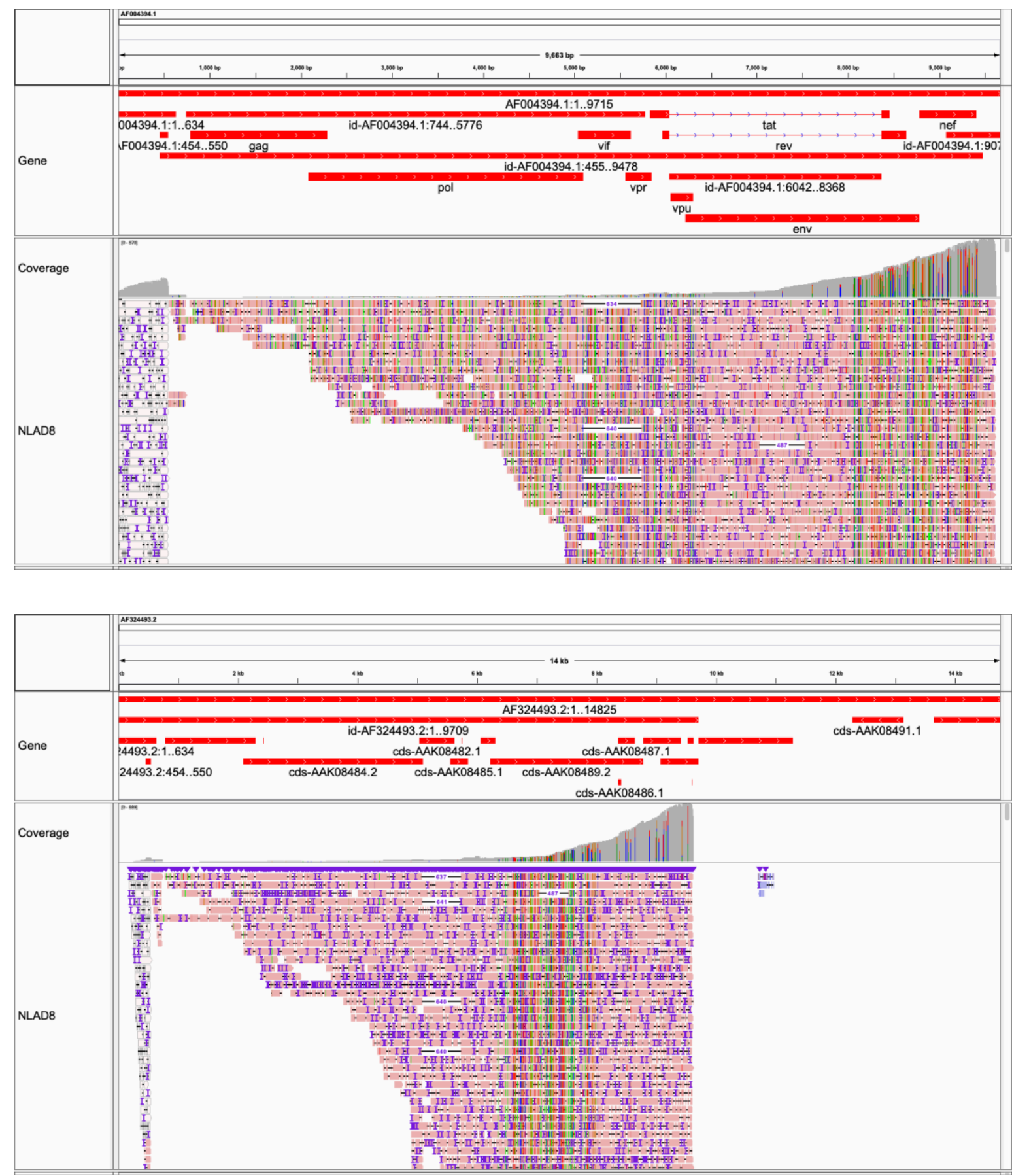

Strain-specific reference mapping. Gray in coverage plot (and pink in read body) indicates

per-base consensus accuracy $\geq 80 \%$. Note that NLAD8 is a chimera between NL4-3 (all

minus portion of env) and AD8 (portion of env) (used as a reference here). The reference

sequence for NL4-3 is a plasmid, pNL4-3. NLAD8 complete genome was not available.

Note the difference in scale. Visualized in Integrative Genomics Viewer [49]. 
Figure 3J: RHPA mapped to RHPA

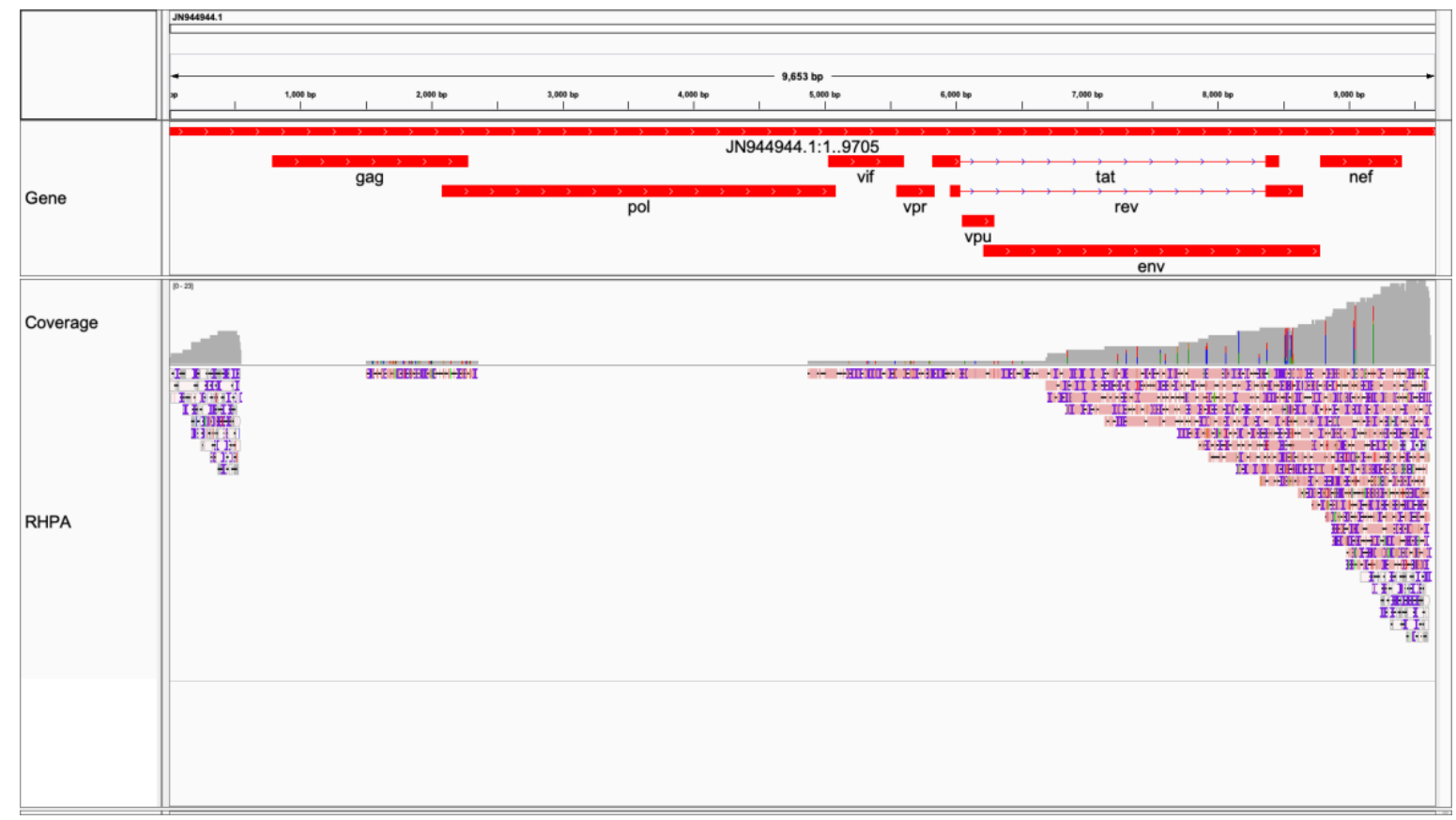

Strain-specific reference mapping. Gray in coverage plot (and pink in read body) indicates

per-base consensus accuracy $\geq 80 \%$. Visualized in Integrative Genomics Viewer [49]. 
bioRxiv preprint doi: https://doi.org/10.1101/845610; this version posted November 19, 2019. The copyright holder for this preprint (which was not certified by peer review) is the author/funder. All rights reserved. No reuse allowed without permission.

54

Figure 4: ONT native RNA + Canu de novo assembly sufficient for comparative

transcriptomics.

A

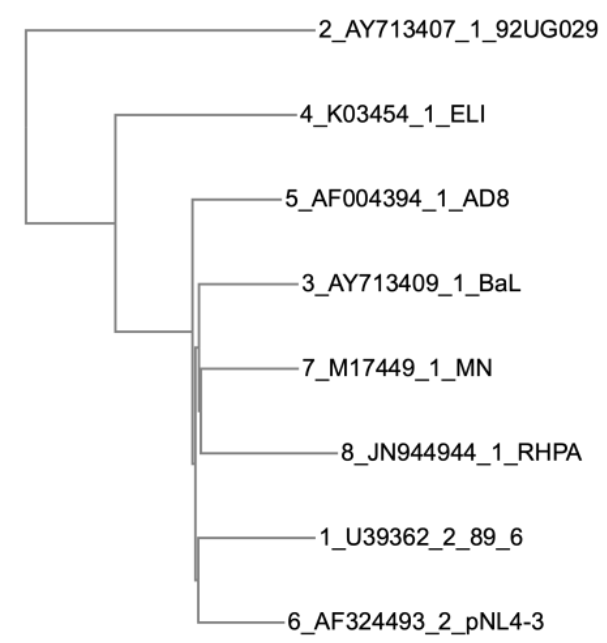

B

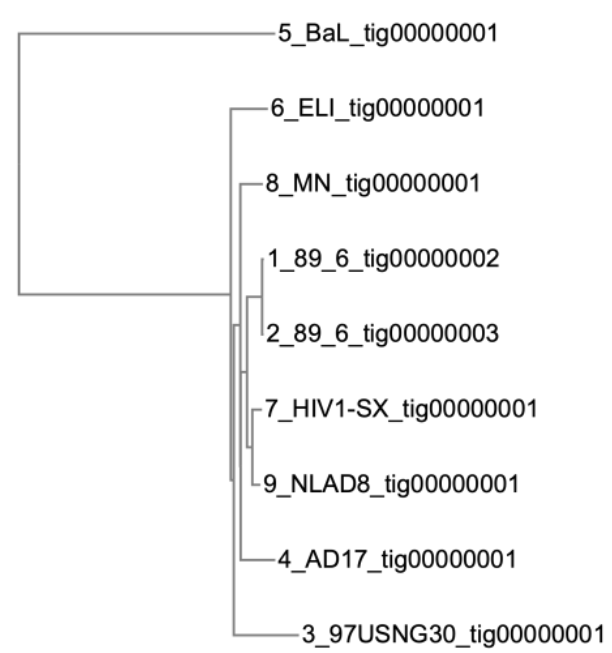


bioRxiv preprint doi: https://doi.org/10.1101/845610; this version posted November 19,2019 . The copyright holder for this preprint (which was not certified by peer review) is the author/funder. All rights reserved. No reuse allowed without permission.

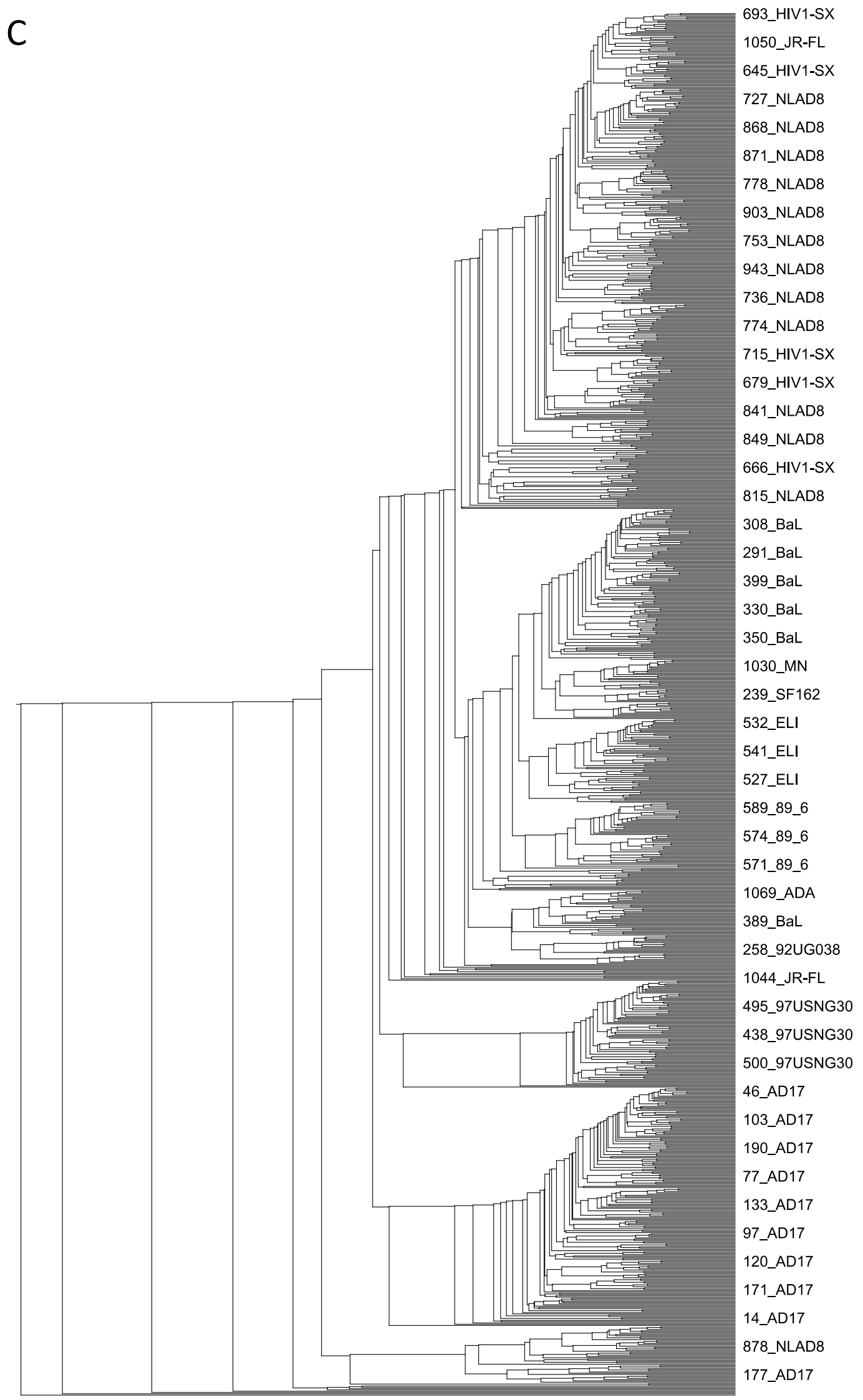


Trees from multisequence alignments. Phylogenetic relationships (subtype, relationship to NL4-3) evident in trees from full reference genomes are generally conserved at the contig and individual sequence levels. A. Available full-length genomes (left). B. Contigs (right). C. All recovered HIV reads. MAFFT [15]. 
bioRxiv preprint doi: https://doi.org/10.1101/845610; this version posted November 19, 2019. The copyright holder for this preprint (which was not certified by peer review) is the author/funder. All rights reserved. No reuse allowed without permission.

SUPPLEMENTAL DATA 
[Insert here]

Supplemental Table 1: Library Statistics

\begin{tabular}{|c|c|c|c|c|c|c|}
\hline Sample & $\begin{array}{l}\text { Read } \\
\text { count }\end{array}$ & $\begin{array}{c}\text { Average } \\
\text { length }\end{array}$ & $\begin{array}{c}\text { Median } \\
\text { length }\end{array}$ & $\begin{array}{c}\text { Max } \\
\text { length }\end{array}$ & $\begin{array}{c}\text { Avg mean } \\
\text { QS }\end{array}$ & $\begin{array}{c}\text { Median mean } \\
\text { QS }\end{array}$ \\
\hline 89.6 & 50 & 1258 & 922 & 8679 & 8.31 & 8.56 \\
\hline 92UG029_run1 & 4 & 1161 & 1116 & 1731 & 10.42 & 10.45 \\
\hline 92UG029_run2 & 2 & 937 & 937 & 989 & 9.08 & 9.08 \\
\hline 92UG029_run3 & 2 & 1180 & 1180 & 2023 & 9.66 & 9.66 \\
\hline 92 UG038 & 4 & 1159 & 1240 & 1401 & 10.19 & 9.76 \\
\hline 92UG038_skip & 8 & 1622 & 1490 & 3678 & 10.63 & 10.71 \\
\hline 97USNG30 & 22 & 1333 & 1151 & 3742 & 10.56 & 10.48 \\
\hline 97USNG30_skip & 61 & 1292 & 1007 & 4400 & 10.96 & 11.09 \\
\hline AD17_run1 & 42 & 2026 & 1508 & 7176 & 10.08 & 10.12 \\
\hline AD17_run1_skip & 109 & 1760 & 1343 & 5508 & 10.30 & 10.48 \\
\hline AD17_run2 & 31 & 2303 & 1700 & 7390 & 9.79 & 10.03 \\
\hline AD17_run2_skip & 12 & 2249 & 1256 & 8830 & 10.52 & 10.71 \\
\hline ADA_run1 & 5 & 617 & 565 & 845 & 9.79 & 9.77 \\
\hline ADA_run2 & 1 & 1334 & 1334 & 1334 & 11.14 & 11.14 \\
\hline ADA_run3 & 4 & 852 & 616 & 1904 & 9.58 & 9.90 \\
\hline ADA_run4 & 0 & NA & NA & NA & NA & NA \\
\hline $\mathrm{BaL}$ & 22 & 969 & 905 & 2038 & 9.79 & 9.81 \\
\hline BaL_skip & 147 & 1020 & 765 & 4474 & 10.08 & 10.26 \\
\hline ELI_run1 & 9 & 2017 & 809 & 6909 & 10.08 & 10.11 \\
\hline ELI_run1_skip & 30 & 905 & 763 & 2887 & 9.72 & 10.01 \\
\hline ELI_run2 & 5 & 1017 & 989 & 1921 & 11.05 & 11.62 \\
\hline ELI_run2_skip & 7 & 890 & 531 & 1892 & 9.98 & 10.03 \\
\hline HIV1-SX & 107 & 1278 & 954 & 4796 & 10.02 & 10.10 \\
\hline$J R-F L$ & 21 & 1345 & 783 & 4695 & 9.98 & 9.75 \\
\hline $\mathrm{MN}$ & 25 & 846 & 638 & 1912 & 10.12 & 10.41 \\
\hline NL4-3_run3 & 19 & 1211 & 948 & 4626 & 9.92 & 10.23 \\
\hline NL4-3_run5 & 9 & 1475 & 1021 & 4630 & 9.04 & 8.80 \\
\hline NLAD8 & 291 & 1577 & 1081 & 8956 & 9.97 & 10.16 \\
\hline RHPA & 13 & 1447 & 890 & 4696 & 10.39 & 10.90 \\
\hline SF162 & 6 & 1849 & 1950 & 2540 & 10.60 & 11.34 \\
\hline SF162_skip & 5 & 1041 & 862 & 2020 & 10.48 & 10.20 \\
\hline
\end{tabular}


Supplemental Figure 1: Consistent mapping and read quality between biological replicates.

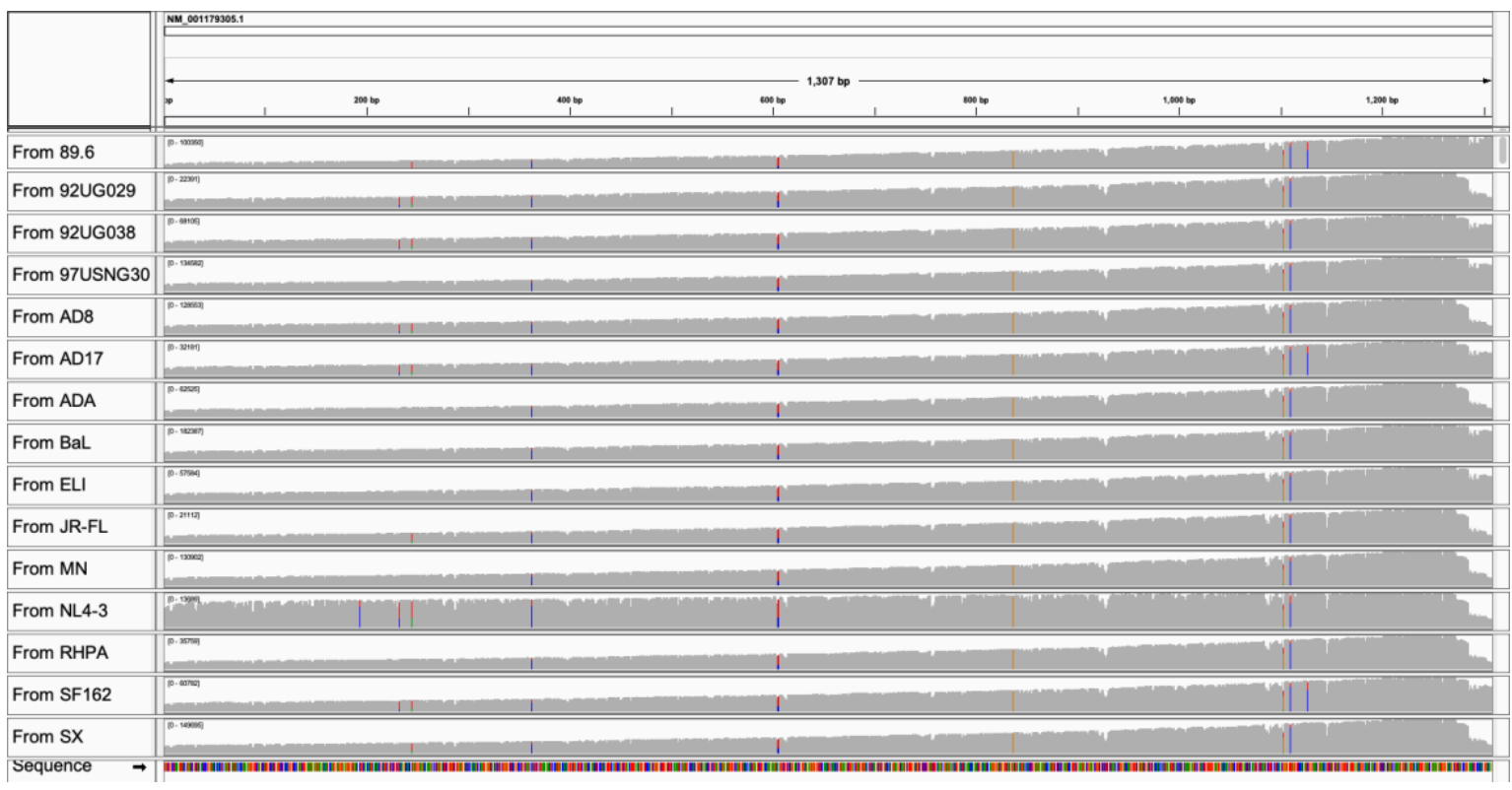

NL4-3 was processed with a previously used R9.4 RevC flow cell (now discontinued) in a prior pilot study. All other samples processed with R9.4.1 RevD flow cells. See Methods section for more details about control RNA. Alignments with minimap2 [13] in usegalaxy.eu [14]. Visualized in Integrative Genomics Viewer [49]. 
Supplemental Table 2: For SQK-RNA002, coverage drops by approximately 2/3rds

per 1,000 bases.

\section{Coverage @}

\begin{tabular}{|l|c|c|c|c|r|}
\hline \multicolumn{1}{|c|}{ Sample } & base 1,200 & base 200 & \multicolumn{1}{c|}{$\boldsymbol{\Delta}$} & slope & $\begin{array}{c}\text { \% decrease } \\
\text { per 1,000 bases }\end{array}$ \\
\hline 89.6 & 92,073 & 28,252 & $-63,821$ & -63.82 & 69.32 \\
92UG029 & 20,734 & 6,580 & $-14,154$ & -14.15 & 68.26 \\
92UG038 & 62,967 & 20,566 & $-42,401$ & -42.40 & 67.34 \\
97USNG30 & 122,401 & 38,524 & $-83,877$ & -83.88 & 68.53 \\
AD8 & 119,191 & 37,522 & $-81,669$ & -81.67 & 68.52 \\
AD17 & 29,862 & 9,610 & $-20,252$ & -20.25 & 67.82 \\
ADA & 57,494 & 17,422 & $-40,072$ & -40.07 & 69.70 \\
BaL & 166,932 & 52,636 & $-114,296$ & -114.30 & 68.47 \\
ELI & 53,150 & 16,865 & $-36,285$ & -36.29 & 68.27 \\
JR-FL & 19,534 & 6,102 & $-13,432$ & -13.43 & 68.76 \\
MN & 120,389 & 37,791 & $-82,598$ & -82.60 & 68.61 \\
NL4-3 & 13,485 & 10,039 & $-3,446$ & -3.45 & 25.55 \\
RHPA & 32,874 & 10,320 & $-22,554$ & -22.55 & 68.61 \\
SF162 & 56,309 & 18,407 & $-37,902$ & -37.90 & 67.31 \\
SX & 138,097 & 43,953 & $-94,144$ & -94.14 & 68.17 \\
Average & $\mathbf{7 3 , 6 9 9 . 4 7}$ & $\mathbf{2 3 , 6 3 9 . 2 7}$ & $-50,060.20$ & -50.06 & 65.55 \\
\hline
\end{tabular}

Data from Control RNA, which has much higher coverage compared to viral or host cell

RNA. Positions relative to reference. Counts from reads mapped to ENO2 (minimap2 in

Galaxy), visualized in Integrative Genomics Viewer [49]. 
Supplemental Figure 2: A set of homopolymer tracks from HIV-1 NLAD8.

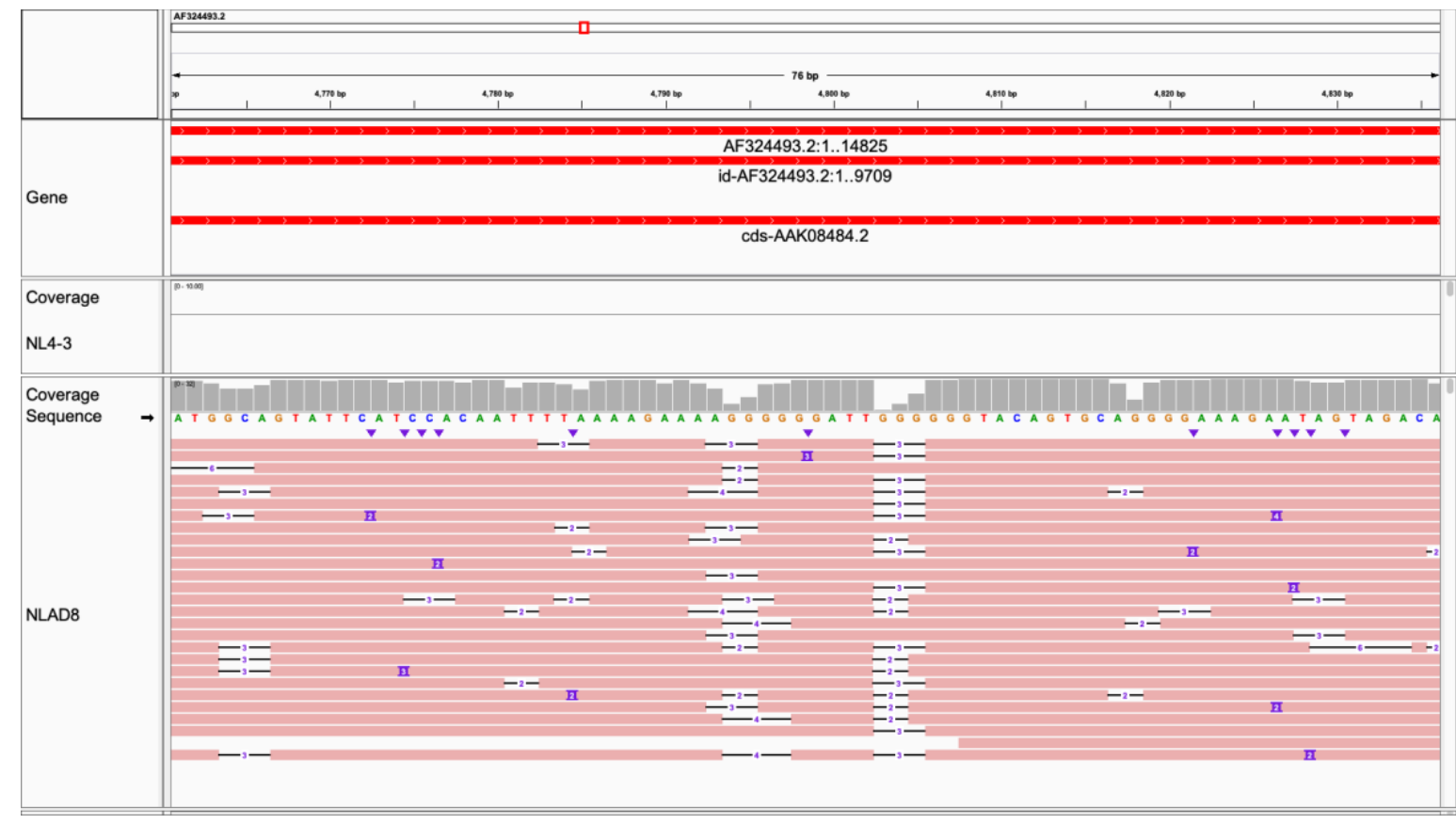

Top: AF324493.2:4,761-4,836. Alignments with minimap2 [13] in usegalaxy.eu [14].

Bottom: AF033819.3: 4,308-4,383. Note absence of insertions near trailing ends of

homopolymers, supporting mapping as an important QC step when calling variants near

these sequence features. Visualized in Integrative Genomics Viewer [49]. Comparable to

DNA ONT [3]. The native NL4-3 sample did not have coverage over this region. 
Supplemental Figure 3: SNVs near an A-homopolymer truncation
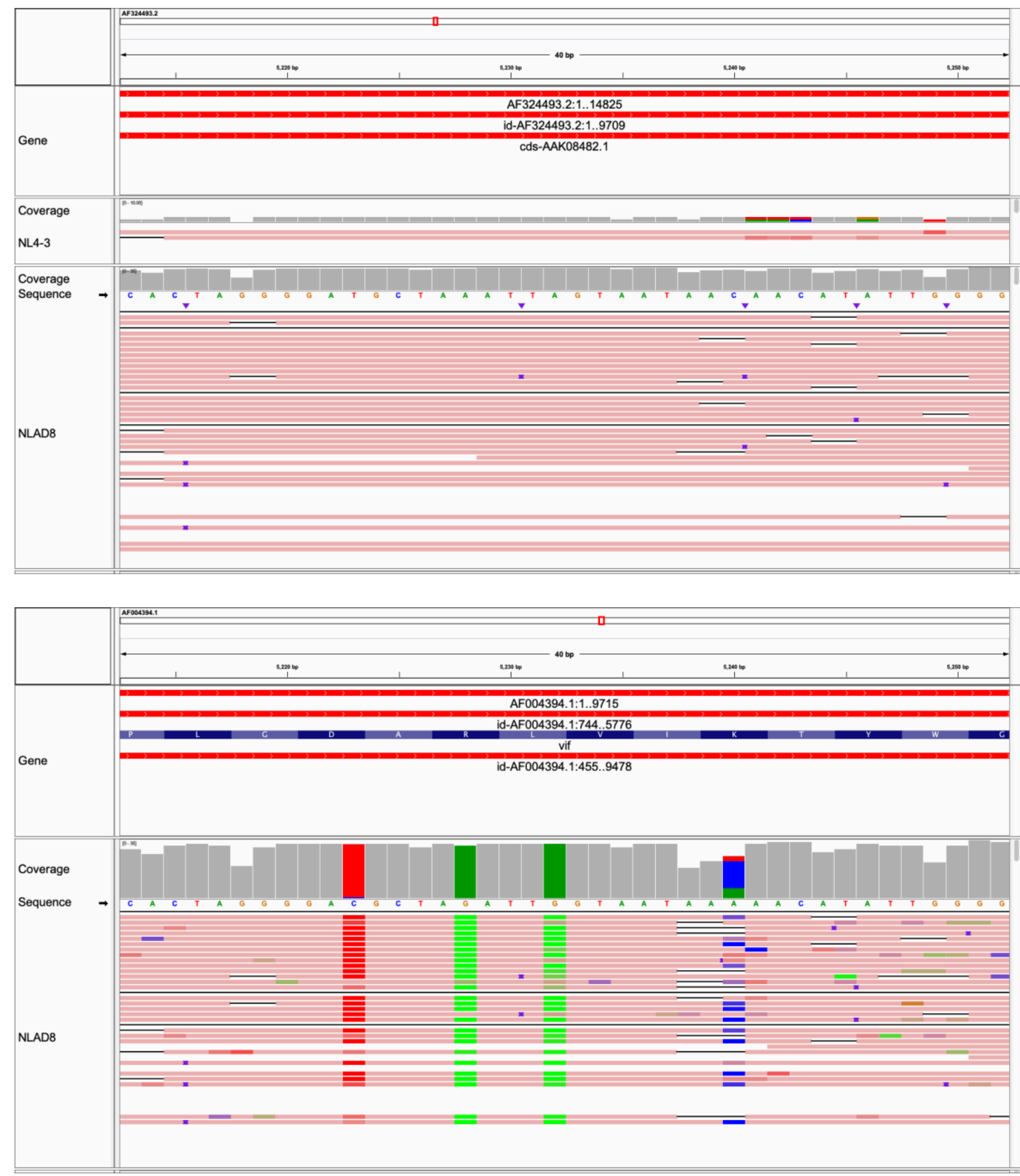

Top: AF324493.2:5,213-5,252. This region is common between NLAD8 and NL4-3 (top),

and we expect differences in parts of the virus outside the env gene (bottom). In above,

we see 4 SNVs, one embedded in a homopolymer track. The fact that we can read across

such a large A-homopolymer (thus more easily discounting the false positive deletions in

reads at the A-homopolymer) may either be due to a SNV breaking up the A- 
homopolymer, or less likely a shift in the relative position of C. Compare to SAME Region

in NL4-3. Bottom: AF004394.1:5,213-5,252. Note that the usage of SNVs here is to denote differences between reference samples NLAD8 and AD8, and not SNVs in NLAD8.

Alignments with minimap2 [13] in usegalaxy.eu [14]. Visualized in Integrative Genomics Viewer [49]. 
Supplemental Figure 4: Insertion
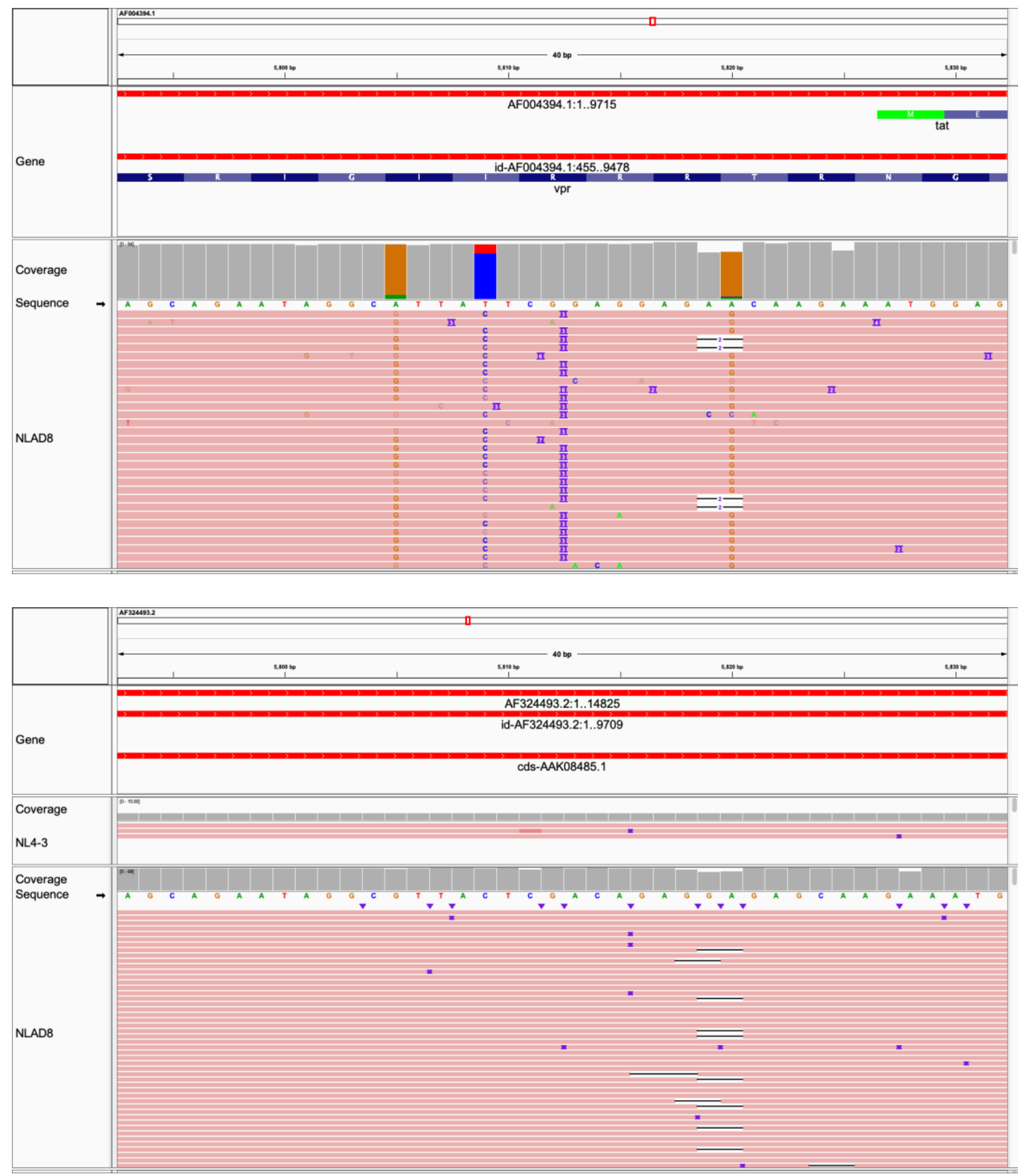

Top: AF004394.1:5,793-5,832. This region is common between NLAD8 and NL4-3, and we expect differences in parts of the virus outside the env gene. Compare to NL4-3. Bottom:

AF324493.2:5,793-5,832. Alignments with minimap2 [13] in usegalaxy.eu [14]. Visualized in Integrative Genomics Viewer [49]. 
Supplemental Figure 5: A likely true positive deletion with respect to reference AD8

in NLAD8
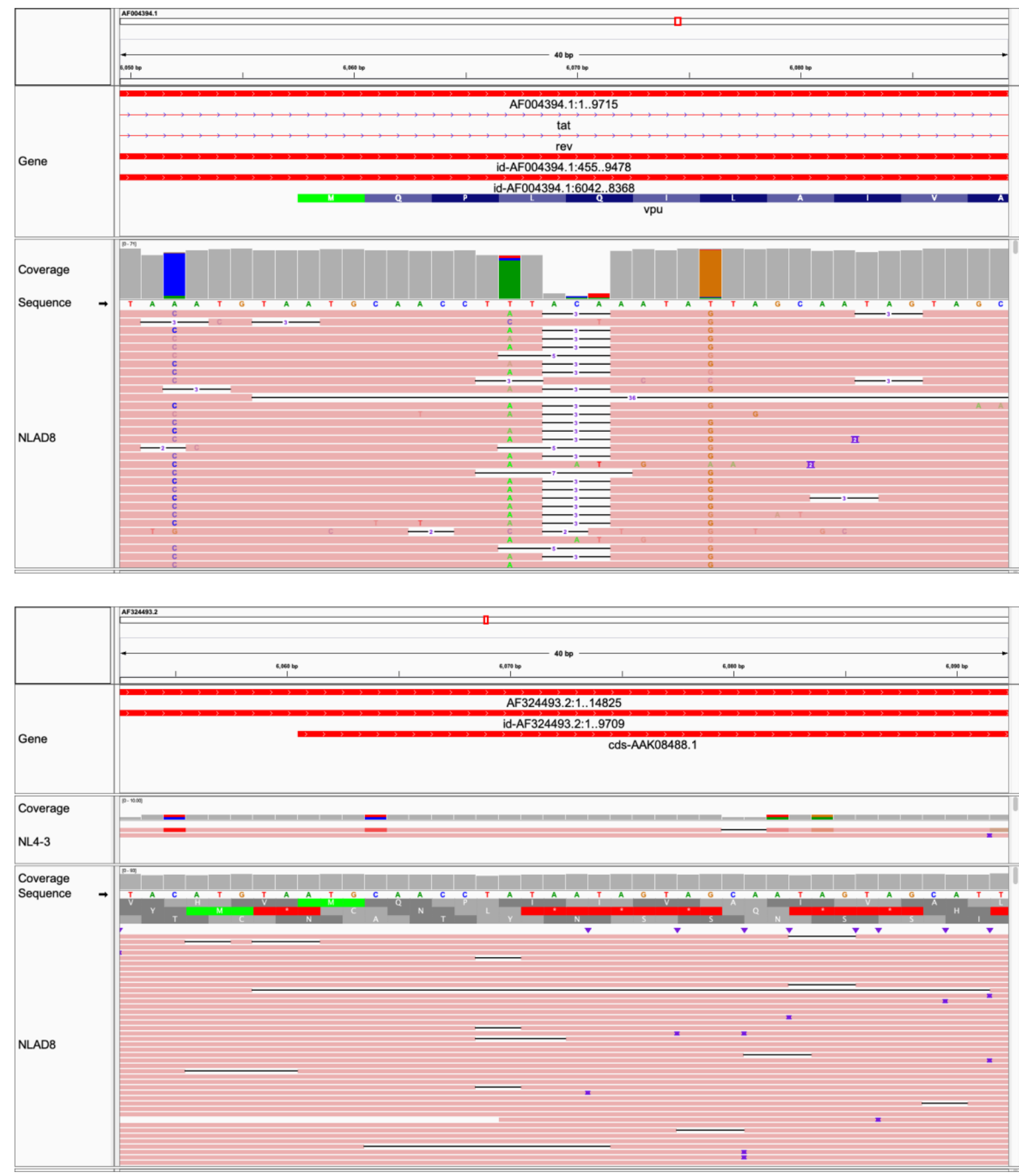

This region is common between NLAD8 and NL4-3, and we expect differences in parts of the virus outside the env gene. AF004394.1:6,050-6,089. While technically out of frame, given the context of the above, this deletion would cause an in-frame substitution of LQ 
bioRxiv preprint doi: https://doi.org/10.1101/845610; this version posted November 19, 2019. The copyright holder for this preprint (which was not certified by peer review) is the author/funder. All rights reserved. No reuse allowed without permission.

66

at amino acids 4 and 5 and gain of I at position 4 in vpu. Compare to NL4-3. Alignments

with minimap2 [13] in usegalaxy.eu [14].Visualized in Integrative Genomics Viewer [49]. 
Supplemental Figure 6: Structural variants intermingled with SNVS
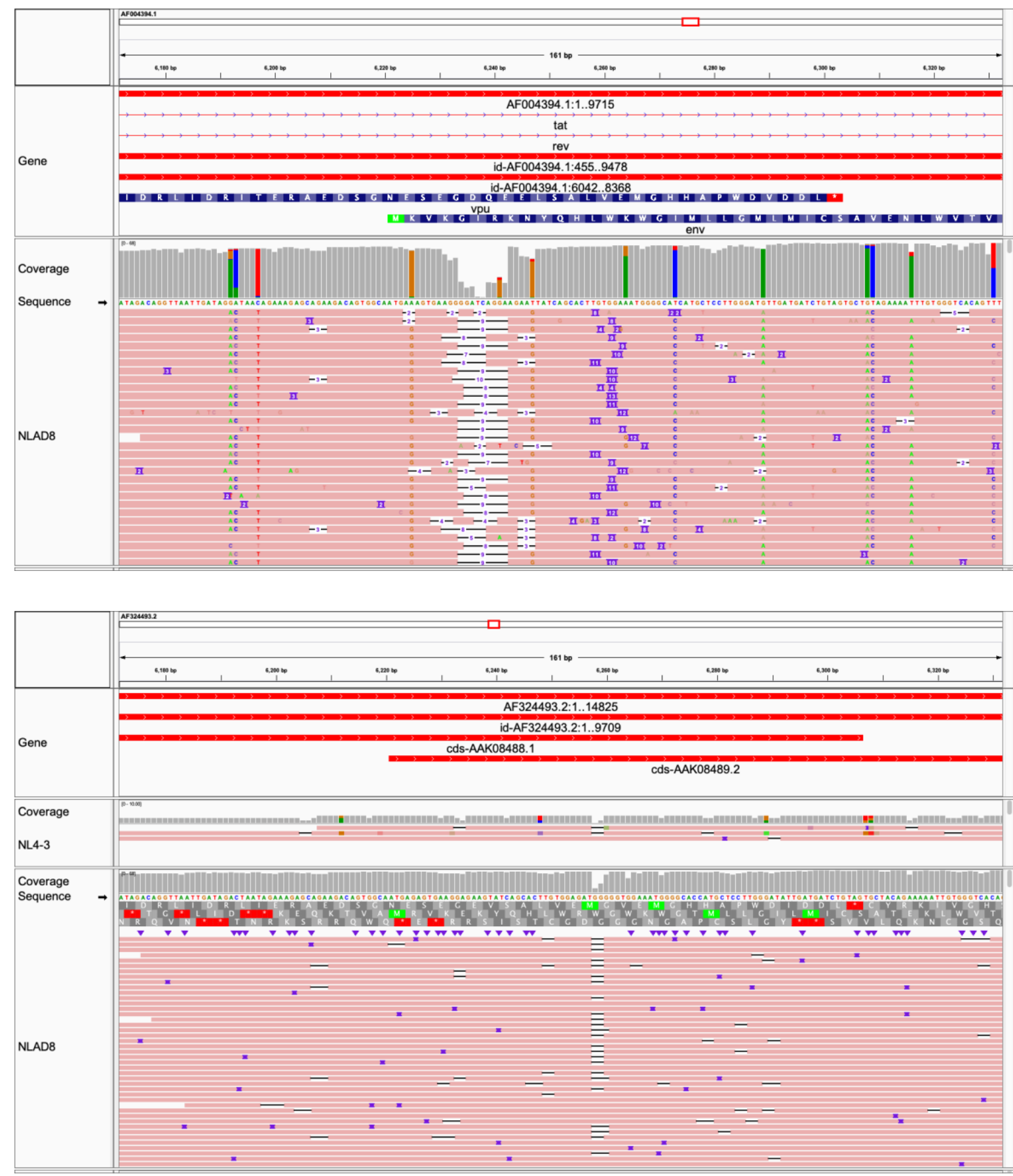

Top: AF004394.1:6,172-6,332. Bottom: AF324493.2:6,172-6,332. Region conserved

between NL4-3 and NLAD8 (bottom), but not between NLAD8 and AD8 (top). Alignments

with minimap2 [13] in usegalaxy.eu [14]. Visualized in Integrative Genomics Viewer [49]. 
Supplemental Figure 7: SNVs define HIV haplotypes directly.
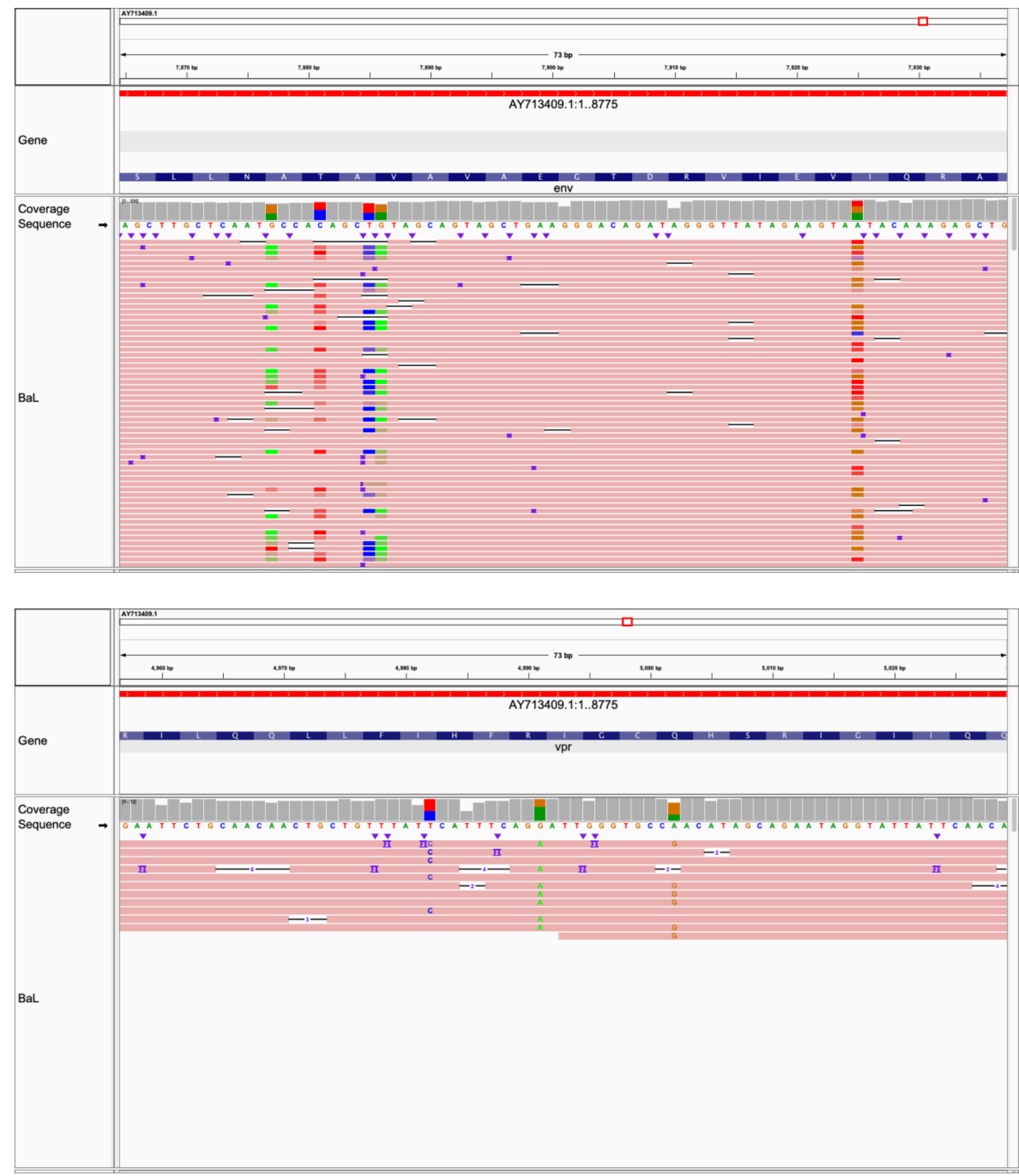

Haplotypes in BaL are observed to be mutually exclusive. Top: AY713409.1:7,865-7,937.

Bottom: AY713409.1:4,957-5,029. Note two haplotypes are seen here. One as g.4982T>C, another as g.4991G>A with g.5002A>G (other phased SNVs not mentioned). Alignments with minimap2 [13] in usegalaxy.eu [14]. Visualized in Integrative Genomics Viewer [49]. 
bioRxiv preprint doi: https://doi.org/10.1101/845610; this version posted November 19, 2019. The copyright holder for this preprint (which was not certified by peer review) is the author/funder. All rights reserved. No reuse allowed without permission.

69

Supplemental Table 3: SNVs in reference HIV-1 strains

NA: Nucleic Acid. AA: Amino acid. ASP: Antisense protein. LTR: Long terminal repeat. RT:

Reverse transcriptase. 
bioRxiv preprint doi: https://doi.org/10.1101/845610; this version posted November 19, 2019. The copyright holder for this preprint (which was not certified by peer review) is the author/funder. All rights reserved. No reuse allowed without permission.

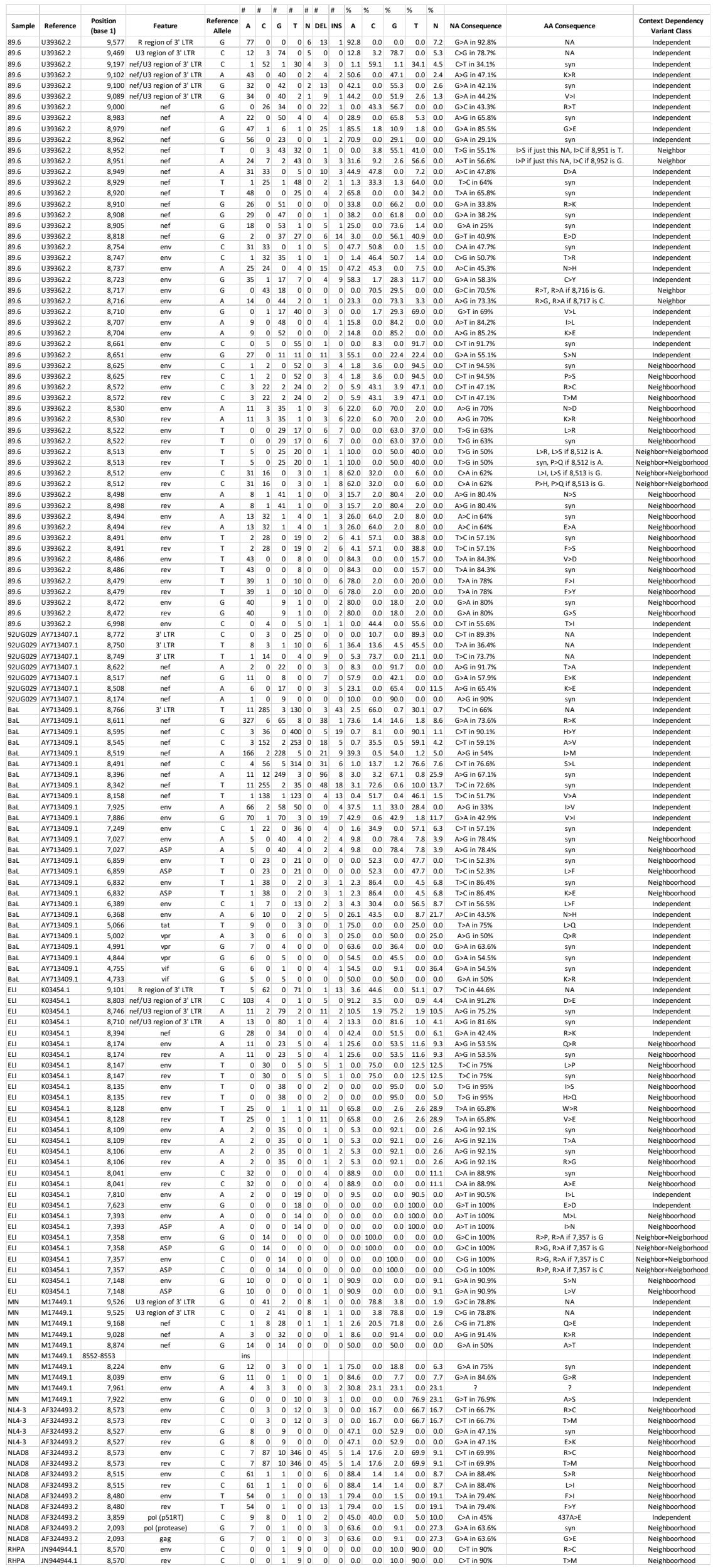


Supplemental figure 8: Context Dependency Variant Classification

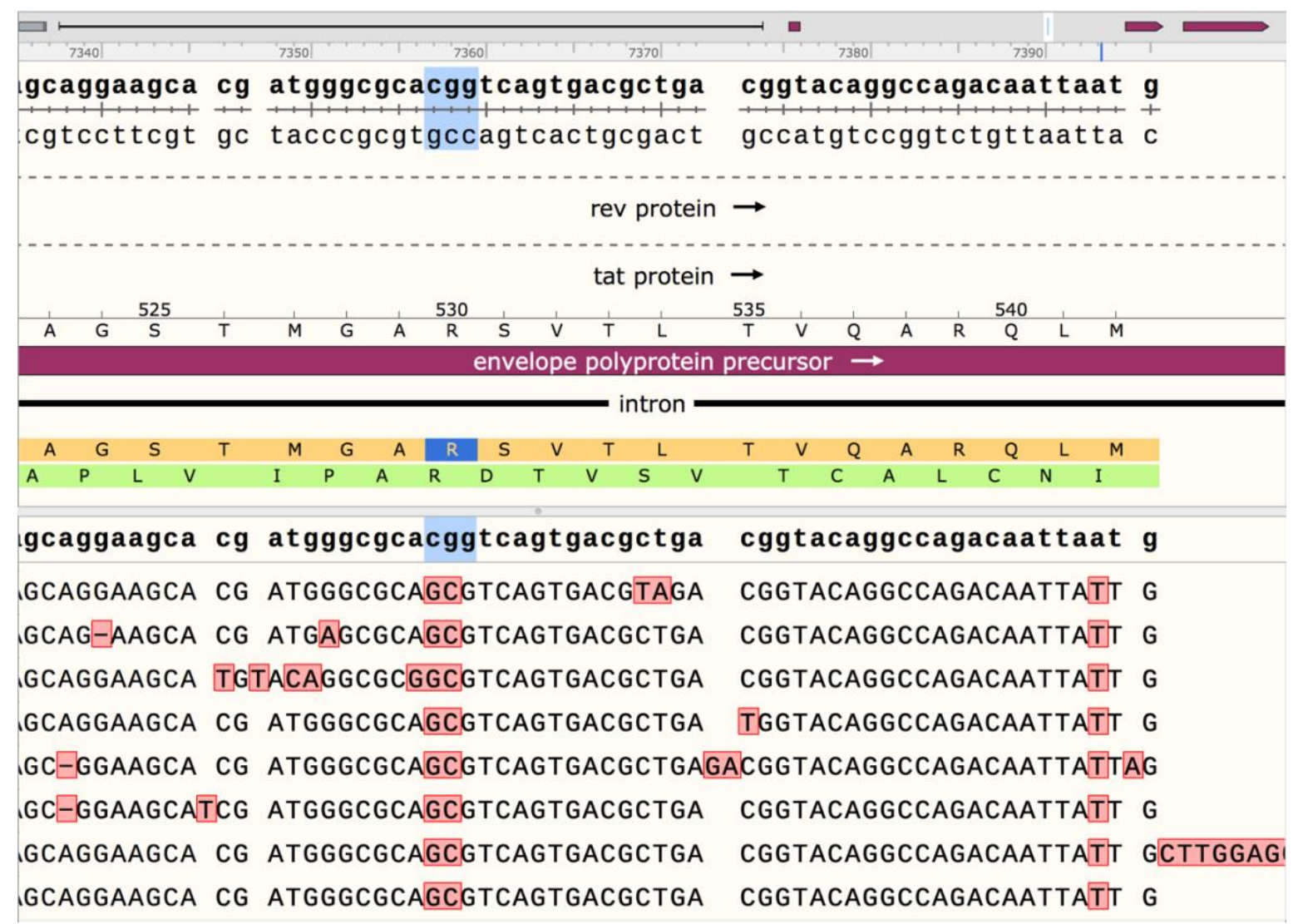

Independent $=$ a variant whose effect is limited to its coding frame. Neighbor = a variant

whose effect is limited to its coding frame, but when considering variation in its adjacent

nucleotide, can contribute to a codon change dependent on both states. Neighborhood =

a variant whose effect is not limited to its coding frame (example: when proteins are

coded by the same mRNA but in different frames as part of gagpol, tat/rev/env/vpu/ASP),

but when considering its adjacent nucleotide, can contribute to a pair of codons changing

dependent on both states. Neighbor+Neighborhood = when Neighbor and Neighborhood

dependencies are satisfied. Here we have a frame from ELI, where

Neighbor+Neighborhood occurs at R530 (highlighted in blue) in env (maroon and orange

in forward orientation), and also overlaps residues in Antisense protein (in green,

negative orientation). 
Supplemental Figure 9: Canu de novo assembly supports manual SNV calling

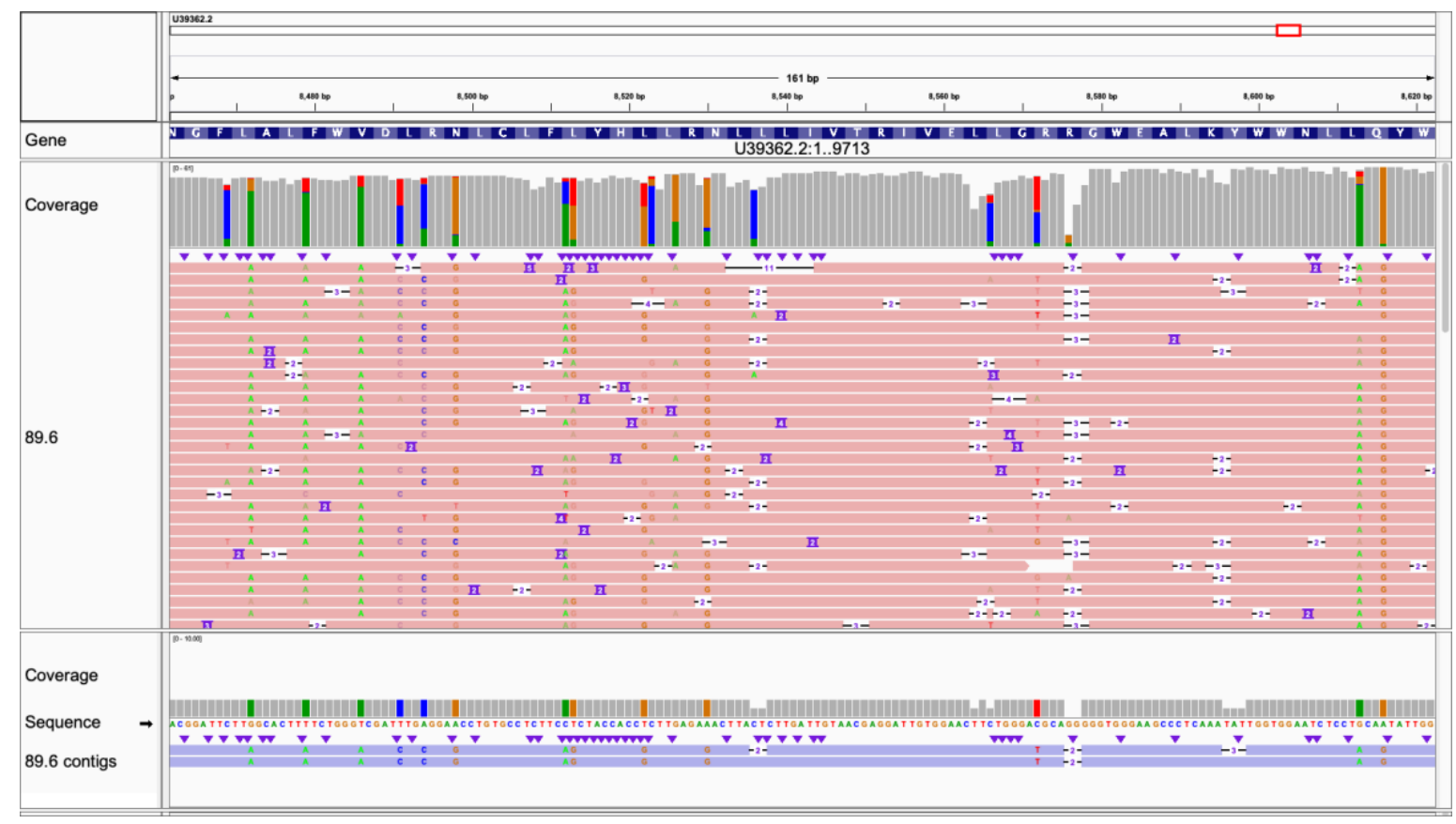

89.6. U39362.2:8,462-8,622. Note that contigs were assembled in negative orientation.

Alignments with minimap2 [13] in usegalaxy.eu [14]. Visualized in Integrative Genomics

Viewer [49]. 
Supplemental Figure 10: Canu de novo assembly can incompletely resolve haplotypes

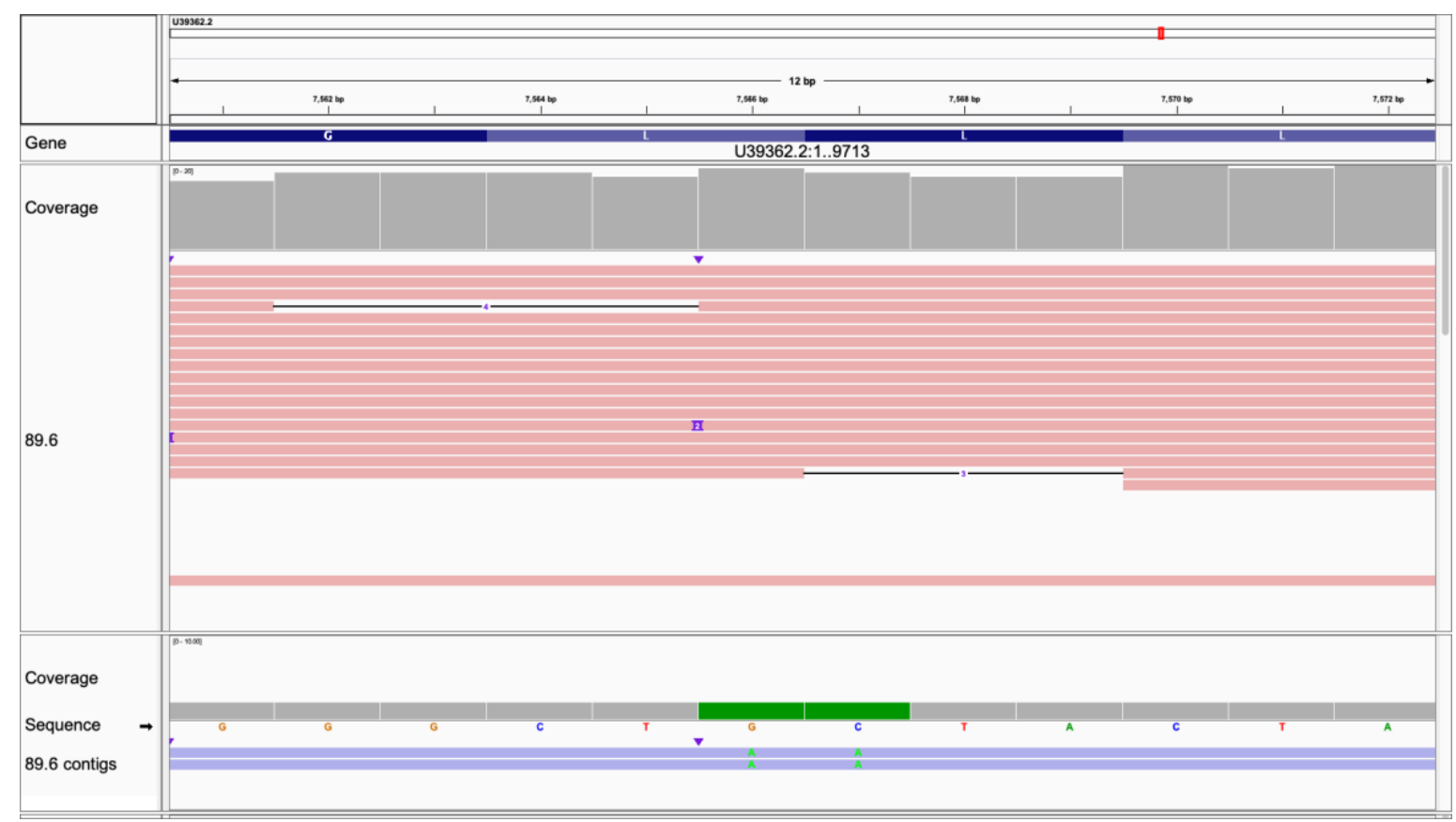

89.6. U39362.2:7,561-7,572. SNVs in assemblies not supported by visual inspection. Left

G: 4/14 A. Right C: 4/14 A. Note that contigs were assembled in negative orientation.

Alignments with minimap2 [13] in usegalaxy.eu [14]. Visualized in Integrative Genomics

Viewer [49]. 
Supplemental Figure 11: De novo contigs can provide drug resistance information
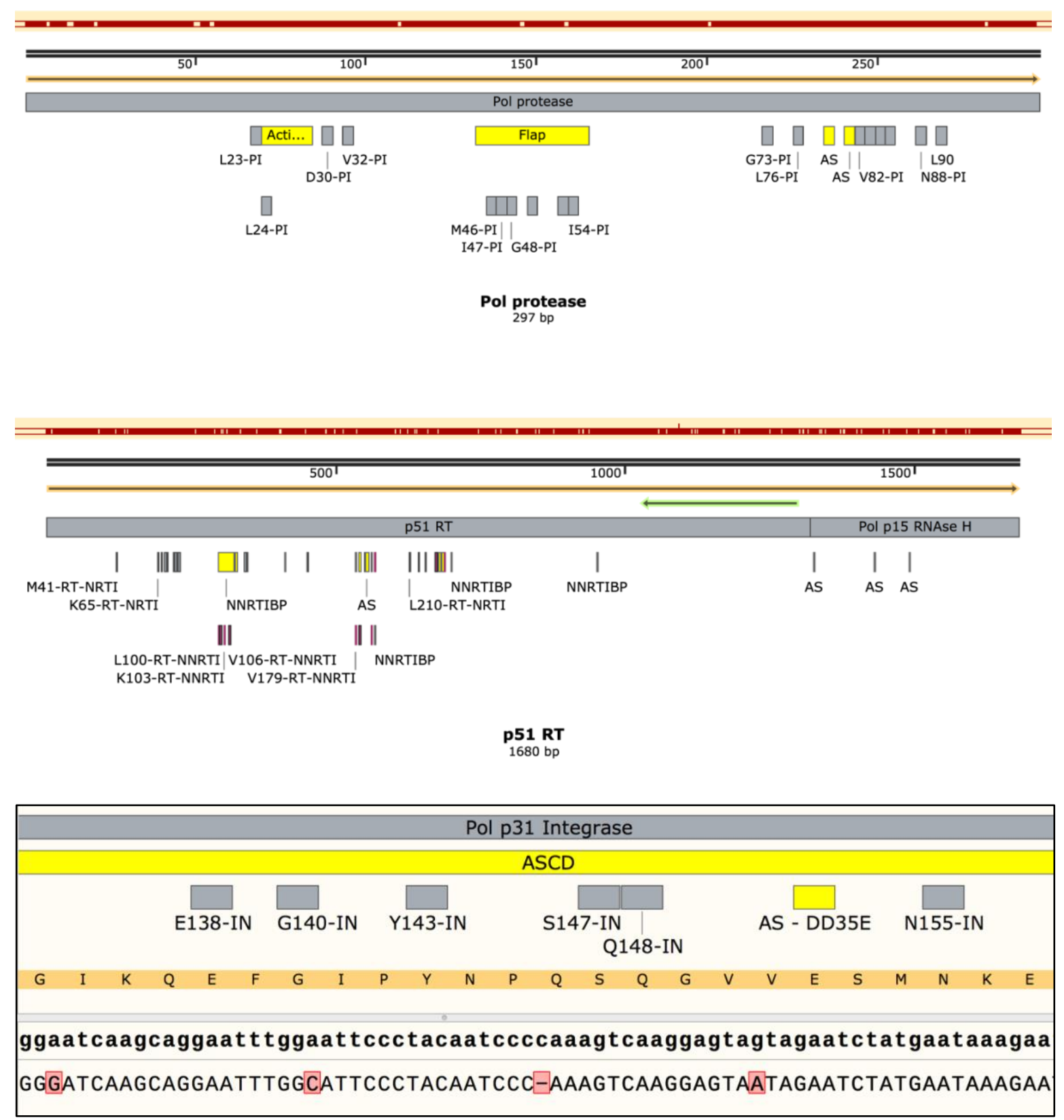

Drug resistance data from Stanford HIV Drug Resistance Database [23,24]. Reference

HXB2 K03455.1. Maroon line is the mapped NLAD8 contig. Close-up of integrase shows

silent mutation at G140G and a missense mutation at V151I, both around known

resistance-associated residues. The truncated $\mathrm{C}$ homopolymer in the center of the

viewing window is most likely an ONT artifact. Visualized in SnapGene version 5.0.4. 
Supplemental Figure 12: Short-read NL4-3 virion RNA-seq
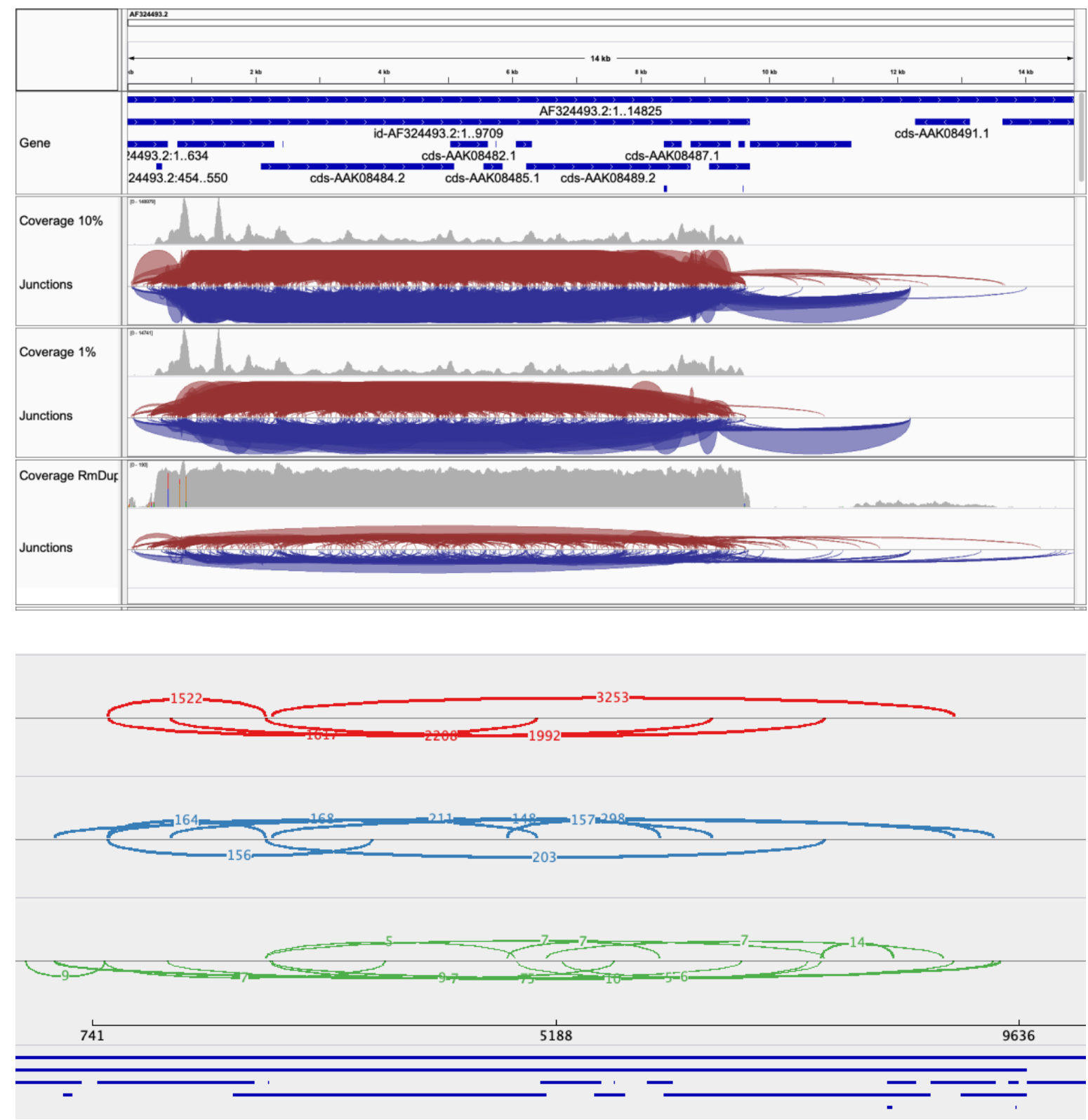

Single-end 76 bp. SRR3472915. Reference: pNL4-3 AF324493.2. Note backbone

contamination, either due to backbone DNA contamination or backbone insertion and

expression. Forensic genomic analyses support cDNA + PCR library, although this

information was not available from SRA entry. Top: mapped with HISAT2 (a split-read

aligner/mapper [50]). Note abundance of forward and reverse splice junctions. Bottom:

Sashimi plot of forward splice junctions only. Red: data downsampled to $10 \%$. Minimum

splice junction frequency set to 1470 . Blue: data downsampled to $1 \%$. Minimum splice 
bioRxiv preprint doi: https://doi.org/10.1101/845610; this version posted November 19, 2019. The copyright holder for this preprint (which was not certified by peer review) is the author/funder. All rights reserved. No reuse allowed without permission.

junction frequency set to 147 . Green: data processed with RmDup to remove PCR duplicates. Minimum splice junction frequency set to 5 . Most reads include 3'LTR elements, and a canonical exon upstream of gag. 
bioRxiv preprint doi: https://doi.org/10.1101/845610; this version posted November 19, 2019. The copyright holder for this preprint (which was not certified by peer review) is the author/funder. All rights reserved. No reuse allowed without permission.

LIST OF DATA (To be deposited in SRA or ENA in near future)

Basecalled files

.dna files of contigs ( $n=$ ?)

AF033819.3-anchored .bam/.bai files

Strain-specific .bam/.bai files

Any multi-sequence alignments 